International Journal of Child, Youth and Family Studies (2014) 5(4): 493-587

In Harm's Way: A Special Issue on the Impacts and Costs of Witnessing Intimate Partner

Violence

\title{
A COMPREHENSIVE REVIEW OF THE LITERATURE ON THE IMPACT OF EXPOSURE TO INTIMATE PARTNER VIOLENCE FOR CHILDREN AND YOUTH
}

\section{Sibylle Artz, Margaret A. Jackson, Katherine R. Rossiter, Alicia Nijdam-Jones, István Géczy, and Sheila Porteous}

\begin{abstract}
Children living in homes where intimate partner violence occurs are often exposed to such violence through witnessing, seeing its effects, hearing about it, or otherwise being made aware that violence is taking place between parents or caregivers. Exposure to intimate partner violence is considered to be a form of child maltreatment, and affected children are often also the victims of targeted child abuse. This paper presents findings from a comprehensive review of the literature on the impact of exposure to intimate partner violence for children and youth, focusing on: (a) neurological disorders; (b) physical health outcomes; (c) mental health challenges; (d) conduct and behavioural problems; (e) delinquency, crime, and victimization; and (f) academic and employment outcomes. The notion of cascading effects informed our framework and analysis as it became evident that the individual categories of impacts were not only closely related to one another, but in a dynamic fashion also influence each other in multiple and interconnected ways over time. The research reviewed clearly shows that children who are exposed to intimate partner violence are at significant risk for lifelong negative outcomes, and the consequences are felt widely in society.
\end{abstract}

Keywords: children, domestic violence, exposure, intimate partner violence, witnessing, youth

Acknowledgements: This project was supported by the Social Sciences and Humanities Research Council. Portions of this article were presented at the 2012 National Research Day Conference in Vancouver, Canada. 
International Journal of Child, Youth and Family Studies (2014) 5(4): 493-587

In Harm's Way: A Special Issue on the Impacts and Costs of Witnessing Intimate Partner Violence

Sibylle Artz, Ph.D. (the corresponding author) is a Professor in the School of Child and Youth Care at the University of Victoria, P.O. Box 1700, STN CSC, Victoria, British Columbia, Canada, V8W 2Y2. E-mail: sartz@uvic.ca

Margaret A. Jackson, Ph.D. is a Professor Emerita in the School of Criminology at Simon Fraser University and Director at the FREDA Centre for Research on Violence Against Women and Children, 515 West Hastings Street, Vancouver, British Columbia, Canada, V6B 5K3. Email: margarej@sfu.ca

Katherine R. Rossiter, Ph.D. is an Adjunct Professor in the School of Criminology at Simon Fraser University, and Postdoctoral Fellow and Associate Director at the FREDA Centre for Research on Violence Against Women and Children, 515 West Hastings Street, Vancouver, British Columbia, Canada, V6B 5K3. E-mail: rossiter@sfu.ca

Alicia Nijdam-Jones, M.A. is a Research Associate at the FREDA Centre for Research on Violence Against Women and Children, 515 West Hastings Street, Vancouver, British Columbia, Canada, V6B 5K3. E-mail: ahn@sfu.ca

István Géczy, Ph.D. is a Psychology Instructor at Northern Lights College, 9820 - 120th Avenue, Fort St. John, British Columbia, Canada, V1J 6K1. E-mail: igeczy@nlc.bc.ca

Sheila Porteous, B.A. is a Child, Youth, and Family Counsellor at Beacon Community Services and a Master's Student in the School of Child and Youth Care at the University of Victoria, P.O. Box 1700, STN CSC, Victoria, British Columbia, Canada, V8W 2Y2. E-mail: sheilap2@uvic.ca 
International Journal of Child, Youth and Family Studies (2014) 5(4): 493-587

In Harm's Way: A Special Issue on the Impacts and Costs of Witnessing Intimate Partner

Violence

\section{Table of Contents}

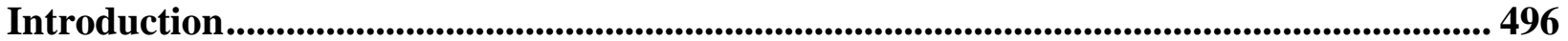

Family Violence and Child Maltreatment in Canada ......................................................................4496

Research on Children's Exposure to Intimate Partner Violence ................................................... 497

Need for a Comprehensive Review................................................................................................................ 499

Literature Review Methodology........................................................................................................501

Neurological Disorders ...................................................................................................... 502

Neurodevelopmental Consequences of Childhood Maltreatment............................................................504

Neurobiological Regulation in Infants Exposed to IPV ........................................................................505

Physical Health Outcomes............................................................................................ 512

Health Conditions and Use of Health Services .............................................................................................. 514

Nutritional Status and Mortality .................................................................................................................. 516

Mental Health Challenges: Internalizing and Externalizing Issues................................... 517

Mediators in the Relationship Between IPV Exposure and Behaviour Problems ........................... 521

Attention Problems .............................................................................................................................................. 524

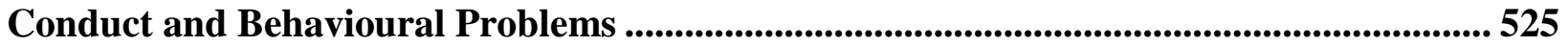

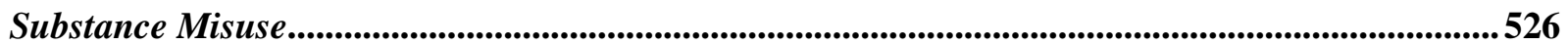

Delinquency, Crime, and Victimization................................................................................... 537

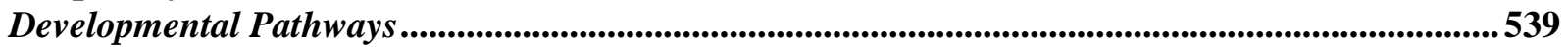

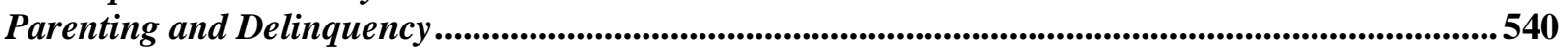

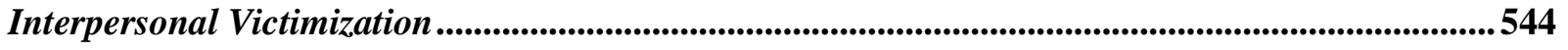

Academic and Employment Outcomes ....................................................................................... 545

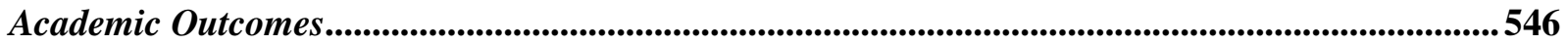

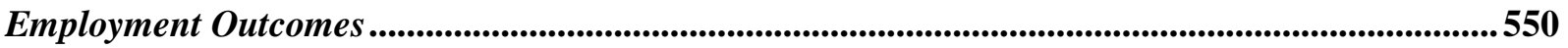

Conclusions: The Weight of the Evidence ................................................................................. 552

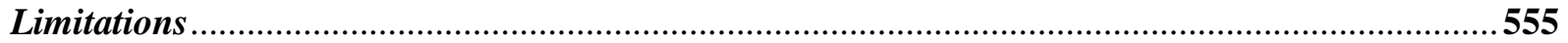

References .................................................................................................................................. 558 
International Journal of Child, Youth and Family Studies (2014) 5(4): 493-587

In Harm's Way: A Special Issue on the Impacts and Costs of Witnessing Intimate Partner

Violence

\section{Introduction}

In the early 1980s, the Parliament of Canada had established an all-party committee to address the problem of domestic violence and shelters. On May 12, 1982, Margaret Mitchell, NDP Member of Parliament [MP] for Vancouver East, rose in the House of Commons to ask the minister responsible for the Status of Women why there were not more shelters for battered women. She began by noting that the parliamentary report tabled in the House the day before stated that one in 10 women was beaten by their husband. The mostly male House did not take the statement seriously and a ripple of laughter was heard from all sides of the House. One MP declared, “I don't beat my wife”. Women across the country were angry, prompting an apology from the House to Canadian women (see House of Commons Debates, 1982, p. 17334).

\section{Family Violence and Child Maltreatment in Canada}

In Canada, as in every country in the world, violence that is committed against family members is seen as a serious and pervasive issue, although this has not always been the case. As recorded in the latest report on family violence released by Statistics Canada (Sinha, 2013), the total number of police-reported family violence crimes (including dating violence) in 2011 was 94,839 , a number that constitutes $25.5 \%$ of all violent crime for that year. More than $69 \%$ of the victims of family violence crimes were female $(65,587)$ rather than male $(29,252)$. Despite the ominous and inescapable presence of family violence in everyday life, it has only been within the past 30 years that the perception of violence, aggression, and threats against family members has changed from being considered a private matter within the family to being recognized as a serious crime (Sinha, 2013).

The link between the experience of abuse by family members, mostly parents, and negative outcomes for children and youth has been under discussion at least since Sheldon and Eleanor Glueck (1950) published their seminal work on the subject in the decade after the end of the Second World War. However, the connection between the abuse of children and youth, the general level of violence in a family, and the perpetration of violence upon intimate partners, most often mothers, was not seen as a core issue until the 1980s. In the aftermath of the second wave of feminism and growing public awareness of the plight of abused women and their children, attention turned specifically to the effects on children of exposure to intimate partner violence (IPV) (Bedi \& Goddard, 2007; Carlson, 1984). As Bedi and Goddard (2007) note, "although it has long been recognized that different forms of violence may co-exist [...] research addressing the overlap between IPV and child abuse is a relatively recent phenomenon” (p. 67). Bedi and Goddard reviewed 117 research articles on the co-occurrence of IPV and targeted child abuse, examined the possible reasons for the overlap, and summarized the impacts on children of living with IPV and child abuse. They conclude that the "substantial overlap between families in which targeted child abuse occurs and those characterized by IPV has resulted in a population of doubly traumatised children” (p. 72). Based on their findings, they suggest that exposure to IPV is itself a form of child abuse - a position already enshrined in child protection policy in New South Wales, Australia. 
International Journal of Child, Youth and Family Studies (2014) 5(4): 493-587

In Harm's Way: A Special Issue on the Impacts and Costs of Witnessing Intimate Partner Violence

Children living in homes with domestic violence are often exposed to this violence, through witnessing physical violence between parents or caregivers, seeing the effects of violence (e.g., injuries, broken furniture), hearing about violence, or otherwise being made aware of this violence. Children's exposure to IPV also includes "being used as a tool of the perpetrator" (Olofsson, Lindqvist, Gådin, Bråbäck, \& Danielsson, 2011, p. 89). Research tells us that families experiencing domestic violence are more likely than non-violent families to have children present (Bedi \& Goddard, 2007). Young children are especially likely to bear witness to violence in the home, and also to experience abuse and neglect themselves - two problems that often cooccur and may contribute to negative outcomes for children, in a range of arenas (Carpenter \& Stacks, 2009; Herrenkohl, Sousa, Tajima, Herrenkohl, \& Moylan, 2008). It is increasingly recognized that witnessing family violence is as harmful as experiencing it directly. Often parents believe that they have shielded their children from spousal violence, but research shows that children see or hear some $40 \%$ to $80 \%$ of it and that these children suffer the same consequences as those who are abused directly (Royal Canadian Mounted Police, 2012). Saltzman, Holden, and Holahan (2005) even drew the conclusion that the "psychological scars" borne by children who are exposed to violent interactions between their parents could be more detrimental than those of children who had been the direct targets of physical abuse by a parent.

\section{Research on Children's Exposure to Intimate Partner Violence}

Research on children's exposure to IPV has grown significantly over the past 30 years, though it is still less developed than research on other problems such as child abuse and maltreatment (Wolfe, Crooks, Lee, McIntyre-Smith, \& Jaffe, 2003). The first case studies of the effects of domestic violence exposure on children emerged in the late 1970s, with the initial empirical studies published in the early 1980s. What is referred to as the "first wave" of research in this area has been criticized for its serious methodological limitations, while the "second wave" of research - literature published since the early 1990s - is considered to be much more sophisticated (Evans, Davies, \& DiLillo, 2008). A number of early reviews were published on the effects of children's exposure to domestic violence (e.g., Fantuzzo \& Lindquist, 1989; Kolbo, Blakely, \& Engleman, 1996), with several more published since the year 2000 generally reporting on research from the 1990s or later, though a few include literature dating back as far as the late 1960s and 1970s.

It is clear from the available research that IPV can have an enduring adverse effect on diverse domains of children's development, even if children are only indirectly exposed to domestic conflicts, for example, by observing the incident, experiencing the aftermath, or hearing about the event (Holden, 2003). The pervasive influence of the problem is illustrated by the fact that in the United States alone an estimated 10 million children are exposed to IPV each year, and children under 6 years of age are more likely to be affected than older children (Carpenter \& Stacks, 2009). Data collected on 7,865 children and their families by the British Office for National Statistics found that 4.3\% $(n=340)$ of these children had witnessed severe incidents of domestic violence, and that witnessing IPV was the third most frequently reported trauma for children (Meltzer, Doos, Vostanis, Ford, \& Goodman, 2009). Of the children who witnessed severe domestic violence, 30\% also experienced another traumatic event such as 
International Journal of Child, Youth and Family Studies (2014) 5(4): 493-587

In Harm's Way: A Special Issue on the Impacts and Costs of Witnessing Intimate Partner Violence

witnessing violence against their family members or friends, or experiencing a serious and frightening accident (Meltzer et al., 2009).

International data suggest an undeniable link between childhood exposure to IPV and adverse life outcomes (Ackerson \& Subramanian, 2008; Roustit et al., 2009). In their retrospective cohort study of 3,023 adult participants in Paris, Roustit et al. (2009) found that individuals who witnessed domestic violence during childhood were $44 \%$ and $75 \%$ more likely to develop symptoms of depression and alcohol dependence, respectively. Roustit et al. (2009) also observed, moreover, that the association between childhood exposure to IPV and domestic violence perpetration is the strongest. The presence of IPV elevated the risk of perpetrating IPV and child abuse by more than three times and almost five times, respectively.

Child maltreatment in Canada has been acknowledged as a public health concern (Afifi, 2011). For instance, the Canadian Incidence Study of Reported Child Abuse and Neglect (CIS report), a comprehensive nationwide study conducted in 2008 on the physical and psychological health of Canadian children, found that almost 150,000 children were either maltreated or were at risk of future maltreatment (Public Health Agency of Canada, 2010). The CIS report also revealed that exposure to IPV appears to be the most common form of abuse affecting nearly 30,000 children of the 85,000 cases where investigations revealed substantiated maltreatment (categories included sexual abuse, neglect, emotional maltreatment, and exposure to IPV). Intervention from Child Welfare Services, on the other hand, is less likely to occur when the child is affected by IPV compared to other forms of maltreatment (Black, Trocmé, Fallon, \& MacLaurin, 2008). Researchers have hence concluded that the "Canadian child welfare system is substantiating exposure to domestic violence but is concluding that these families do not require ongoing child welfare services” (Black et al., 2008, p. 403).

This position, however, can be contrasted with converging new evidence that early-onset exposure to domestic violence is associated with maladaptive courses of child development culminating in a less optimal outlook through the entire lifespan (Carpenter \& Stacks, 2009; Yount, DiGirolamo, \& Ramakrishnan, 2011). In much the same vein, the CIS report concluded that an abusive family environment is linked to a high incidence of adjustment problems among Canadian children of all ages in the domains of social conduct, intellectual/academic performance, mental health (i.e., anxiety, hyperactivity), and attachment (Public Health Agency of Canada, 2010). Corroborating data from a meta-analysis of 60 related studies published between 1990 and 2006 - drawing mainly on samples from locations in the United States - also indicate that mental health and behavioural problems in children (i.e., internalizing and externalizing behaviours) are moderately associated with violence exposure at home (Evans et al., 2008).

Finally, Wood and Sommers (2011) have argued that the severity of symptoms may worsen if children are exposed to multiple types of family violence, also often referred to as the dual exposure or “double whammy" effect (Moylan et al., 2010). Exposure to maltreatment may endanger children's physical and psychosocial development, particularly if families have insufficient support (Noll \& Shenk, 2010). Although child abuse is thought to be the most severe form of maltreatment, exposure to ongoing disputes and adversities between parents or 
International Journal of Child, Youth and Family Studies (2014) 5(4): 493-587

In Harm's Way: A Special Issue on the Impacts and Costs of Witnessing Intimate Partner Violence

caregivers in the home environment, such as IPV, are known to have severe detrimental effects on the developing infant (for recent reviews of the literature see Adams, 2006; Buckley, Holt, \& Whelan, 2007; Carpenter \& Stacks, 2009; Evans et al., 2008; Holt, Buckley, \& Whelan, 2008; Howell, 2011; Wood \& Sommers, 2011; Yount et al., 2011).

To summarize the main points of these arguments, research emanating from the last two decades attests that children are often exposed to different forms of domestic violence simultaneously (i.e., directly experiencing as well as witnessing abuse, see Margolin \& Vickerman, 2011; Moylan et al., 2010), yet exposure to IPV has an independent contribution to symptoms of maladaptive child development (Carpenter \& Stacks, 2009; Yount et al., 2011). The majority of the review articles published recently focus on the effects of children's exposure to intimate partner or inter-parental violence and aggression, while some have a broader definition of "family violence" that includes other types of violence, such as child abuse, sibling violence, and even violence towards pets (e.g., Martin, 2002). Other reviews include both exposure to inter-parental violence and direct victimization (e.g., Bedi \& Goddard, 2007; Chan \& Yeung, 2009; Herrenkohl et al., 2008; Sternberg, Baradaran, Abbott, Lamb, \& Guterman, 2006), in an effort to compare groups of children who are exposed to violence, those experiencing it directly, and those who both witness and experience violence themselves.

Research in this area has focused on a range of child outcomes including the following: delays in neurological development (Carpenter \& Stacks, 2009); health outcomes and use of health services (Bair-Merritt, Blackstone, \& Feudtner, 2006; Wood \& Sommers, 2011); internalizing and externalizing problems or behaviours (Bedi \& Goddard, 2007; Evans et al., 2008; Martin, 2002; Sternberg, Baradaran, et al., 2006; Wolfe et al., 2003); trauma symptoms or PTSD (Bedi \& Goddard, 2007; Evans et al., 2008); adjustment and developmental problems (Chan \& Yeung, 2009; Herrenkohl et al., 2008; Holt et al., 2008; Rhoades, 2008); and academic and social outcomes (Fowler \& Chanmugam, 2007). It is clear from the literature that both targeted child abuse and exposure to IPV contribute substantively to an array of problematic outcomes for children and youth. However, not all children experience adverse effects, and it remains unclear which children are at the greatest risk for which negative outcomes, or not at risk despite their exposure to domestic violence in all its forms.

\section{Need for a Comprehensive Review}

As society can incur significant costs from both child abuse and maltreatment, prevention efforts should begin with a basic understanding of the factors which mediate or moderate the effect of early-onset exposure to domestic violence (Mohr \& Fantuzzo, 2000; Noll \& Shenk, 2010). Until quite recently, however, in mainstream research "pathways between marital conflict and children's development have typically been illuminated by means of a mediating effect of parenting [...] few have explored direct links between marital discord and emerging physiological and emotional regulation indexes in infancy” (Porter, Wouden-Miller, Silva, \& Porter, 2003, p. 298). Careful inspection of six comprehensive papers from the field (reviews and meta-analyses) published since 2008 shows, indeed, that neurodevelopmental implications of witnessing domestic violence are addressed, to a certain extent, in only two of these articles (Carpenter \& Stacks, 2009; Yount et al., 2011) but ignored in the rest (Evans et al., 2008; Holt et 
International Journal of Child, Youth and Family Studies (2014) 5(4): 493-587 In Harm's Way: A Special Issue on the Impacts and Costs of Witnessing Intimate Partner Violence

al., 2008; Howell, 2011; Wood \& Sommers, 2011). Although both neuroscientists and social scientists consider human development a focal point in their research interest (Nelson \& Bloom, 1997), cross-fertilization between the two disciplines has just recently begun to facilitate a broader understanding of how “children's competencies develop across multiple domains and progress along a trajectory of critical stages in a web of complex transactions among environmental and ontogenic characteristics” (Mohr \& Fantuzzo, 2000, p. 71).

In a comprehensive review of the psychobiological literature on childhood maltreatment, published between 1990 and 2007, Grassi-Oliveira, Ashy, and Stein (2008) found strong support for a direct link between children's exposure to abuse or neglect and neurodevelopmental abnormalities in brain structures implicated in memory formation (hippocampus), emotional regulation (amygdala), inter-hemispheric communication (corpus callosum), and executive functions (prefrontal cortex). Furthermore, a series of carefully designed neuroimaging and endocrinological studies on infants with institutionalized backgrounds, representing the most extreme form of social and emotional deprivation, has found evidence of: (a) diminished white matter connectivity in higher cortical areas controlling emotional impulses; (b) lowered levels of hormones (oxytocin and vasopressin) linked to affiliative or positive social behaviours; as well as (c) dysregulation of the hypothalamic-pituitary-adrenal axis functions that mediate a complex array of physiological responses related to stress reactivity such as hormone production (e.g., glucocorticoid, Denver \& Crespi, 2006) and emotionality (Bos et al., 2011). Finally, findings from the Adverse Childhood Experiences (ACE) study ${ }^{1}$ indicate a dose-response relationship between severity of abusive childhood experiences and the number of comorbid outcomes in adult life (Anda et al., 2006). Specifically, individuals reporting the greatest number of childhood adversities at the baseline survey (i.e., those with the highest ACE scores) had, on average, three times more comorbid health-related conditions as adults than those with few adverse childhood experiences (i.e., those with the lowest ACE scores). By contrast, those who obtained a moderate ACE score suffered twice as many comorbid health outcomes as those who were not exposed to adversity in the home as a child (Anda et al., 2006).

Collectively, these studies illustrate clearly that chronic exposure to multiple sources of childhood maltreatment would be related to an accumulated effect on the individual with lifelong consequences that often manifest years after the exposure. In addition, a major implication of the ACE study is the proposed convergence of evidence from epidemiological and neurobiological data. For example, dysfunctional short-term memory (STM) processes observed in adults who were regularly exposed to abuse (Bremner et al., 1995) or severe social deprivation (Bos, Fox, Zeanah, \& Nelson, 2009) during infancy could be related to reduced volume in their brain structures (hippocampus) (McCrory, De Brito, \& Viding, 2011). Similarly, evidence on

\footnotetext{
${ }^{1}$ The ACE study (see Anda et al., 2006) is a large scale population-based epidemiological study in the United States $(N=17,337)$ to assess the relationship between childhood exposure to maltreatment and its effect in adulthood, measured in terms of indicators of physical and mental illness and high-risk behaviours. The study combines retrospective reports of adverse childhood experiences at baseline and prospective follow-up of the study cohort on diverse health outcomes that include incidence of diseases, health care utilization, premature mortality and causes of death. ACE scores are composite values calculated by summing the five different forms of ACEs to which the person recalled being exposed in the household as a child (alcohol or other substances, mental illness, violent treatment of mother or stepmother, criminal behaviour, and parental separation). Current health outcomes of the respondents included categories of mental health and somatic disturbances as well as substance abuse and sexuality.
} 
International Journal of Child, Youth and Family Studies (2014) 5(4): 493-587

In Harm's Way: A Special Issue on the Impacts and Costs of Witnessing Intimate Partner Violence

heightened risk for externalizing behaviours such as attention deficit/hyperactivity disorder, oppositional defiant disorder, and conduct disorder (Bos et al., 2011), or elevated aggression (Evans et al., 2008) in individuals exposed to adversities during their infancy, is consistent with neuroimaging and neuropsychological studies demonstrating a marked reduction in the size of the prefrontal cortex (i.e., the most advanced cortical structure mediating executive functions and controlling emotions) in individuals with a history of childhood adversity (McCrory et al., 2011).

Such shared findings might have the "potential to unify and improve our understanding of many seemingly unrelated, but often co-morbid health and social problems that have historically been seen and treated as categorically independent in the Western culture" (Anda et al., 2006, p. 181). This perspective is within the developmental ecological approach (Mohr \& Fantuzzo, 2000), which views a particular trajectory of child development as an "emerging property" (i.e., a unique outcome of life perspective) and as a web of complex interactions between individual factors and environmental characteristics (Hertzman, 2012). The developmental ecological model allows for accounts of lifelong phenotypic variations postulating a subtle interplay between early childhood experiences and the person's neurobiological variables. For example, health conditions common in adulthood (e.g., diabetes, obesity, hypertension, and cardiovascular diseases) have typically been assumed to result from lifestyle issues or poor adherence to dietary guidelines. It is less well known, however, that each of these illnesses, along with many others, might emerge as a result of exposure to adverse childhood experiences in the family environment (Boyce, Sokolowski, \& Robinson, 2012; Hertzman, 2012; Shonkoff, Boyce, \& McEwen, 2009). Applying the developmental ecological perspective presents a useful research strategy as "understanding the multiple etiologies and sequelae of domestic violence requires the use of comprehensive conceptual models that bring together multiple theoretical perspectives under a broad umbrella” (Mohr \& Fantuzzo, 2000, p. 70).

\section{Literature Review Methodology}

In this narrative review of the recent literature, we summarize the current research on the effects on children and youth of the "adversity package" (Jirapramukpitak, Harpham, \& Prince, 2011); that is, the "family burden" (Kassis, Artz, \& Moldenhauer, 2013) of exposure to domestic violence. Domestic violence, or intimate partner violence, can be defined as "patterns of assaultive and coercive behaviors that adults use against their intimate partners" (Holden, 2003, p. 155). We use these terms interchangeably throughout this paper, and use the term "family violence" to reflect both IPV and child abuse and/or maltreatment. Our review examines the specific effects of children's exposure to IPV in a number of important arenas, and the factors that may influence these outcomes. The review is divided into sections that reflect the main areas of research on family violence and child and youth outcomes: (a) neurological disorders; (b) physical health outcomes; (c) mental health challenges; (d) conduct and behavioural problems; (e) delinquency, crime, and victimization; and (f) academic and employment outcomes. We did not include the already well-reviewed and analyzed public health research on intimate partner violence here because this literature focuses rather more on the impacts of IPV on women than on children. In that regard, we refer readers to the following for a comprehensive overview of the 
International Journal of Child, Youth and Family Studies (2014) 5(4): 493-587

In Harm's Way: A Special Issue on the Impacts and Costs of Witnessing Intimate Partner Violence

public health research on the impacts of IPV: Afifi (2011), Black et al. (2008), Public Health Agency of Canada (2010), and Varcoe et al. (2011).

Literature searches for each section of the review were conducted using keywords most relevant to the particular area of focus; for example, “domestic violence” (and synonyms such as family violence, spousal violence, intimate partner violence, inter-parental violence) and children/youth or exposure/witnessing, ${ }^{2}$ in addition to keywords associated with the outcomes of interest (e.g., neurobiology, physiology, health, illness, hospitalization, mental disorder, substance use, addiction, internalizing, externalizing, conduct, behaviour, crime, delinquency, victimization, education, employment). Specific databases searched included: PsycINFO (for neurological, mental health, substance misuse, conduct and behavioural, and delinquency and crime outcomes), PsycArticles (for mental health and substance misuse outcomes), Criminal Justice Abstracts (for mental health, substance misuse, and conduct and behavioural outcomes), Social Work Abstracts (for delinquency and crime outcomes), EBSCO (for employment outcomes), and Summon (for physical health and education outcomes). Google Scholar was also searched for all but two outcome areas (employment and conduct and behavioural problems). The review included English-language articles only and, while the majority were original articles, review articles were also included. The review focused on literature published between 2006 and 2014 in order to capture the latest research on children's exposure to IPV and avoid replicating recent review articles on the subject, which typically report on original research published before the year 2006. Abstracts identified in the literature search were reviewed for relevance and, in most cases, additional articles were identified by scanning the reference lists of articles selected for inclusion. Google Scholar was used to identify any additional articles that may have been missed in the academic database searches.

\section{Neurological Disorders}

A useful departure in accounting for the "biological embedding" of individual life experiences is to view development as a series of interactions between genomic and nongenomic factors in a cascading context of events that require the developing individual to continually respond to changing demands in an adaptive manner (Hertzman, 2012). Although development in humans is exquisitely complex, more so than in even the most advanced nonhuman species, some elementary physiological mechanisms underlying individual variations to early social experiences have probably been conserved (Denver \& Crespi, 2006; Hertzman, 2012; Kolb, Gibb, \& Robinson, 2003; Perry \& Pollard, 1998; Rutter, 2012). Specifically, the most critical neurobiological regulatory networks are the hypothalamic-pituitary-adrenal axis and the autonomic nervous system; together they generate a complex array of adaptive networks of physiological and behavioural responses under stressful conditions that mature over time through close interactions with the physical and social environment. ${ }^{3}$ Therefore, early traumatic

\footnotetext{
${ }^{2}$ The terms “exposure” and "witnessing” are used interchangeably throughout this report when referring to children's experiences in relation to IPV.

${ }^{3}$ The autonomic nervous system consists of two complementary parts, the sympathetic and the parasympathetic nervous systems, functioning through the release of two different hormones, epinephrine and norepinephrine. While the role of the sympathetic nervous system is to prepare the body for rapid metabolic change and physical movement
} 
International Journal of Child, Youth and Family Studies (2014) 5(4): 493-587

In Harm's Way: A Special Issue on the Impacts and Costs of Witnessing Intimate Partner Violence

relationship features not only affect the formation of neural circuits in the brain but get mapped into the structure and function of the neuroendocrine system involved in mediating the timing of life history transitions, as well as in generating immune reactions and integrated behavioural responses to stress (Denver \& Crespi, 2006; Porter et al., 2003). Essentially, "the neuroendocrine system transduces environmental information into developmental and physiologic responses” (Denver \& Crespi, 2006, p. e184).

The pace and quality of this neurological development is governed by the complex interaction between environmental forces and neuroendocrine factors in children. Organ maturation in intrauterine life, including the fetal brain and nervous system, can be sped up by the presence of chronic maternal stressors (e.g., socioeconomic, emotional, or nutritional) stimulating precocious maturation of the fetal neurobiological system that regulates physiological and behavioural responses under stress. The proximate benefit of such processes could presumably mean neurobiological adjustment to conditions demanding constant vigilance and readiness, but the distal cost is significant as the underlying physiological stress-response system is tuned to an environment in which the cost of survival is altered neuroendocrine activities, reduced body size, and lifelong susceptibilities to diseases (e.g., obesity, hypertension, type 2 diabetes), along with negative behavioural and mental conditions (e.g., externalizing and internalizing problems such as aggression, anxiety, and depression) and higher mortality rates (for a summary see Denver \& Crespi, 2006; Perry \& Pollard, 1998).

This research highlights the critical role that early attachment relationships play in the development of neurobiological regulatory mechanisms because elevated cortisol levels early in life are associated with structural and functional impairments in various domains (Danese \& McEwen, 2012; Hertzman, 2012; McCrory et al., 2011). More specifically, Danese and McEwen (2012) outline three brain structures critically affected by elevated cortisol production associated with chronic exposure to severe stress coming from adverse family environments (i.e., allostatic overload). First, anatomical alterations in the prefrontal cortex precipitate a host of behavioural conditions such as impaired attention, and problems with emotional control and executive functioning. Second, structural changes in the amygdala increase the risk of heightened sensitivity to unlearned fear and fear conditioning. Finally, stress-related reduction in the hippocampus is associated with impaired memory processes in the declarative, contextual, and spatial domains. Taken together, findings from animal and human studies provide converging evidence that the stress-response system is a "phylogenetically ancient signaling system" keeping track of salient interpersonal relationships and converting them to specific patterns of underlying neurochemical dynamics (Denver \& Crespi, 2006, p. e187). Given that early adverse experiences from the social environment might have long-term consequences by mapping into the architecture of the developing neurobiological apparatus, the biological cost paid by those

under stressful conditions, the parasympathetic nervous system is associated with opposite tendencies of energy conservation and relaxation. Balance between those two parts of the autonomic nervous system is essential for adaptive functioning. Through the release of a series of different hormones, on the other hand, the hypothalamicpituitary-adrenal axis has a slower but more enduring effect on the body. In general, hypothalamic-pituitary-adrenal activity results in increased metabolic rate in structures required for action (e.g., heart and skeletal muscles) along with suppressed expenditure in those ones that are not of immediate concern under conditions of acute stress (e.g., digestion and immune responses). 
International Journal of Child, Youth and Family Studies (2014) 5(4): 493-587

In Harm's Way: A Special Issue on the Impacts and Costs of Witnessing Intimate Partner Violence

who suffer from these conditions can have far reaching consequences long after the timing of the traumatizing childhood relationship.

\section{Neurodevelopmental Consequences of Childhood Maltreatment}

Based on research on the neurodevelopmental consequences of childhood maltreatment, we know that:

1. Early socialization plays a critical role in the structural and functional development of the brain (Danese \& McEwen, 2012; Grassi-Oliveira et al., 2008; McCrory et al., 2011; Shonkoff et al., 2009; Teicher, 2000).

2. The most influential of all social interactions is the relationship with primary caregivers in the family (Harlow, Dodsworth, \& Harlow, 1965) with direct implications for the emergence of neurobiological architecture in the infant (Anda et al., 2006; Carpenter \& Stacks, 2009; Saltzman et al., 2005; Yount et al., 2011).

3. Being subjected on a regular basis to abusive, neglectful, or severely stressful conditions within the parent-child relationship significantly elevates the risk of experiencing lifelong deterioration both in physical and mental health (Adams, 2006; Cummings, El-Sheikh, Kouros, \& Buckhalt, 2009; Katz, 2007; Rigterink, Katz, \& Hessler, 2010; Roustit et al., 2009).

4. Delayed effects of early adverse experiences on health and well-being are mediated through various neuroanatomical (Choi, Jeong, Polcari, Rohan, \& Teicher, 2012), neuroendocrine (Engert et al., 2010; Sturge-Apple, Davies, Cicchetti, \& Manning, 2012), and epigenetic factors (Essex et al., 2013; McDermott, Dawes, Prom-Wormley, Eaves, \& Hatemi, 2013).

At least four main arguments can be made in favour of widening the breadth of research in the domain of family violence. First, many more children witness the effects of IPV than become the target of domestic violence (Adams, 2006). Second, different forms of abuse might be associated with distinct mechanisms inflicting damage (Margolin \& Vickerman, 2011). Third, although a growing body of literature illustrates the psychosocial and neurodevelopmental sequelae of child abuse and neglect (Anda et al., 2006; Shonkoff, 2003; Wood \& Sommers, 2011), scholars have just recently made attempts to examine how exposure to IPV alters developmental trajectories of the infant (Carpenter \& Stacks, 2009; Yount et al., 2011). Fourth, as deleterious outcomes can be mediated at multiple levels of functioning, experts across diverse disciplines (e.g., social epidemiology, developmental sciences, psychopathology, neurosciences, and molecular genetics) should integrate their efforts to address the issue of domestic violence influencing children's health and behaviour (Margolin \& Vickerman, 2011; Mohr \& Fantuzzo, 2000; Mohr, Lutz, Fantuzzo, \& Perry, 2000).

A minority of research in the area has emphasized that traumatic experiences in the family do not purely evoke pathological patterns of behavioural and emotional issues but also inflict "bruises and scars" (Saltzman et al., 2005, p. 136) in underlying physiological and neuroendocrine mechanisms causing problems which often remain unobservable for years (Adams, 2006). Despite substantial evidence suggesting that structural and functional alteration in the neuroendocrine system probably represents the most fundamental consequence of family violence (Anda et al., 2006; Perry \& Pollard, 1998; Shonkoff, 2003), research in this respect lags 
International Journal of Child, Youth and Family Studies (2014) 5(4): 493-587

In Harm's Way: A Special Issue on the Impacts and Costs of Witnessing Intimate Partner Violence

behind investigations concerned with more observable somatic and psychosocial difficulties associated with children's exposure to IPV (Carpenter \& Stacks, 2009; Yount et al., 2011). The aim of this foundational section of the current review is, therefore, to fill this gap of knowledge by focusing specifically on the neurobiological correlates of early-onset exposure to domestic violence. Several themes arise from a critical review of this body of research including: (a) cortisol production as a measure of variation in hypothalamic-pituitary-adrenal axis activity; (b) patterns of activation in the sympathetic nervous system and parasympathetic nervous system; and (c) other neurobiological correlates (e.g., white matter abnormalities and epigenetic regulation of neurochemical synthesis).

\section{Neurobiological Regulation in Infants Exposed to IPV}

Animal and human studies have provided ample evidence that adverse early environments (e.g., lack of parental care, emotional neglect, insufficient nursing) can cause profound alterations in the activities of the sympathetic nervous system, parasympathetic nervous system, and hypothalamic-pituitary-adrenal axis constituting the fundamental elements of a highly integrated physiological regulatory system evolved to support the individual to effectively deal with imminent demands (Danese \& McEwen, 2012; Kemeny, 2003).

Hypothalamic-pituitary-adrenal axis activation. Eleven of the 26 articles reviewed in this section assessed hypothalamic-pituitary-adrenal axis functions in the context of exposure to IPV focusing on patterns of cortisol production (Davies, Sturge-Apple, Cicchetti, Manning, \& Vonhold, 2012; Davies, Sturge-Apple, \& Cicchetti, 2011; Davies, Sturge-Apple, Cicchetti, \& Cummings, 2007, 2008; Davies, Sturge-Apple, Cicchetti, Manning, \& Zale, 2009; Hibel, Granger, Blair, Cox, \& The Family Life Project Key Investigators, 2009, 2011; Koss et al., 2011; Stride, Geffner, \& Lincoln, 2008; Sturge-Apple et al., 2012; Towe-Goodman, Stifter, MillsKoonce, Granger, \& The Family Life Project Key Investigators, 2012). Cortisol secretion normally increases as a response to physical and psychological stressors, facilitates the mobilization of resources inside the body, and alters the processing of emotionally charged stimuli. Short-term activation of the stress-response system is adaptive with rapid elevation in the cortisol level followed by a return to the normal baseline level. Chronic exposure to stressprovoking conditions such as IPV, on the other hand, evokes an environment where the hypothalamic-pituitary-adrenal system calibrates to address recurrent hostilities associated with long-term dysfunction in cortisol regulation (down-regulation or up-regulation) mediating risk for maladaptive patterns of behavioural adjustment (e.g., externalizing and internalizing problems).

Autonomic nervous system activation. While parental care and the family's emotional atmosphere profoundly affect the structural and functional properties of the hypothalamicpituitary-adrenal axis in the developing infant, this is but one of the three different components of an integrated human stress-response system which is influenced by the quality of the early social environment. El-Sheik and colleagues have recently advanced a model highlighting the relationship between exposure to marital conflicts and subsequent developmental psychopathology modulated by individual variations in the functioning of the child's autonomic nervous system (Cummings et al., 2009; El-Sheikh \& Erath, 2011; El-Sheikh et al., 2009). The most critical element of the theory lies in the proposition that adaptive/maladaptive patterns of 
International Journal of Child, Youth and Family Studies (2014) 5(4): 493-587

In Harm's Way: A Special Issue on the Impacts and Costs of Witnessing Intimate Partner Violence

physiological and psychological activities within a particular social environment are associated with a highly coordinated and hierarchical neurobiological system involving the sympathetic nervous system and parasympathetic nervous system.

As the term neural plasticity implicates (Kolb et al., 2003), regulatory features of the autonomic nervous system emerge during early childhood "in a perpetuating process of coordinated fine-tuning to meet individual and environmental needs” (El-Sheikh et al., 2009, p. 2), shaping individual patterns of emotional and psychosocial characteristics. Consistent with this theoretical position, it is suggested that physical, emotional, or sexual maltreatment in the family may result in early physiological and emotional "fine-tuning" to a hostile life-course environment giving rise to behavioural strategies (externalizing and internalizing) not best suited under less hostile social conditions (Cummings et al., 2009). Nonetheless, individual variations in emotional and physiological responsiveness may also moderate the effect of early maltreatment on children’s behaviour (Ellis \& Boyce, 2011; Obradović \& Boyce, 2009). Gordis, Feres, Olezeski, Rabkin, and Trickett (2010) have demonstrated, for example, that increased parasympathetic activity measured at the baseline in 12-year-old boys in a laboratory challenge task serves as a protective factor moderating the deleterious effect of an abusive family environment on aggressive tendencies. Conversely, other studies indicate that children displaying evidence of diminished fearfulness and behavioural disinhibition more likely manifest externalizing symptoms in the context of harsh parenting if they show signs of lowered sympathetic reactivity (Cummings et al., 2009).

Thirteen of the 26 studies reviewed in this section examined whether witnessing domestic violence was associated with alterations in the development of autonomic regulatory functions. Physiological indices of sympathetic nervous system were identified as measures of skin conductance level (El-Sheikh, Hinnant, \& Erath, 2011; El-Sheikh, Keiley, Erath, \& Dyer, 2013; El-Sheikh, Keller, \& Erath, 2007; El-Sheikh et al., 2009), salivary alpha amylase (Gordis, Margolin, Spies, Susman, \& Granger, 2010), and cardiac activity (Davies et al., 2009; Stride et al., 2008). The same indices for parasympathetic nervous system function included measures of vagal activity (Davies et al., 2009; El-Sheikh \& Hinnant, 2011; El-Sheikh et al., 2011; El-Sheikh et al., 2013; El-Sheikh et al., 2009; El-Sheikh \& Whitson, 2006; Katz, 2007; Moore, 2010; Obradović, Bush, \& Boyce, 2011; Rigterink et al., 2010). Six of the 13 papers reported registering more than one physiological marker of neurobiological regulation (Davies et al., 2009; El-Sheikh et al., 2011; El-Sheikh et al., 2013; El-Sheikh et al., 2009; Obradović et al., 2011; Stride et al., 2008).

Three cross-sectional studies directly tested sympathetic responses in the context of childhood exposure to marital violence (Davies et al., 2009; Gordis, Margolin, et al., 2010; Stride et al., 2008). Two of them (Gordis, Margolin, et al., 2010; Stride et al., 2008) provide support for the sensitization hypothesis predicting increased sympathetic nervous system activation associated with repeated exposure to marital conflict (El-Sheikh et al., 2009). Using college students' retrospective reports on childhood exposure to abusive environments, for example, Stride, Geffner, and Lincoln (2008) found significantly higher levels of heart rate to stressprovoking conditions in students reporting IPV exposure compared to those reporting no abusive experiences. This result is of particular interest as no other indices used in the study (e.g., cortisol 
International Journal of Child, Youth and Family Studies (2014) 5(4): 493-587

In Harm's Way: A Special Issue on the Impacts and Costs of Witnessing Intimate Partner Violence

and behavioural trauma symptoms) yielded comparable difference with exposure to IPV versus no abuse. Contrary to the sensitization hypothesis, on the other hand, Davies and colleagues (2009) obtained diminished basal sympathetic nervous system activity in 27-month-old toddlers exposed to IPV.

Taken together, several pieces of the data reviewed above lend limited support for the view that early experiences of IPV could be associated with emerging regulatory patterns aiming to enhance sensitivity to physiological and behavioural readiness. Specifically, witnessing marital discord was associated with: (a) enhanced sympathetic activity at rest (Stride et al., 2008) and under conditions of stress (Gordis, Margolin, et al., 2010); (b) diminished parasympathetic activity at baseline and during a task requiring active engagement along with attenuation of withdrawal of parasympathetic activity in situations involving emotional stress (Moore, 2010); and (c) reduced increase of parasympathetic tone at baseline during middle childhood years ${ }^{4}$ (Rigterink et al., 2010). This line of evidence is consistent with some neurobiological implications of emotional security theory (see Cummings et al., 2009) insofar as this theory considers children's evolving pattern of self-regulatory capacity as an important conduit where experiencing emotional security in the family has an impact on subsequent adjustment functions in life. More specifically, a central tenet of emotional security theory is the proposition that repeated experiences of stressful family conditions, such as marital violence, give rise to emotional insecurity and thereby generate conditions for allostatic ${ }^{5}$ load (the wear and tear on the body which grows over time when the individual is exposed to repeated or chronic stress), thus reflecting "the price the body pays for being forced to adapt to adverse psychological and physical situations [...] or the inefficient operation of the allostasis response systems” (McEwen, 2000, p. 174). Experiencing IPV can “tune” an infant’s neurobiological apparatus to a developmental trajectory characterized by enhanced sympathetic reactivity and concurrently reduced parasympathetic reactivity, generating a state of perpetual physiological readiness (or "sensitization", see El-Sheikh \& Whitson, 2006) even when no challenge is actually present. At the same time, this developmental trajectory leads to experiencing insufficient mobilization of physiological resources when coping is required (i.e., dampened elevation of sympathetic nervous system accompanied by impaired suppression, or elevation, of parasympathetic nervous system activation to stress). This means the individual is both overreactive and unable to respond (i.e., frozen in a state of readiness to fight).

A significant proportion of the data reviewed above, however, remains inconsistent with the sensitization model of autonomic regulations (Davies et al., 2009; El-Sheikh \& Hinnant, 2011; El-Sheikh et al., 2011; El-Sheikh et al., 2013; El-Sheikh et al., 2009; El-Sheikh \& Whitson, 2006). It is conceivable, on the other hand, that attenuation - instead of augmentation - of baseline readiness develops as part of physiological regulation in the context of marital conflict. As Davies et al. (2009) have suggested, "lowering of the set point of the [sympathetic nervous system] may specifically reflect the activation of processes designed to prevent the toxic effects

\footnotetext{
${ }^{4}$ The normative developmental pattern of autonomic regulatory functions includes gradual elevation of resting parasympathetic nervous system tone over the years of childhood.

${ }^{5}$ Allostasis refers to adaptive variations in physiological outputs (e.g., heart rate and blood pressure) emphasizing the body's natural ability to regulate different "allostatic” response systems in the service of meeting ambient environmental demands.
} 
International Journal of Child, Youth and Family Studies (2014) 5(4): 493-587

In Harm's Way: A Special Issue on the Impacts and Costs of Witnessing Intimate Partner Violence

of the chronic overarousal in response to threat” (p. 1389). Heightened and lowered sensitivities of autonomic self-regulation, however, incur different health costs: Heightened and lowered levels of arousal are linked to internalizing and externalizing symptoms, respectively (Davies et al., 2009).

Individual differences in neurobiological sensitivity. Research discussed so far provides strong evidence that children's experiences of parental discord in the early rearing environment are associated with altered neurobiological development resulting in increased odds for negative life outcomes. Nonetheless, these findings are insufficient to account for the observation that not every child is equally affected by early adverse influences in the family (Belsky, BakermansKranenburg, \& van IJzendoorn, 2007; Gordis, Feres, et al., 2010). One common explanation regarding these discrepancies implies that a particular risk factor (e.g., maltreatment in the family) can be considered as a necessary, but not sufficient, cause of child maladjustment. In other words, a single risk factor usually co-acts interactively with multiple contributors (e.g., genetic, neurobiological, psychosocial, cultural) to produce a developmental outcome in any individual (Cicchetti \& Rogosch, 1996). For instance, although two infants might be equally exposed to early maltreatment, the one that exhibits symptoms of adjustment issues might carry a particular form of genetic polymorphism associated with increased vulnerability to maladaptive behaviours under specific adverse environmental influences (Bakermans-Kranenburg \& van IJzendoorn, 2011; Belsky et al., 2007).

Mona El-Sheikh's recent research program represents one line of study that has applied a multi-system approach to examine patterns of sympathetic nervous system and parasympathetic nervous system activities together to explain the pathways of individual variations in sensitivities to maladaptive consequences of early exposure to IPV (Cummings et al., 2009; El-Sheikh \& Erath, 2011; El-Sheikh et al., 2009). In short, the underlying idea is that physiological systems, like the sympathetic nervous system and parasympathetic nervous system, operate in concert. In other words, elements of physiological regulation must function in a synchronized fashion to ensure adaptive behaviour and emotional stability. As autonomic regulation is not yet fully shaped in infancy, chronic stress experienced in the family could result in dysregulated and uncoordinated patterns of sympathetic and parasympathetic nervous system activities that have the potential to forecast problematic behavioural and mental symptoms - that is, externalization and internalization issues - later in life. Most importantly, particular patterns of physiological regulation can moderate deleterious effects of IPV on child adjustment (Davies et al., 2007).

Findings of three independent cross-sectional studies by El-Sheikh and colleagues reported in the Monograph of the Society for Research in Child Development summarize four different profiles of physiological regulation explaining individual differences in vulnerability of behavioural symptoms within the framework of domestic stress (El-Sheikh et al., 2009). More specifically, low sympathetic activation in children (aged 6 to 12 years) was a vulnerability factor for problem behaviours measured in the context of decreased parasympathetic activation (co-inhibition). In contrast, low sympathetic activation was a protective factor in infants whose concurrent parasympathetic activation was elevated (reciprocal parasympathetic activation). Similarly, co-activation (high sympathetic nervous system reactivity and high resting parasympathetic nervous system) and reciprocal sympathetic activation (high sympathetic 
International Journal of Child, Youth and Family Studies (2014) 5(4): 493-587 In Harm's Way: A Special Issue on the Impacts and Costs of Witnessing Intimate Partner Violence

nervous system reactivity and low resting parasympathetic nervous system tone) would render infants more and less vulnerable, respectively, to behavioural problems in the context of marital conflicts.

A recent longitudinal study conducted by El-Sheikh's group has found evidence of gender difference in children (aged 8 to 10 years) in the relationship between autonomic regulatory patterns and behavioural adjustment issues within the framework of domestic stress (El-Sheikh et al., 2013). For example, co-inhibition in girls was a vulnerability factor for behavioural symptoms at each time point of the study if they were exposed to high family conflict. In contrast, co-inhibition in high family conflict environments predicted the sharpest decline of adjustment problems in boys by the age of 10. It is important to note, however, that the two studies published by El-Sheik et al., (2013; 2009) used different measures of problem behaviour as the former was focused on externalizing problems (e.g., non-compliance and aggression) whereas the latter assessed internalizing problems (e.g., depression and anxiety).

The work conducted by El-Sheikh et al., (2013; 2009) is consistent with ecological (Mohr \& Fantuzzo, 2000; Mohr et al., 2000) and contextual (Steinberg \& Avenevoli, 2000) perspectives of developmental psychopathology documenting that adjustment problems in children who are exposed to IPV should not be considered as a simple function of toxic environmental conditions. Instead, as noted by Cicchetti and Rogosch (1996), "the meaning of any one attribute, process, or psychopathological condition needs to be considered in light of the complex matrix of individual characteristics, experiences, and social-contextual influences involved, the timing of events and experiences, and the developmental history of the individual” (p. 599). Thus, for instance, increased parasympathetic nervous system activation to a laboratory challenge, in conjunction with low sympathetic nervous system responsiveness, can actually serve either as risk for, or protection from, symptoms of behavioural maladjustment in girls from high-conflict homes and boys from low-conflict homes, respectively (El-Sheikh et al., 2013). A plausible interpretation for such a finding is that interaction among elements of the neuroendocrine regulatory system reflecting organismic variables as well as the emotional quality of the early rearing environment engenders a "psychobiological fingerprint" (Boyce et al., 2001, p. 148) for individual variations in context-specific stress-response matrices.

The second avenue of research directly addresses the role of contextual modulation in the relationship between parasympathetic response patterns and developmental consequences of child maltreatment. As a whole, higher resting parasympathetic nervous system activation (i.e., keeping sympathetic control under check) and greater parasympathetic nervous system suppression to challenge (i.e., easing parasympathetic "break" over sympathetic activation) should buffer against negative developmental outcomes (Cummings et al., 2009; El-Sheikh \& Erath, 2011), ensuring adaptive neurobiological regulation and readiness to "greater social engagement, exploration, activity, expression of empathy and positive emotion, fewer behavioral problems, better emotional regulation, and less antisocial behavior" (Cipriano, Skowron, \& Gatzke-Kopp, 2011, p. 206). However, several authors report, on the other hand, that elevated parasympathetic tone at baseline is not a protective factor but actually a risk for problem behaviour if the child is exposed to community violence (Scarpa, Tanaka, \& Chiara Haden, 2008). To underscore the importance of parasympathetic activity in behavioural adjustment, it 
International Journal of Child, Youth and Family Studies (2014) 5(4): 493-587

In Harm's Way: A Special Issue on the Impacts and Costs of Witnessing Intimate Partner Violence

has been suggested that parasympathetic nervous system control of emotional regulation in childhood reflects a general sensitivity to variations in environmental conditions rather than a stable trait-like emotional predisposition (Cipriano et al., 2011).

To summarize, childhood exposure to IPV does not unconditionally lead to deleterious developmental outcomes but individual and contextual variations are thoroughly implicated in the process. Most importantly, a multisystem approach using concurrent measures of physiological parameters (e.g., parasympathetic nervous system and sympathetic nervous system) seems to be the most promising as biological regulation is based on integrated activation of different regulatory systems. Additionally, physiological systems do not work in a vacuum but exert their influence in particular contexts. Hence interactions between contextual parameters (e.g., high-stress and low-stress family environments) and patterns of integrated neurobiological activities are likely to determine individual trajectories of responsiveness in the case of witnessing marital violence at home.

Structural and epigenetic alterations in the brain. The evidence reviewed so far illustrates that not only can abuse targeting the child be associated with severe developmental outcomes, but also that sole exposure to parental violence (i.e., witnessing family aggression) on a regular basis may be related to impairments in emotional, psychosocial, health-related, and neurobiological functioning. With the advent of improved technology introduced in the neurosciences, novel approaches to the study of neural development can increase our understanding of brain regions that are most affected by the harmful effects of child maltreatment (Grassi-Oliveira et al., 2008; McCrory et al., 2011; Nelson, 2007; Teicher, 2000). For example, employing positron emission tomography in studying brain development among Romanian orphans suffering from severe early neglect and social deprivation has produced one of the first brain imaging results of chronic childhood maltreatment. These results showed decreased metabolism in the prefrontal and temporal areas and other regions in the brain associated with higher cognitive functions and emotional control, thus corroborating behavioural data showing neurocognitive impairments in these orphans along with reduced attention and socio-emotional problems (Nelson, 2007). Such findings provide strong evidence that severe early abuse and neglect appear to lead to anatomic and functional alterations in the young brain with profound implications to developmental pathways associated with adjustment issues in various domains of functioning. There are only a few studies, however, inquiring about comparable effects on brain development by simply witnessing IPV on a regular basis at home during the infant years.

Three recent papers published by Martin Teicher and his team focus specifically on the relationship between witnessing parental discord by children and neuropsychiatric consequences (Choi et al., 2012; Teicher, Samson, Sheu, Polcari, \& McGreenery, 2010; Teicher \& Vitaliano, 2011). Each study used a proxy measure termed "limbic irritability" (Teicher, Tomoda, \& Andersen, 2006). The underlying idea is that development of the limbic system - a set of interconnected subcortical structures involved in a variety of regulatory functions - would be particularly sensitive to exposure to heightened levels of stress. Stress-induced chronic stimulation of the limbic system due to exposure to domestic violence should, therefore, exert a negative influence on the development of the limbic area in the brain (Teicher et al., 2010; 
International Journal of Child, Youth and Family Studies (2014) 5(4): 493-587

In Harm's Way: A Special Issue on the Impacts and Costs of Witnessing Intimate Partner Violence

Teicher \& Vitaliano, 2011). Abnormal development in the limbic system, in turn, is considered a risk factor for adult neuropsychiatric malfunctions including brief hallucinatory events, distortions in the somatic and perceptual domains, dissociative symptoms and motor automatisms (e.g., involuntary, stereotyped, and non-purposeful movement of the upper extremities). Data presented in the three articles indicate that witnessing IPV during childhood can influence limbic irritability in a complex manner. Most germane to this review are the findings that retrospective reports of young adult participants on witnessing IPV, coupled with parental verbal abuse, appeared to generate higher levels of limbic irritability scores than sole exposure to physical or sexual abuse (Teicher, Samson, Polcari, \& McGreenery, 2006). In contrast, Teicher and Vitaliano (2011) found no direct association between witnessing violence toward the mother or the father and limbic irritability. Nonetheless, this does not mean that no relationship exists between these factors. Conversely, by conducting a series of path analyses to detect interrelationships between different forms of maltreatment and psychiatric outcomes, Teicher and Vitaliano (2011) have found evidence that the association between witnessing family violence targeting the mother and limbic irritability is mediated by maternal verbal aggression. The authors explain this complex phenomenon suggesting "that domestic violence toward [the] mother affects the emotional well-being of her children by primarily altering her behavior, which may be reflected in her more frequent use of verbal aggression” (p. 9).

In the third study, Choi, Jeong, Polcari, Rohan, and Teicher (2012) employ sophisticated image acquisition tools and analyzing techniques to detect structural alterations in the brain following early exposure to IPV. Retrospective reports of young adults on witnessing domestic violence during childhood, combined with magnetic resonance imaging examinations of the brain and neuropsychological assessments, revealed a link between domestic violence and the anatomic development of a bundle of fibres in the brain (i.e., inferior longitudinal fasciculus) central in emotional regulation and processing sensory information on adverse events between different cortical regions. The authors found that symptoms of limbic irritability were associated with both IPV and inferior longitudinal fasciculus anatomy. Most importantly, however, exposure to verbal disputes between parents was a stronger predictor of attenuation in the size of inferior longitudinal fasciculus than inter-parental physical violence.

Taken together, the three studies conducted by Teicher and colleagues converge upon four major points:

1. Early exposure to stressful social conditions in the form of domestic violence is capable of sculpting postnatal brain development.

2. Reduction in the volume of both the white (Choi et al., 2012) and grey matter (Teicher et al., 2010) may alter the way the brain processes sensory information to increase the person's vulnerability to psychopathology.

3. Although the harmful effects of child maltreatment are typically defined in terms of physical or sexual harassment and emotional neglect, verbal abuse appears to be a powerful modifying factor for neural development in the young brain regardless of whether the child is the target of peer (Teicher et al., 2010) or parental (Choi et al., 2012) verbal abuse, or witnesses domestic verbal conflicts (Choi et al., 2012). 
International Journal of Child, Youth and Family Studies (2014) 5(4): 493-587

In Harm's Way: A Special Issue on the Impacts and Costs of Witnessing Intimate Partner Violence

4. The statistically insignificant association between IPV and psychiatric outcomes should not lead us to believe that experiencing domestic violence has no impact on development because this relationship might be potentiated (mediated) by effects from other powerful determinants (Teicher \& Vitaliano, 2011).

\section{Physical Health Outcomes}

The health and safety of children is intricately linked to the health and safety of their mothers, particularly in families where violence is present. IPV has been widely recognized as a public health epidemic, given the strong evidence that violence against women has a negative impact on women's physical and mental health and well-being (Chrisler \& Ferguson, 2006; Garcia-Moreno \& Watts, 2011). Exposure to violence and trauma is associated with a number of severe negative health outcomes, and domestic violence in particular has been linked to negative health and medical outcomes for women. For example, research suggests that the health status of women who have experienced IPV is lower than that of other women, and that the health care system incurs significant costs associated with treating violence-related health problems (BC Society of Transition Houses, 2011). Yet, women who experience violence often face numerous barriers to health care services, particularly marginalized or minority women experiencing intersecting health and social inequities (Rodríguez, Valentine, Son, \& Muhammad, 2009). And while much is known about post-traumatic stress responses, depression, and other mental health impacts of IPV, less is known about the physical health impacts of violence against women (Olofsson et al., 2011); even less still is known about physical health outcomes for children and youth who are exposed to IPV.

A growing body of literature is beginning to shed light on the incidence and impacts of children's exposure to domestic violence. For example, Bair-Merritt et al. (2008), in their study of domestic violence screening in an urban paediatric outpatient clinic found that, of the 30 women who disclosed domestic violence, over half reported that at least one of their children had watched and/or heard this violence. Specifically, 40\% of the children had heard violence in the home, 33\% had witnessed physical/verbal abuse directly, 27\% had learned about violence in the home from family or friends, $13 \%$ had seen the impact of domestic violence (e.g., injuries or damage to property), and $10 \%$ had experienced life changes as a result of domestic violence. The effects of chronic stress on children who are exposed to domestic violence may increase their vulnerability to health and medical problems and illness (Howell \& Graham-Bermann, 2011), and indeed, research has found that adolescents who have been exposed to domestic violence report poorer overall health (Lepistö, Luukkaala, \& Paavilainen, 2010).

Exposure to IPV is associated with a wide range of negative health outcomes for children and youth, leading to actual or "perceived physical illness" (Fredland, Campbell, \& Han, 2008, p. 164), chronic disease, and in some cases premature death (Brown et al., 2009). The direct effects of violence exposure include psychological distress associated with witnessing violence against a child's primary caregiver, often the child's mother, or witnessing the aftermath of this violence (Ackerson \& Subramanian, 2009; Anda et al., 2006; Bair-Merritt et al., 2008). 
International Journal of Child, Youth and Family Studies (2014) 5(4): 493-587 In Harm's Way: A Special Issue on the Impacts and Costs of Witnessing Intimate Partner Violence

Research has shown that infants and children who are exposed to IPV have disrupted adrenocortical activity, and can experience either heightened or lowered cortisol output (BairMerritt, Johnson, Okelo, \& Page, 2012; Saxbe, Margolin, Spies Shapiro, \& Baucom, 2012; Towe-Goodman et al., 2012). For example, the adrenocortical and behavioural stress-response patterns of 7-month-old infants exposed to severe interparental violence exposure is associated with significantly greater odds of high cortisol reactivity and moderate negative behavioural problems compared to infants exposed to no, or low, interparental violence $(p<.05)$ (ToweGoodman et al., 2012). In turn, chronic stress associated with living in a home where domestic violence is occurring may increase children's vulnerability to other health and medical problems (Herman-Smith, 2013; Howell \& Graham-Bermann, 2011).

Children who are exposed to IPV also have significantly more eating, sleeping, and pain complaints than children who have not been exposed to domestic violence (Lamers-Winkelman, Schipper, \& Oosterman, 2012). In their recent Dutch study, Lamers-Winkelman and colleagues (2012) compared the physical complaints reported by caregivers of children are exposed to IPV (ages 6 to 12 years) with the somatic complaints of a matched sample. Their results showed that the exposed children experienced significantly more somatic health complaints than those without exposure. For instance, the parents of IPV-exposed children reported that their children had significantly more eating complaints (i.e., eats too much, overweight, constipation, and nausea), sleeping complaints (i.e., overtired, trouble sleeping, and nightmares), and pain problems (i.e., aches and pains, headache, stomach ache, and dizziness). Further, this study showed that there were few differences in health complaints between children who experienced only IPV exposure, and those with compounded experiences of maltreatment (e.g., IPV and physical abuse or other trauma).

Negative health outcomes for children who are exposed to domestic violence may also impact an individual's health over his or her lifetime. In a 26-year long-term prospective population-based study in Sweden, researchers found that when controlling for confounding variables (e.g., adult violence exposure and health), late adolescent IPV exposure significantly increased the women's odds for self-reported bad health and heavy illness burdens in adulthood when compared to non-IPV-exposed women (Olofsson, Lindqvist, Shaw, \& Danielsson, 2012).

Negative health outcomes associated with domestic violence exposure may also be a result of the impact of IPV on women's mental and physical health, which in turn can affect their ability to provide proper care for their children (Åsling-Monemi, Naved, \& Persson, 2008; Hasselmann \& Reichenheim, 2006; Olofsson et al., 2011; Silverman et al., 2011). Additionally, if mothers are experiencing domestic violence, they may be less likely to seek health care for their children for fear that health care practitioners may detect and report domestic violence to the authorities (Ackerson \& Subramanian, 2009; Onyskiw, 2002; Rico, Fenn, Abramsky, \& Watts, 2011). Onyskiw (2002) noted that children who had witnessed family violence were, in fact, more likely to have had contact with medical and health professionals, but not paediatricians. Other scholars have suggested that women experiencing IPV may use health care services to a greater extent due to "hypervigilance and misinterpretation of symptoms" (BairMerritt et al., 2008, p. 135) or because of difficulties experienced coping with their children's health concerns. 
International Journal of Child, Youth and Family Studies (2014) 5(4): 493-587

In Harm's Way: A Special Issue on the Impacts and Costs of Witnessing Intimate Partner Violence

Poor health outcomes associated with domestic violence are compounded by poverty and poor socioeconomic status, which may further impact women's ability to provide proper care for their children (Ramiro, Madrid, \& Brown, 2010). Exposure to domestic violence may also be an indicator that children are themselves experiencing abuse (Ackerson \& Subramanian, 2009; Olofsson et al., 2011), which is also associated with serious negative health outcomes (Hasselmann \& Reichenheim, 2006).

\section{Health Conditions and Use of Health Services}

Much of the literature on the health impacts of children's exposure to IPV also draws on findings from the Adverse Childhood Experiences (ACE) study on the neurological impacts of exposure to violence in the family described in the previous section. This study involved 15,472 participants over a 3-year period and assessed eight adverse experiences: (a) emotional abuse, (b) physical abuse, (c) sexual abuse, (d) exposure to domestic violence, (e) growing up with household substance abuse, (f) growing up with an adult with mental illness, (g) growing up with a criminal household member, and (h) parental separation or divorce (Anda et al., 2006). Participants received an ACE score that reflected the number of adversities they had experienced during childhood. For example, an ACE score of one indicates that the participant experienced one type of adverse childhood experience, an ACE score of two indicates that the participant experienced two types of adverse childhood experiences, and so on. These ACE scores were used to examine the relationship or association between childhood adversity and a wide range of outcomes measured later in life.

Several reports on findings from the ACE study touch on physical health outcomes associated with domestic violence exposure (Anda et al., 2008; Anda et al., 2006; Brown et al., 2010; Brown et al., 2009; Dube et al., 2009; Ramiro et al., 2010). In many cases, variables of interest were found to have a dose-response, or graded, relationship. An important finding in this growing body of research is that the risk of negative health impacts increases steadily with the number of adverse childhood experiences (Anda et al., 2006). For example, individuals who had one adverse childhood experience were 1.2 times as likely as those with no adverse childhood experiences to have sleep disturbances in adulthood, whereas individuals with four or more adverse childhood experiences were 2.1 times more likely to have sleep disturbance problems than those with no adverse childhood experiences. Similarly, persons with more adverse childhood experiences were more likely to report severe obesity and other physical symptoms. Having more adverse childhood experiences was also associated with increased risk of smoking, illicit drug use, and alcoholism. Other outcomes that reflected a dose-response relationship included headaches (Anda, Tietjen, Schulman, Felitti, \& Croft, 2010), lung cancer (Brown et al., 2010), chronic obstructive pulmonary disease or COPD (Anda et al., 2008), autoimmune diseases (Dube et al., 2009), and as noted above, premature death (Brown et al., 2009). In another study, adults reporting five or more ACEs were 2.6 times more likely to develop COPD, twice as likely to be hospitalized for COPD, and 1.6 times more likely to be taking prescription medication for COPD than those with no reported adverse childhood experiences (Anda et al., 2008). Data from hospital and mortality records revealed that persons with six of more ACEs 
International Journal of Child, Youth and Family Studies (2014) 5(4): 493-587 In Harm's Way: A Special Issue on the Impacts and Costs of Witnessing Intimate Partner Violence

were at 2.7 times the risk of developing lung cancer, and died on average 20 years earlier, than those who had not experienced any adverse childhood experiences (Brown et al., 2009).

Reports based on data from the ACE studies (Anda et al., 2008; Anda et al., 2006; Brown et al., 2010; Brown et al., 2009; Dube et al., 2009; Ramiro et al., 2010) are helpful in illustrating the relationship between childhood adversity and various negative outcomes; however, few of these studies explored the increased risk of outcomes associated with specific adverse childhood experiences. That is, few examined the independent association between exposure to domestic violence and negative health outcomes. Of course, this limitation in the literature reflects the reality that adverse childhood experiences often co-occur and negative outcomes can often not be attributed to a single factor or childhood experience. Still, some studies did report on outcomes associated with domestic violence exposure alone: Lepistö and colleagues (2010) found that, among students aged 14 to 17, witnessing domestic violence during childhood was associated with self-perceived health problems. Olofsson et al. (2011) revealed that Swedish children who had witnessed IPV against their mothers reported poorer overall health, more symptoms, and higher rates of health care system and prescription medication use than children not exposed to IPV. Onyskiw (2002) reported similar findings in Canada: that is, that children who had been exposed to family violence were more likely to have health conditions, poor health status, and to be taking prescription medications than those not exposed to domestic violence.

Several studies have also found a relationship between IPV exposure and asthma in children (Bair-Merritt et al., 2012; Kuhlman, Howell, \& Graham-Bermann, 2012; Subramanian, Ackerson, Subramanyam, \& Wright, 2007; Suglia, Enlow, Kullowatz, \& Wright, 2009). Additionally, a cross-sectional health survey from India using a large nationally representative sample of 88,513 ever-married women revealed an increased risk of asthma in infants of all ages living in the same household where the mother is subjected to IPV (Subramanian et al., 2007). This study found that any individuals exposed to domestic violence against women in the household were at greater risk of developing asthma than those who were not exposed to domestic violence (OR 1.15 to 1.19). This risk was even higher for children under age 5 whose mothers reported experiencing physical IPV in the past year (OR 1.32) and children aged 5 to 14, for whom the risk of asthma was increased by 17\% (Subramanian et al., 2007).

Further research on the impact of adverse childhood experiences on adults revealed that those who had witnessed domestic violence during childhood were 1.3 times more likely to report frequent headaches than those who had not witnessed domestic violence (Anda et al., 2010). In the same vein, Rico et al. (2011) found that adults who had witnessed their mothers being treated violently during childhood were found to be 1.3 times more likely to smoke, 2.4 times more likely to have initiated smoking at an early age, 1.7 times more likely to use alcohol, 1.3 times more likely to drive while under the influence of alcohol, 1.7 times more likely to use illicit substances, more than twice as likely to become pregnant early, 1.5 times more likely to have an unintended pregnancy, and 1.8 times more likely to attempt suicide than individuals who had not been exposed to domestic violence in childhood. It is notable that in Rico et al.'s study, $64 \%$ of participants who had experienced physical abuse as children, and $41 \%$ of those who experienced sexual abuse as children, also reported witnessing domestic violence. This provides 
International Journal of Child, Youth and Family Studies (2014) 5(4): 493-587

In Harm's Way: A Special Issue on the Impacts and Costs of Witnessing Intimate Partner Violence

additional evidence that children who are exposed to domestic violence are often also the victims of child abuse and that both experiences contribute significantly to poor physical health.

Not only has research found an association between exposure to IPV and negative health outcomes, such exposure has also been linked to the greater use of health services. The children of women victims of IPV, especially severe IPV (e.g., being kicked, beaten, choked, or threatened with a weapon), are more likely to use health care services and take prescription medications than children who have not been exposed to domestic violence (Olofsson et al., 2011), and more likely to experience higher rates of emergency room visits at 18 months (RR 2.0) and 36 months (RR 1.9) (Bair-Merritt et al., 2008). However, Olofsson and colleagues (2011) also note that emergency room visits were not greater at follow-up for children whose female caregivers had experienced "minor" (e.g., pushed, slapped, shoved) or no IPV at baseline (RR 0.2).

\section{Nutritional Status and Mortality}

Research on children's exposure to domestic violence and malnutrition has found that the risk of child malnutrition increases as verbal and physical aggression between parents increases such that severe physical aggression between parents is associated with severe and acute malnutrition among children (Hasselmann \& Reichenheim, 2006). Rico et al. (2011), who conducted a five-country study (encompassing Egypt, Honduras, Kenya, Malawi, and Rwanda) on the associations between maternal experiences of intimate partner violence and child nutrition and mortality found a significant association between severe growth stunting due to malnutrition and exposure to IPV in Honduras, Kenya, and Malawi. Research conducted by Sobkoviak, Yount, and Halim (2012) in Liberia produced results similar to those found by Rico et al. and also showed that IPV against women is associated with increased risk for children of stunting $(\mathrm{OR}=2.3)$ and being underweight $(\mathrm{OR}=2.57)$. Both stunting and being underweight are indicators of nutritional status (Sobkoviak et al., 2012). Interestingly, these outcomes may not only be observed in low income and politically stressed countries, such as Honduras, Kenya, and Malawi, as nutritional status can also play a role in young adult life in countries where food is readily available. Witnessing family violence (not only IPV, but other forms as well) has been shown to be moderately but significantly correlated with disordered eating among university students $(r=.24, p<.01)$, with the relationship partially mediated by symptoms of depression and anxiety that may be associated with family violence exposure (Brady, 2008).

At the extreme end of the continuum of negative physical health outcomes associated with exposure to IPV is premature death. A study in India of children under 5 years of age found that physical IPV against women was associated with child mortality $(R R=1.21)$, although this relationship was not found to be as strong for sexual and psychological forms of IPV (Ackerson \& Subramanian, 2009). Findings from this study suggested that regardless of gender, exposure to multiple types of IPV was strongly associated with infant death $(\mathrm{RR}=1.49)$ and somewhat associated with child death $(\mathrm{RR}=1.18)$. An additional study conducted in India confirmed that infant and child mortality is associated with exposure to IPV against women, but in this study the results were confirmed only for girls (Silverman et al., 2011). Silverman et al. explain the girlsonly result by suggesting that girls' exposure to domestic violence is likely compounded by 
International Journal of Child, Youth and Family Studies (2014) 5(4): 493-587

In Harm's Way: A Special Issue on the Impacts and Costs of Witnessing Intimate Partner Violence

gender-based maltreatment and neglect due to gender discrimination in India, and argue that reducing IPV in the country could result in a $6.67 \%$ reduction in female infant deaths and $6.25 \%$ reduction in female child deaths in India.

The five-country study conducted by Rico et al. (2011) described above also examined the mortality of children under 2 years of age and found a significant association between exposure to physical and sexual IPV and mortality in Honduras, Kenya, and Malawi. In contrast, research in rural Bangladesh found no association between IPV against women and mortality for children under age 5, more generally (Åsling-Monemi et al., 2008). However, a closer look at the data gathered by Åsling-Monemi and colleagues (2008) revealed an elevated risk of death for female children of more educated women who were experiencing severe physical violence $(\mathrm{RR}=$ 2.2) and controlling behaviours $(\mathrm{RR}=2.5)$ in their intimate relationships. Åsling-Monemi et al. suggest that these findings may be explained as reflecting differences in socioeconomic status and perceptions of controlling behaviour in marriage between more and less educated women and by gender discrimination and differences in child caring practices for boys and girls in Bangladesh.

The health impacts of exposure to IPV have also been extensively investigated in the area of mental health and substance use. This research is reviewed next.

\section{Mental Health Challenges: Internalizing and Externalizing Issues}

In Canada, child and youth mental health challenges are highly prevalent with $14 \%$ to $25 \%$ of youth presenting with diagnostic symptomatology for at least one mental illness. In fact, two-thirds of adults living with mental concerns indicate that their symptoms began during adolescence (Mental Health Commission of Canada, 2012). The term mental illness covers a broad range of symptoms that interfere with emotions, thinking and/or behaviour, and that contribute to ongoing limitations or impairment (Manion, 2010; Mental Health Commission of Canada, 2012). These vulnerabilities often lead to illness and disability across the lifespan, and beget poor social, economic, and interpersonal outcomes (Manion, 2010; Mental Health Commission of Canada, 2012).

Much of the research on the impact of exposure to IPV focuses on what are referred to as internalizing and externalizing problems. The externalizing construct refers to the outward expression of harmful behaviours (e.g., aggression, hyperactivity, defiance) that affect the external environment. Due to the observable dysregulated emotional states and related conflict that ensues, externalizing symptoms are often referred to as behaviour problems by professionals working with children and youth (Lane, Oakes, Carter, Lambert, \& Jenkins, 2013; Liu, 2004; Mikolajewski, Allan, Hart, Lonigan, \& Taylor, 2013). In the American Psychiatric Association’s (2013) Diagnostic and Statistical Manual of Mental Disorders (DSM-5), the clusters of externalizing symptoms are commonly categorized as oppositional defiant disorder (ODD), conduct disorder (CD), and attention-deficit hyperactivity disorder (ADHD).

While focusing on the internalized and externalized manifestations of mental health and illness, the literature also recognizes the relationship between people and their environment and 
International Journal of Child, Youth and Family Studies (2014) 5(4): 493-587

In Harm's Way: A Special Issue on the Impacts and Costs of Witnessing Intimate Partner Violence

therefore investigates not only the traits of individuals but also the environmental factors that contribute to mental illness (Canadian Association of Social Workers, 2013; Mental Health Commission of Canada, 2012). Thus, the developmental psychopathology perspective posits that mental health challenges arise from complex biological, psychological, social, and economic interactions, which interplay with external factors in the social and physical environment (Gewirtz \& Edleson, 2004; Manion, 2010; Mental Health Commission of Canada, 2012).

Exposure to IPV is an interactive factor that compromises mental health outcomes. Three decades of research have revealed that exposure to IPV negatively affects child development and functioning, and may lead to a multitude of mental health challenges (Bayarri, Ezpeleta, \& Granero, 2011; Bogat, DeJonghe, Levendosky, Davidson, \& von Eye, 2006; Gewirtz \& Edleson, 2004; Lamers-Winkelman, Willemen, \& Visser, 2012). The literature confirms that 35\% to 45\% of children who are exposed to IPV demonstrate clinically significant symptomatology (Bayarri et al., 2011). Correlational studies demonstrate that the average effect size for children's exposure to IPV and subsequent adjustment problems is -.29 (Kitzmann, Gaylord, Holt, \& Kenny, 2003). It is worth noting that the long-term mental health consequences for children who are exposed to violence are not significantly different from the outcomes for children who are directly victimized (Bayarri et al., 2011; Gewirtz \& Edleson, 2004; Lamers-Winkelman, Willemen, et al., 2012).

Children who witness IPV may present with a combination of significant mental, emotional, and behavioural impediments. Clinical challenges encompass everything from aggressive and non-compliant behaviours to emotional and internalizing problems; comorbidities across the range of symptoms are common (Bayarri et al., 2011; Gewirtz \& Edleson, 2004; Gewirtz \& Medhanie, 2008; Moylan et al., 2010). A meta-analysis of 118 studies on psychosocial variables by Meltzer et al. (2009) demonstrated that children who were exposed to IPV had significantly worse outcomes than those who were not so exposed. Similarly, Kitzmann et al. (2003) concluded that the results of their "current meta-analysis provided robust evidence that exposure to interparental aggression is associated with significant disruptions in children's psychosocial functioning” (p. 347). The effect size of this particular study indicates that approximately $63 \%$ of children who are exposed to IPV are faring more poorly and have ongoing functional challenges.

Using a developmental psychopathological perspective, Margolin and Gordis (2000) reviewed the effects of child maltreatment, community violence, and IPV at different developmental stages to determine how violence may compromise child development. Cited by 664 related articles in Google Scholar alone, this pivotal study exposed several domains of functioning that were negatively affected across the lifespan. The impacted developmental patterns that were identified included aggression and externalizing problems, depression and internalizing problems, psychobiological disturbances, ${ }^{6}$ PTSD symptomatology, delayed cognitive development and academic functioning, and difficulties in peer relationships.

Examining the available data, the authors state that the long-range effects of exposure to violence are clearly associated with mental health disorders and victimizing others. In conclusion,

\footnotetext{
${ }^{6}$ These disturbances included alterations in arousal, muscle tone, startle response, sleep disturbance, and abnormalities in cardiovascular regulation.
} 
International Journal of Child, Youth and Family Studies (2014) 5(4): 493-587

In Harm's Way: A Special Issue on the Impacts and Costs of Witnessing Intimate Partner Violence

Margolin and Gordis note that "outcomes associated with exposure to violence range in nature and severity" and, as the "child develops and matures, assessments of outcome must be viewed as highly time specific” (p. 469).

Examining multiple forms of victimization in a representative sample of 2,030 children, Turner, Finkelhor, and Ormrod (2006) determined that multiple forms of victimization in a child's life created an increased risk for mental health difficulties. Interestingly, Turner et al. noted that although forms of victimization may be interrelated, they demonstrated that "each victimization domain, including IPV, remained a significant predictor of mental health” (p. 13).

Employing longitudinal structural equation model tests on data gathered from the Lehigh Longitudinal Study - a prospective study of 457 youth and outcomes associated with family violence - Herrenkohl and Herrenkohl (2007) examined the impacts of physical and sexual abuse, child neglect, and IPV under the general constructs of child maltreatment. The children and their families who participated in the Lehigh Study were assessed three times: when they entered preschool (1976-77), when they were school aged (1980-1982), and when they were adolescents (1990-1992); 416 of these cases provided the basis for the Herrenkohl and Herrenkohl (2007) study. Accounting for the overlap of many possible factors in the general constructs of child maltreatment, Herrenkohl and Herrenkohl's results determined that child maltreatment independently predicted youth problems and lasting adverse effects, especially in the realm of externalizing and internalizing behaviours. Similarly, in a meta-analysis reviewing exposure to IPV and developmental consequences, Gewirtz and Edleson (2007) reported that children exposed to IPV exhibit more externalizing and internalizing behaviours, and that the impacts are chronic with lasting effects. Meltzer et al. (2009) reiterated these findings: Exposure to IPV can have serious effects on child development and well-being, including challenges with psychological adjustment and emotional and behavioural problems in adolescence. In fact, "the psychosocial outcomes of children witnessing domestic violence were not significantly different from those of physically abused children” (Meltzer et al., 2009, p. 492). Regrettably, many of these associated symptoms persist into adulthood.

Moylan et al. (2010) confirmed that children exposed to IPV had higher levels of externalizing and internalizing problems in adolescence. Testing for dual exposure effects, the authors determined that exposure to IPV and later outcomes are more complex than the literature would suggest. Similarly, in a longitudinal analysis of 1,816 caregivers and their children, Emery (2011) determined that there was a "robust relationship between IPV and externalizing and internalizing behavior” (p. 1553). Importantly, the researcher used a fixed-effects model to control for selection bias.

Following on the above, the majority of studies that focus on IPV and negative outcomes assess externalizing and/or internalizing mental health symptoms (Briggs-Gowan et al., 2010; Cosgrove et al., 2011; English et al., 2009; Ford, Gagnon, Connor, \& Pearson, 2011; Harding, Morelen, Thomassin, Bradbury, \& Shaffer, 2013; Hunter \& Graham-Bermann, 2013; Slade, 2007; Wright \& Fagan, 2012; Zarling et al., 2013), with much research documenting behavioural and emotional challenges in these two domains. A multitude of other researchers have reported similar findings with regard to externalizing and internalizing behavioural patterns and DSM-IV 
International Journal of Child, Youth and Family Studies (2014) 5(4): 493-587

In Harm's Way: A Special Issue on the Impacts and Costs of Witnessing Intimate Partner Violence

symptomatology (Jayasinghe, Jayawardena, \& Perera, 2009; Lamers-Winkelman, Willemen, et al., 2012; Lang \& Stover, 2008; Marmion \& Lundberg-Love, 2008; Meltzer et al., 2009; Moylan et al., 2010; Spilsbury et al., 2008).

It is also of note that gender differences with regard to emotional and behavioural problems related to IPV have been described (Bourassa, 2007; Camacho, Ehrensaft, \& Cohen, 2012; Helweg-Larsen, Frederiksen, \& Larsen, 2011; Spilsbury et al., 2007). Yet, some metaanalyses show no significant gender differences related to the psychological effects of witnessing IPV (Olaya, Ezpeleta, de la Osa, Granero, \& Doménech, 2010). The specific influences of gender remain unclear in the literature. As Spilsbury et al. (2007) state: "Collectively, research on gender differences in the externalizing vs. internalizing sequelae of exposure to domestic violence has produced disparate results” (p. 495). The research generally suggests caution with regard to relating certain psychological problems with specific genders; at the same time, the age-specific effects may be less difficult to determine in that the exposure at a younger age appears to be clearly associated with detrimental outcomes, with increasing age being correlated with fewer symptoms (Camacho et al., 2012; Helweg-Larsen et al., 2011; Spilsbury et al., 2007). Still, overall, the internalizing and externalizing symptoms of mental disorders are highly correlated with IPV; estimated factors range from .66 to .72 (Ford et al., 2011; Helweg-Larsen et al., 2011; Mikolajewski et al., 2013).

Children who have been exposed to IPV vary in their levels of social adjustment and in their resilience or coping mechanisms. When controlling for gender, ethnicity, age, and family environment variables, exposure to violence cumulatively over a child's lifetime accounts for more variance in adjustment than a child's age at first exposure (Graham-Bermann \& Perkins, 2010). Several other factors may also impact a child's adjustment. Owen, Thompson, Shaffer, Jackson, and Kaslow (2009) examined 129 low-income African-American children (ages 8 to 12) who had been exposed to IPV and the impact it had on their emotional and behavioural problems. The researchers found that exposure to IPV (i.e., experiencing or witnessing IPV) negatively impacted the families’ maternal psychopathology, family cohesion, and family relatedness quality (e.g., emotional quality or psychological proximity seeking), which were all associated with reduced child adjustment.

In an earlier study by Graham-Bermann, Gruber, Howell, and Girz (2009), the researchers explored how risk and protective factors, such as violence exposure and the individual characteristics of the mother, child, and family, impact a child's level of social and emotional adjustment and resilience to the adverse consequences of domestic violence. Their research suggests that the parental functioning level greatly impacts a child's adjustment or resilience. In their study, the researchers interviewed 219 mothers and their children (ages 6 to 12 ) in the community using standardized measures to assess different factors that may impact a child's adjustment (e.g., family violence, parenting, family functioning, maternal mental health, and children's adjustment and beliefs). Employing a cluster analysis, Graham-Bermann et al. (2009) discovered four different profiles of children's adjustment: children with severe adjustment problems (24\%); children who were struggling with adjustment (45\%); children who were depressed (11\%); and children who were resilient and had high levels of self-worth and competence and few adjustment problems (20\%). Children with severe adjustment problems 
International Journal of Child, Youth and Family Studies (2014) 5(4): 493-587

In Harm's Way: A Special Issue on the Impacts and Costs of Witnessing Intimate Partner Violence

witnessed more violence and their mothers had higher levels of depression and trauma symptoms, whereas the most depressed children were exposed to less violence but had higher levels of concern for their mothers' safety.

\section{Mediators in the Relationship Between IPV Exposure and Behaviour Problems}

Youths' relationships with their mothers or peers may mediate the effects of the domestic violence exposure (Camacho et al., 2012; Holt et al., 2008; Johnson \& Lieberman, 2007; Owen et al., 2008). Camacho and colleagues' (2012) study of 129 youth (aged 10 to 18) found significant associations between exposure to IPV resulting in injury and internalized behaviours $(\beta=.24, p<.05)$, but when female youths engage in, and are the recipients of, prosocial behaviours, they are at a decreased risk of developing internalized behaviours. These findings support the idea that "it is not the amount of peer support sought, rather, the perception of prosocial behavior received from peers, such as verbal kindness and expressions of caring, that predicts girls' reduced risk for developing internalizing problems” (Camacho et al., 2012, p. 136).

Another study by Owen et al. (2008) examined whether perceived social support for mothers and children exposed to domestic violence decreases emotional and behavioural problems for youth. With a sample of 148 low-income African-American children aged between 8 and 12, the researchers found that children's perceived social support provided a mediating role in the associations between IPV as reported by the child and internalizing and externalizing behaviour problems. On the other hand, the mother's perceived social support only played a mediating role between abuse and a child's internalized problems. However, Howell, GrahamBermann, Czyz, and Lilly (2010) also found that maternal factors had an impact on deleterious outcomes and resiliency in their study on children aged 4 to 6 who were exposed to domestic violence. In their sample of 56 mothers and children exposed to domestic violence within the previous 2 years, Howell et al. noted that "[b]etter parenting performance, fewer maternal mental health problems, and less severe or frequent violence exposure” predicted better prosocial and emotional regulation skills in youth (p. 158).

Mothers' attunement to children's emotional experiences, such as sadness and anger, also mediate the impact of exposure to domestic violence. Johnson and Lieberman's (2007) study of 30 preschool-aged children of mothers who had been domestically abused found that the children's externalizing behaviour was associated with the "severity of domestic violence prior to the marital separation, as well as with the current quality of the mother-child relationship and maternal attunement to the child's sad and angry feelings” (p. 304). The researchers found that mothers who were attuned to their children's feelings of sadness and anger, and were rated by a clinician to have a strong mother-child relationship, were better able to help prevent the onset of externalizing hyperactive or aggressive behaviour problems.

Still, children who are exposed to domestic violence have a higher risk of internalizing and externalizing problems than the non-exposed child population. Such problematic externalizing behaviour may include adjustment problems, delinquency, sexual behaviour problems, and interpersonal violence victimization or perpetration. Interventions which foster maternal attunement and social support must be tailored to reduce the impact on children 
International Journal of Child, Youth and Family Studies (2014) 5(4): 493-587

In Harm's Way: A Special Issue on the Impacts and Costs of Witnessing Intimate Partner Violence

exposed to domestic violence in order to ensure that they do not "slip through the net of fragmented services” (Buckley et al., 2007, p. 306), as the research is clear that such exposure increases adverse behaviour problems for youth.

Although many IPV studies focus on clusters of emotional and behavioural symptoms, as Slade (2007) notes, "much of our understanding of mental disorder descriptive epidemiology has been achieved by focused investigation of single mental disorders examined in isolation" (Slade, 2007, p. 554). As a result, a variety of studies that examine exposure to IPV and mental health outcomes review specific diagnoses and draw a variety of conclusions (Bayarri et al., 2011; Gewirtz \& Medhanie, 2008; Hungerford, Wait, Fritz, \& Clements, 2012; Moylan et al., 2010). Mental illnesses that have been investigated in relation to IPV include PTSD, anxiety, and depression. This literature is reviewed next.

Post-traumatic stress disorder and anxiety disorders. As emphasized throughout this review, the literature makes clear that each child is unique, and the distress caused by exposure to IPV within the home can be expressed differently even among siblings (Horn, Hunter, \& Graham-Bermann, 2013). For some youth, distress from IPV may manifest in post-traumatic stress disorder (PTSD). PTSD is a normal stress reaction to abnormal events that involve the threat of injury or death (American Psychiatric Association, 2013). In studying preschool trauma associated with IPV, Bogat et al. (2006) noted that children aged 3 to 12 manifest trauma symptoms as a result of witnessing IPV. Bogat and colleagues' study further showed that nearly half of the participants exhibited at least one trauma symptom following exposure to family violence. Examining a clinical sample, Crusto et al. (2010) arrived at similar conclusions: PTSD was positively and significantly correlated with family violence; the risks increased with the number and types of trauma experienced, and led to detrimental mental health outcomes in adulthood (Crusto et al., 2010). In the United States, research suggests that approximately 50\% of children exposed to IPV between the ages of 1 and 7 years old experience trauma symptoms, with increased frequency of exposure associated with more PTSD symptoms (Levendosky, Bogat, \& Martinez-Torteya, 2013). As well, Lamers-Winkelman, Willemen, and Visser (2012) found rates similar to those in the United States in their high-risk Dutch sample, where between $45 \%$ and $54 \%$ of children exposed to IPV demonstrated high levels of trauma.

In a meta-analysis of IPV exposure studies, Evans et al. (2008) found a significant association between IPV exposure and trauma symptoms (estimated $d$-value of 1.54). Other researchers have recognized PTSD as a pathological outcome of exposure to IPV. Olaya et al. (2010) assessed 520 children aged between 8 and 17 years and showed that living with IPV increased the risk of PTSD; Spilsbury et al. (2007) discovered clinically significant levels of several trauma symptoms related to IPV exposure; and Meltzer et al. (2009) also noted the higher risk of developing PTSD with measured IPV in the home. Additionally, the post-traumatic stress symptoms that arise as a result of IPV exposure may also mediate internalizing and externalizing behaviour problems that may not be direct effects of IPV exposure, which suggests a comorbidity between traumatic stress and child adjustment (Miller, Howell, \& Graham-Bermann, 2012). As well, Cisler et al. (2012) have demonstrated that cumulative IPV exposure predicts subsequent PTSD, depression, delinquency, and binge drinking $(\beta \mathrm{s}=.07, .12, .10$, and .09, respectively, $p<.01$ in all cases). 
International Journal of Child, Youth and Family Studies (2014) 5(4): 493-587

In Harm's Way: A Special Issue on the Impacts and Costs of Witnessing Intimate Partner Violence

Children who experience trauma from IPV may also be exposed to additional traumatic events (e.g., sexual assaults, physical assaults, serious illnesses) that can aggravate traumatic stress symptoms. As Graham-Berman, Castor, Miller, and Howell (2012) found in their study of 120 children, aged 4 to 6 years, who had been exposed to IPV within the previous 24 months, at least $38 \%$ of the children were exposed to other traumatic events in addition to IPV. In comparison to children exposed solely to IPV, these children exposed to multiple stressors (IPV and other traumatic events) had a greater likelihood of PTSD diagnoses, more traumatic stress symptoms $(d=0.96)$, and more internalizing $(d=0.86)$ and externalizing behaviour $(d=0.47)$ problems.

PTSD does not disappear after childhood and can differ in severity depending on the other factors present during childhood and IPV exposure (Anderson \& Bang, 2012). As Anderson and Bang (2012) found, adult women who experienced police intervention in relation to childhood IPV incidents had higher PTSD rates than women who did not experience police interventions $(t=-2.90, p=0.005)$. Similarly, adults who reported that they were exposed to IPV and had mothers with mental health problems also reported higher PTSD rates and than those whose mothers did not $(t=-2.95, p=0.005)$. Although less studied than PTSD, longitudinal analyses have examined the relationships between witnessing IPV and anxiety symptomatology. Anxiety is a general term for several disorders that contribute to nervousness, fear, apprehension, and excessive worrying. Anxiety disorders can be extremely debilitating and can have ongoing detrimental impacts (American Psychiatric Association, 2013). Studies reveal that witnessing IPV is intricately connected to anxiety disorders and, compounding the problem, that there is an associated risk of exposure to community violence for affected youth (Bourassa, 2007; BriggsGowan et al., 2010; Kennedy, Bybee, Sullivan, \& Greeson, 2009; Slade, 2007). These papers, and others, provide conclusive evidence of the significant relationships between exposure to IPV and resultant PTSD and anxiety disorders.

Also linked to IPV, but to date not as extensively investigated as some other mental illnesses, is depression, which as the American Psychiatric Association (2013) states is often chronic and follows a pattern of remission and relapse that affects individuals' adaptive functioning. Studies that have examined the effect of IPV exposure in relation to depression have thus far yielded mixed results. Employing a longitudinal, multi-information design, Sternberg, Lamb, Guterman, and Abbott (2006) found that there was "little stability over time in the pattern of reported effects, and children were more likely than other informants to report levels of maladjustment that varied depending on recent or concurrent exposure to family violence” (p. 283). Information obtained from mothers, fathers, children, and teachers provided a range of results with little agreement between groups. The findings did suggest that with regard to depression, younger children (under age 10) were more susceptible to the effects of IPV. Yet, some of the children who had problems in the early years did not have challenges later. The authors determined that the chronicity, severity, and nature of IPV likely influenced the results (Sternberg, Lamb, et al., 2006).

Kennedy, Bybee, Sullivan, and Greeson (2010) conducted a longitudinal study to examine the relationships between several types of violence exposure including IPV, and 
International Journal of Child, Youth and Family Studies (2014) 5(4): 493-587

In Harm's Way: A Special Issue on the Impacts and Costs of Witnessing Intimate Partner Violence

depression among 100 school-aged children. The authors found significant between-child differences in depression, both initially and over the course of the study, notwithstanding the risks of IPV. Like Sternberg, Lamb et al. (2006), Kennedy et al., (2010) posit that youth respond differently to IPV due to a number of factors. On the other hand, Kennedy et al. did find that a reduction in IPV exposure was significantly associated with lower levels of depression over time. Kennedy et al. also noted that initial support and gender were moderating variables; that is, boys' levels of depression did not significantly decline with reductions in IPV exposure, while support appears to have contributed to a decline in depression for girls.

Additionally, several small scale and population-based studies conducted since 2009 have revealed correlations between IPV exposure and depression among adolescents and across the entire lifespan (Nguyen \& Larsen, 2012; Roustit et al., 2009; Slopen, Fitzmaurice, Williams, \& Gilman, 2012). Perhaps Bayarri and colleagues (2011) draw the most prudent conclusion given the current knowledge base: "all children and adolescents, regardless of their age and sex, and irrespective of their level of exposure to IPV, are equally at risk of experiencing psychological problems” (p. 542).

Thus far, we have discussed the internalizing manifestations of these problems. We follow next with externalizing manifestations.

\section{Attention Problems}

Exposure to IPV is associated with the development of attention and conduct problems for youth. Becker and McCloskey (2002) used a two-part longitudinal study (Time $1=$ 1990/1991, Time $2=1996 / 1997)$ to examine the impact of IPV on youth. At Time 1 , mothers ( $n$ = 287) were interviewed using the Child Assessment Schedule (CAS) to assess attention deficit/hyperactivity disorder and conduct disorder in one of their children (aged 6 to 12); children were interviewed at Time 2. The study's findings suggest that domestic violence is a strong predictor of attention problems and conduct problems for girls, but it did not necessarily lead to delinquency.

Although many researchers have examined externalizing symptoms concerning IPV exposure, few studies have discretely examined specific externalizing disorders in relation to IPV exposure. The results that have been extrapolated offer some concerning correlations. Meltzer at al. (2009) revealed that, "witnessing severe domestic violence almost tripled the likelihood of children having conduct disorder but was not independently associated with emotional disorders” (p. 491). Briggs-Gowan et al. (2010) also confirmed that witnessing IPV was associated with conduct problems and the disruptive behaviours of attention deficit/ hyperactivity disorder (ADHD) in older children. However, although there is a paucity of research examining ADHD and IPV exposure, preliminary studies of ADHD and maltreated children indicate that there may be significant problems associated with IPV exposure (BeckerBlease \& Freyd, 2008; Olaya et al., 2010). This association certainly becomes clear in a recent prospective American study (Bauer, Gilbert, Carroll, \& Downs, 2013) that examined 2,422 children in the United States and found that, even when controlling for sex and ethnicity, children of parents who self-reported experiencing both IPV and depressive symptoms between 
International Journal of Child, Youth and Family Studies (2014) 5(4): 493-587

In Harm's Way: A Special Issue on the Impacts and Costs of Witnessing Intimate Partner Violence

the child's birth and 2 years of age had increased odds of being diagnosed with ADHD between ages 3 and 6 (adjusted OR = 4.0, 95\% CI, 1.5-10.9).

\section{Conduct and Behavioural Problems}

Behavioural problems are prevalent among children who experience all forms of domestic violence (English et al., 2009). However, children who are exposed to both physical and psychological IPV are at higher risk of behaviour and conduct problems than children with no exposure, even though they may not necessarily experience child abuse themselves (Renner, 2012). For instance, one study found that the 2- to 3-year-old children of mothers victimized by IPV were, respectively, 2.72 and 3.48 times more likely to surpass the clinical cut-off for externalizing behaviour than children who had not been exposed to IPV (DeJonghe, von Eye, Bogat, \& Levendosky, 2011). Such associations between IPV exposure and negative outcomes persist into a child's adolescence and adulthood (Ehrensaft \& Cohen, 2012; Herrenkohl \& Herrenkohl, 2007; Holt et al., 2008; Israel \& Stover, 2009; Jarvis, Gordon, \& Novaco, 2005; Moylan et al., 2010). In fact, according to Meltzer et al. (2009), children who have witnessed severe incidents of domestic violence are almost three times as likely to develop conduct disorders (OR $=1.78,95 \% \mathrm{CI}=1.79-3.92, p<0.001$, Meltzer et al., 2009). Similarly, Becker and McCloskey's (2002) longitudinal study on attention and conduct problems for youth exposed to domestic violence found that girls exposed to domestic violence experienced attention problems related to concurrent conduct problems.

The evidence for concurrence and for variations in that concurrence mounts: In a study by Spilsbury et al. (2008), researchers examined adjustment problems in a community-programbased sample consisting of 175 school-aged children who had been exposed to domestic violence. These researchers found that $18 \%$ of the sample demonstrated externalizing problems (both with and without additional internalizing problems); $11 \%$ of the sample demonstrated both externalizing and internalizing problems, whereas only $13 \%$ met the clinical threshold for internalizing problems. Further, domestic violence exposure may co-occur in other negative environmental settings, such as child abuse, community violence, and parental alcohol abuse, which all influence negative externalizing behaviour (Herrenkohl et al., 2008; Malik, 2008; Moylan et al., 2010; Ritter, Stewart, Bernet, Coe, \& Brown, 2002). For example, Ritter and colleagues (2002) found that exposure to domestic violence and parental alcohol abuse increased the risk for delinquency and conduct disorders for young females, and such environments negatively affected both male and female youths’ self-esteem.

Animal cruelty may be another adverse consequence from a child's exposure to domestic violence. Duncan, Thomas, and Miller (2005) completed 289 chart reviews of young boys in residential treatment to examine the differences between children (aged 8 to 17 years) who had conduct behaviour problems or conduct behaviour problems and a history of cruelty to animals. The researchers found that when comparing young boys with conduct disorders only $(n=50)$ to the boys who had both conduct disorders and recorded incidents of animal cruelty $(n=50)$, the latter group had significantly greater histories of physical child abuse $(p=.036)$, sexual abuse $(p$ $=.048)$, and exposure to domestic violence $(p=.050)$. The study authors concluded that the 
International Journal of Child, Youth and Family Studies (2014) 5(4): 493-587

In Harm's Way: A Special Issue on the Impacts and Costs of Witnessing Intimate Partner Violence

male youth exposed to domestic violence were almost twice as likely as the comparison group to engage in animal cruelty.

\section{Substance Misuse}

The problematic impact of young people's exposure to IPV is also strongly felt in another externalizing behaviour: substance misuse. Childhood maltreatment in all its forms, including witnessing IPV (Butchart, Harvey, Mian, \& Furniss, 2006), has been identified as a key contributing factor to substance misuse in adolescents and adults. Widom, Marmorstein, and White (2006), in their prospective study on childhood victimization and illicit drug use in middle adulthood, found that at least 20 studies published between 1975 and 1989 demonstrated evidence for a relationship between childhood abuse and substance use. As Hovdestad, Tonmyr, Wekerle, and Thornton (2011) state, “childhood maltreatment and adolescent substance abuse are important health issues that have been linked by research and theory for at least 50 years" (p. 525). However, discerning the exact contribution of witnessing IPV to adolescent substance misuse is a difficult task, firstly, because genetic influences may play a role in substance use, and secondly, because substance use (not misuse) is generally well distributed among adults and adolescents.

With regard to genetic influences, Enoch (2011), in her extensive and thorough review of more that 190 studies that focus on genetic and environmental influences on the development of alcohol dependence, states quite definitively that, "experiencing maltreatment and cumulative stress prior to puberty and particularly in the first few years of early life is associated with early onset of problem drinking in early adulthood" (p. 17). She further states that research has shown that "environmental stressors can swamp genetic influences" (p. 17). The negative impact of exposure to abuse and neglect on development, especially neurological development, has already been examined in an earlier section of this review. Nonetheless, it is highlighted again here in order to underline the reach of this negative impact.

With regard to the second issue, typical and atypical substance use, Hovdestad et al. (2011), state that, "substance use and abuse among adolescents must be distinguished because it is considered normative that the majority of adolescents experiment with alcohol and many experiment with other substances” (p. 3). We concur and took care to make sure that this distinction was clearly described and acknowledged in the literature included in this review.

Non-typical use of substances. Citing data gathered in Canada (Canadian Alcohol and Drug Use Monitoring Survey [CADUMS], 2009) and the Centers for Disease Control and Prevention (2008) in the United States, Hovdestad et al. (2011) note that in representative samples from both countries of youth aged 12 to 17 years, about 53\% reported using alcohol and just over $27 \%$ reported using cannabis in the previous year. Abuse of alcohol and other substances therefore needs to be distinguished from these normative levels. Alcohol abuse is defined by Hovdestad et al. and others (e.g., Centers for Disease Control and Prevention, 2012; Hamburger, Leeb, \& Swahn, 2008) as binge or heavy episodic drinking (five or more drinks per occasion) at least once per month or more. According to Clark, Bukstein, and Cornelius (2002), approximately 33\% of adolescents in the United States engage in such binge drinking with about 
International Journal of Child, Youth and Family Studies (2014) 5(4): 493-587 In Harm's Way: A Special Issue on the Impacts and Costs of Witnessing Intimate Partner Violence

6\% progressing to alcohol use disorders. In Canada, approximately $28 \%$ of those surveyed by CADUMS (2009) reported binge drinking at least once per month or more (as cited in Hovdestad et al., 2011).

Using data gathered via the Ontario Student Drug Use and Health Survey, Paglia-Boak, Mann, Adlaf, and Rehm (2009) show that among Canadian students aged 15 to 24, 25.5\% had used cannabis at least once in the past three months, with $16 \%$ of the identified $25.5 \%$ reporting monthly use, $29.1 \%$ reporting weekly use, and $18.4 \%$ reporting daily use. It is not clear from these reports how many students also became dependent or sought treatment for problematic cannabis use. United States data cited by Hovdestad et al. (2011) do, however, give us some indication: Using American treatment program admission data as the basis for their estimates, these researchers state that " $16 \%$ of all admissions reported that marijuana [cannabis] was their primary substance and of those 16\%, 41\% were adolescents” (p. 528). Simple arithmetic therefore suggests that, as with alcohol misuse, approximately $6 \%$ to $6.5 \%$ of adolescent cannabis users become cannabis misusers (Substance Abuse and Mental Health Services Administration [SAMHSA], 2007 as cited in Hovdestad et al., 2011).

Alcohol and cannabis are, of course, not the only substances that are misused and, increasingly, research on the abuse of substances is moving away from single substance studies to polysubstance use and misuse studies (Kendler et al., 2012; Schensul, Convey, \& Burkholder, 2005). However, despite the rising awareness of polysubstance use and misuse, alcohol and cannabis are still the most common substances used by adolescents and therefore these substances still receive the most research attention. Although the negative and harmful impacts of the misuse of these substances on the lives of adolescents are also well studied, this research is beyond the scope of this literature review. However, it is important to note that it is well recognized that substance misuse is linked with a plethora of negative outcomes for adolescents, so much so that in May of 1998, the United States Department of Justice Office of Juvenile Justice and Delinquency Prevention published an online research summary of the consequences of youth substance abuse (US Department of Justice Office of Juvenile Justice and Delinquency Prevention, 1998). This summary shows that youth substance abuse is linked to poor grades, higher truancy and higher levels of dropping out of school, poor socioeconomic outcomes, higher levels of delinquency and higher crime rates, poor physical health, higher levels of car accidents, physical disabilities and disease including HIV/AIDS, and significantly greater risks of death from accidents, illness, suicide, and homicide (US Department of Justice Office of Juvenile Justice and Delinquency Prevention, 1998). In light of this research, it is extremely important that we fully understand how substance misuse develops in young people.

As noted above, it is well documented that child maltreatment plays a major role in the development of substance misuse. However, not all research that has been conducted on the link between child maltreatment and substance misuse necessarily includes witnessing IPV as a part of such maltreatment. Along with disentangling typical substance use from atypical substance use, unravelling the impact of witnessing IPV from other forms of child maltreatment provides an additional challenge. Still, since 2008, the United States Centers for Disease Control Prevention Report has included witnessing IPV in the definition of child maltreatment (Leeb, Paulozzi, Melanson, Simon, \& Arias, 2008) and the literature on child maltreatment has begun to 
International Journal of Child, Youth and Family Studies (2014) 5(4): 493-587

In Harm's Way: A Special Issue on the Impacts and Costs of Witnessing Intimate Partner Violence

pay greater attention to this form of maltreatment. It is therefore more likely that studies conducted after 2008 will include mention of witnessing IPV in definitions of maltreatment and will more readily assist us in assessing the impact of IPV exposure on children and youth. In this examination of the literature on the links between child maltreatment and substance misuse, we scrutinized the published research generated by our search and selected only studies that included witnessing IPV as a form of child maltreatment or which focused explicitly on the relationship between witnessing IPV and substance misuse.

As we conducted this review, we also paid careful attention to method and followed the well-supported suggestion given by Gilbert et al. (2009) who underline the importance of longitudinal and prospective research to understanding relationship consequences of child maltreatment (including witnessing IPV) to the development of substance misuse issues in children and youth. These researchers state:

Since we are interested in the consequences of child maltreatment we want to assess causality. Thus the strengths of prospective studies include the temporal ordering of maltreatment and subsequent outcomes, objective measurement of maltreatment, avoidance of recall bias, minimization of selective inclusion of participants on the basis of outcome and the opportunity to adjust for social and individual confounding factors as they occur. (p. 74)

Longitudinal and prospective research. Summarizing the longitudinal and prospective research on IPV exposure and its impact on the development of substance misuse is challenging despite the fact that only four studies were published within the time frame of interest for this review. Each of these four studies had somewhat different research questions, and each used different measures, different age groups with different socioeconomic, cultural and ethnic locations, different sample sizes, and different methods for collecting data. Thus, as cross-study comparisons are difficult, the better approach may be to examine the convergence and divergence across studies and across the timelines within which these studies were conducted.

Fergusson, Boden, and Horwood's (2008a) 25-year, prospective, longitudinal study of the developmental antecedents of illicit drug use was conducted in New Zealand and followed a cohort of 1,265 children (635 males, 630 females) born in Christchurch in 1977. These children were studied at birth, 4 months, and 1 year of age, and at ages $6,18,21$, and 25 . The focus of the study was the use, abuse, and dependence on "other illicit drugs" (i.e., not alcohol or cannabis). Other illicit drugs were defined as:

solvents (glue, petrol, and paint); stimulants (including methamphetamine); barbiturates; other prescription medications that were illicitly obtained; opiates, including both heroin and morphine; cocaine (in any form); hallucinogens including ecstasy, LSD, and PCP; and any other substances (primarily plant extracts) including mushrooms and datura. (p. 168)

The study involved a variety of measures from a number of sources: parent surveys and interviews, teachers' reports, cohort members' self-reports, medical and other records, and 
International Journal of Child, Youth and Family Studies (2014) 5(4): 493-587

In Harm's Way: A Special Issue on the Impacts and Costs of Witnessing Intimate Partner Violence

psychometric assessments. Multiple contributing factors for illicit drug use known to be implicated in 1977, when the study was first conceptualized, were examined, including personal characteristics (e.g., child's gender, childhood adolescent adjustment, child novelty seeking, affiliation with substance-using peers), parental adjustment factors (e.g., parental illicit drug use, parental criminality, parental alcohol problems, parental use of physical punishment and physical abuse, interparental violence), and cohort members' use of cigarettes and alcohol at ages 16 and 25.

One problematic aspect of Fergusson et al.'s (2008a) study is that, although IPV among the parents of child and youth cohort members was investigated, this was not done until cohort members reached the age of 18. Only at this point were youth participants, but no one else, asked to retrospectively report on their exposure to IPV prior to the age of 16. The questions asked of the 18-year-olds were based on 8 items derived from the Conflict Tactics Scale or CTS (Straus, 1979) and its follow-up, the revised CTS2 (Straus, Hamby, Boney-McCoy, \& Sugarman, 1996), a scale that is still frequently used to assess psychological and physiological attacks on intimate partners. Interestingly, although the CTS is designed specifically for assessing IPV in adult partners, in Fergusson et al.'s (2008a) study, only participating 18-year-old youth were asked to report on this issue. The omission of parental self-report data about IPV that could well have been collected during each wave of the longitudinal study seems curious, especially because Fergusson et al. used the CTS to question youth and seemed to have recognized, from the start, the potential impact on the cohort members of witnessing IPV. Still, Fergusson et al., using regression analysis, did detect a significant association between youth cohort members' illicit drug use, abuse, and dependence at ages 16 to 25 for both males and females $(p<.0001)$, at a level of association that was the same as that found for exposure to sexual abuse in childhood (both queried retrospectively prior to the age of 16, only of youth cohort members at ages 18 and 21), for novelty seeking assessed via self-reports at age 16, and for conduct problems which had been assessed via teacher reports at ages 7 and 13. All these measures yielded a higher level of significance $(p<.0001)$, than that detected for exposure to physical punishment $(p<.001)$, which was also examined retrospectively for the time period prior to the age of 16, only of youth cohort members at ages 18 and 21. Finally, gender (that is sex, since no definition of gender was provided) also emerged as significant in that males were found to use, abuse, and be dependent on illicit drugs at significantly higher levels than females $(p<.01)$.

Interestingly, the significant association for witnessing IPV prior to the age of 16 did not hold when Fergusson et al. (2008a) conducted a multivariate analysis of all the risk factors that they included in their study in order to establish the time dynamic significance of these factors. The multivariate analysis found that with regard to the three abuse exposure factors (IPV, childhood physical abuse, childhood sexual abuse), only childhood sexual abuse $(p<.0001)$ remained significant. Being male $(p<.0001)$, novelty seeking $(p<.0001)$, and conduct problems between the ages of 7 to $13(p<.05)$ also remained significant, but the scores for novelty seeking and conduct problems may well have been influenced by the timing of the various measures used in the Fergusson et al. study. We note that participants' childhood conduct and attention problems were examined during the time that cohort members were aged 7 to 13 , but their exposure to IPV, childhood sexual abuse, and childhood physical abuse were not investigated until after their conduct problems had been measured and already established as 
International Journal of Child, Youth and Family Studies (2014) 5(4): 493-587 In Harm's Way: A Special Issue on the Impacts and Costs of Witnessing Intimate Partner Violence

implicated in the development of the use of illicit drugs. Given the distinct possibility that conduct and attention problems can be triggered and exacerbated by witnessing IPV (Becker \& McCloskey, 2002; English et al., 2009; Meltzer et al., 2009), by not investigating exposure to IPV until cohort members were 18 years of age and then only through retrospective reports, it is quite possible that the impact of cohort members' witnessing IPV on the development of their conduct problems was missed, while at the same time, their conduct problems were foregrounded as predictive of the development of their illicit drug use, abuse, and dependence at ages 18 to 25. It is conceivable that these conduct problems may well have developed, at least in some significant part, because participants had been witnessing IPV before the age of 7 .

To understand the pre-age 7 effects of exposure to IPV we turn to Andersen and Teicher (2009), who conducted an extensive review of the recently reported effects of early stress on brain development in order to examine the causal links between such developmental stress exposure and subsequent risk for substance abuse. In the abstract of their review, Andersen and Teicher state that the "interaction of exposure [to childhood adversity] during a sensitive period and maturational events produces a cascade [italics ours] that leads to the initiation of substance use at younger ages, and increases the likelihood of addiction by adolescence or early adulthood" (p. 516). Their definition of childhood adversity includes exposure to witnessing domestic violence and they place this stress on par with abuse, parental loss, and household dysfunction, all of which they identify as implicated in producing "a cascade of physiological and neurohumoral events that alter trajectories of brain development” (p. 517) such that this altered development creates a significant vulnerability for drug abuse. Andersen and Teicher describe drug abuse as a developmental disorder and offer compelling evidence for their claims by reviewing the findings of more than 190 studies relevant to the understanding of the impact of early life exposure to adversity on brain development published between 1978 and 2007. While exposure to IPV was not the only adversity examined in this review, it is included among the kinds of stressors that significantly increase the potential for changes in the hypothalamicpituitary-adrenal axis, such that those who are affected experience what Andersen and Teicher describe as a "revved up" reward system that predisposes people to the compulsive use of substances that manifests at an early age.

Smith, Elwyn, Ireland, and Thornberry (2010) also refer to a cascading effect, although the cascade that they refer to is not physiological and neurohumoral; their cascade is made up of negative consequences. Smith et al., who examined the impact of adolescent exposure on substance use in early adulthood in their longitudinal Rochester Youth Development Study (RYDS) beginning in 1987, propose that, "exposure to family violence along with other family processes potentially contributes to a cascading series of consequences that lead from short-term reactive responses to entrenched longer term consequences such as drug and alcohol problems" (p. 220). They examine this proposition in a subset of 508 participants (77\% male, 23\% female; 58\% African-American, 18\% Latino, and 22\% White) who were enrolled in the RYDS, which involved 1,000 mostly urban, high-risk, minority adolescents who were already seen as extremely vulnerable for engagement in delinquency and crime. The 508 selected participants in the subsample were those whose primary caretakers were either married or had cohabiting partners in at least one of the waves of data collection during which partner violence was assessed with the CTS (Straus, 1979). As with many samples of youth who are at risk for 
International Journal of Child, Youth and Family Studies (2014) 5(4): 493-587

In Harm's Way: A Special Issue on the Impacts and Costs of Witnessing Intimate Partner Violence

delinquency and crime, there were considerably more males in the group than there were females (Chesney-Lind \& Jones, 2010; Zahn, 2009). Smith et al. found that the participants reported considerable substance use: 56\% of males and 33.9\% of females reported any drug use, with $19.7 \%$ of males and $8 \%$ of females reporting drug use problems; as well, $42.2 \%$ of males and $17.4 \%$ of females reported heavy drinking, with $32.5 \%$ of males and $16.1 \%$ of females reporting any alcohol abuse problems.

The self-reported levels of drug abuse and problem drinking in Smith et al.'s (2010) highrisk population were certainly higher than the levels reported for the general adolescent population in the United States where approximately 33\% of adolescents were shown to engage in binge drinking with about 6\% progressing to alcohol use disorders (Clark et al., 2002) and about $6 \%$ to $6.5 \%$ of adolescent cannabis users becoming misusers, as noted earlier in this section. However, in Smith et al.'s (2010) population sample, exposure to severe IPV was found to increase the odds of alcohol problems (not other substances) in early adulthood only for young women $(\mathrm{OR}=5.63, p<.05)$. While these results raise awareness of the greater potential for alcohol abuse among high-risk, marginalized girls who are exposed to IPV, these findings cannot be treated as informative for a general population, even if restricted to females. The females in this study belong to a highly select group: high-risk girls. Although Smith et al.'s study is longitudinal and the data-gathering tool was standardized, the participants in the study comprise a very particular marginalized minority, high-risk, predominantly male group that cannot be seen as representative of a more general population.

Greater generalizable weight may be given to the findings of Skeer, McCormick, Normand, Buka, and Gillman (2009), who utilized data from a three-wave longitudinal study (Project on Human Development in Chicago Neighborhoods) conducted between 1994 and 2001 in multiple neighbourhoods in Chicago, Illinois, rather than a single high-risk location. Skeer et al.'s data were drawn from a stratified probability sample of 80 neighbourhoods selected from 343 neighbourhood clusters, from which 20-block groups were selected at random from each of the 80 neighbourhoods. To select the 1,421 children aged 10 to 22 (51\% female; $44 \%$ Hispanic/Latino, 37.4\% Black, 14\% White) who were the focus of the substance disorder study, the researchers drew a systematic random sample of residences from the sampled block groups and screened all the households in each block group ( 40,000 households), which they enumerated and screened for the presence of age-eligible children. They subsequently included those children aged between 10 and 16 at enrolment in their study on the development of substance disorders in adolescence. In this more generalizable group, family conflict was assessed in Wave I (1994 to 1997) at baseline using Moos and Moos' (1986) standardized Family Environment Scale (FES), which examines nine behaviours (e.g., family members hitting each other, being openly angry, losing their tempers), when the child participants were between 10 and 16 years of age. Psychological stress was measured at Wave II (1997 to 1999) using two standardized scales, Youth Self Report or YSR (Achenbach, 1991) for internalizing problems and the Child Behaviour Checklist or CBCL (Achenbach \& Edelbrock, 1983) for externalizing problems, when participants were from 13 to 19 years of age. Substance use disorders (SUD) were assessed at Wave III (2000 to 2001), when participants were between 16 and 22 years of age, using the DSM-IV diagnosis for alcohol dependence or marijuana abuse or dependence. At Wave I, data were also gathered on external social supports from immediate family, extended 
International Journal of Child, Youth and Family Studies (2014) 5(4): 493-587

In Harm's Way: A Special Issue on the Impacts and Costs of Witnessing Intimate Partner Violence

family, friends or neighbours, school or community members, or others to see if these supports buffered against familial conflict. Also at Wave I, additional confounding factors known to be associated with both familial conflict and substance disorders were examined: parental history of substance abuse, parental history of depression, primary caregiver marital status, family socioeconomic status, and exposure of children to physical abuse and/or punishment.

Using bivariate and multiple logistic regression models to evaluate the association between familial conflict at Wave I with adolescent substance use disorders at Wave III, Skeer et al. (2009) showed the following:

In the bivariate analyses, familial conflict at Wave I was significantly associated with SUDs in Wave III, indicating that a one standard deviation increase in familial conflict was associated with a 24\% higher odds of a substance use disorder (OR: 1.24; 95\% CI: 1.05-1.46). Additionally, males were more likely to have an SUD at Wave III (OR: 1.61; CI: 1.19-2.18), as were individuals whose parents had a history of substance use problems (OR: 1.46; CI: 1.01-2.11) [...] In the fully adjusted logistic regression model, the association between familial conflict and substance use disorders was unchanged (OR $=1.23$; $\mathrm{CI}=1.02,1.47)$. Other significant covariates in the adjusted model were gender and family SES [...] Finally, we added the interaction term between familial conflict and social support to the adjusted model to test the buffering hypothesis described above; however, it was not significant ( $p=0.815)$. (p. 69)

In referring to their primary hypothesis that "familial conflict during childhood will increase the risk of developing a substance use disorder during adolescence” (Skeer et al., 2009, p. 66), they state:

Our primary hypothesis was substantiated in this study, as evidenced by our finding that controlling for a number of demographic and parental and family characteristics, children who lived in families with higher levels of familial conflict when they were younger had a significantly greater risk for developing substance use disorders in late adolescence and emerging adulthood. This is important because not only is it consistent with other studies examining this association (Rhodes \& Jason, 1990; Vakalahi, 2001), this is one of the only studies to examine this relationship using longitudinal data, thereby avoiding the alternative reverse causation hypotheses. Furthermore, we found that almost one-third of the cases of substance use disorders in the sample would have been prevented if the adolescents had not lived in family environments with elevated levels of familial conflict. This underscores the need for prevention efforts to focus on adversity in the home. (p. 70)

Certainly the evidence presented by Skeer et al. (2009) is persuasive and at the same time, where gender is concerned, contradicts the evidence presented by Smith et al. (2010). To recap, Smith et al. found that exposure to severe IPV increased the odds of alcohol problems (not other substances) in early adulthood only for the young women in the RYDS. But Skeer et al. found a significant relationship between exposure to familial conflict and substance disorders for both females and males, with males being even more likely than females to develop substance use disorders when exposed to familial conflict. On careful examination of both studies, we suggest 
International Journal of Child, Youth and Family Studies (2014) 5(4): 493-587

In Harm's Way: A Special Issue on the Impacts and Costs of Witnessing Intimate Partner Violence

that the Smith et al. study carries less overall weight. The Smith et al. study is based on a highly select subsample from the RYDS and that calls into question the generalizability of the findings. We have greater confidence for generalizability in Skeer et al. (2009), given their more extensive sampling procedures as described above.

Further longitudinal research that supports a negative relationship between witnessing domestic violence (family-based adversity) and substance misuse was also provided by the fourth and final longitudinal study we were able to locate: Begle et al. (2011) used a nationally representative sample of 3,614 adolescents aged 12 to 17 drawn from a United States national household probability sample (2005 National Survey of Adolescents [NSA-Replication]), to examine "the direction of the association between victimization and high risk behavior and the role of gender” (p. 683). These researchers hypothesized that victimization experiences are predictive of engagement in high-risk behaviour. The victimization variables that they investigated included physical abuse and/or assault, sexual abuse, and witnessed community and domestic violence. The high-risk behaviours examined included alcohol use, drug use, and delinquency. The data were gathered through highly structured telephone interviews with the focus in Wave I being on establishing a lifetime prevalence baseline for victimization along with year one information about all the behavioural variables in question. Wave I and Wave II data collection interviews were conducted approximately one year apart. Witnessed domestic violence was described as participants having observed their parents punch, hit, or beat up one another, choke one another, hit one another with an object, or threaten one another with a gun, knife, or other weapon. Data were analyzed using structural equation modelling and showed that, as hypothesized, victimization at Wave I significantly predicted high-risk behaviour incidence for both males and females at Wave II. Unfortunately, the multiple and separate victimization constructs, and the multiple and separate high-risk behaviour constructs, were combined to create a single composite victimization variable and a single composite high-risk behaviour variable for the purposes of analysis. This means the model cannot be teased apart in order to ascertain the contribution of witnessed domestic violence to alcohol and drug use and abuse. However, Begle and colleagues did report that:

The baseline model for victimization provided a good fit to the sample data, $\chi 2(15, N=3614)=812.39, p<0.001, \mathrm{NFI}=0.94, \mathrm{CFI}=0.94, \mathrm{SRMR}=0.05$. Victimization at Wave 1 significantly predicted victimization at Wave $2(\beta=0.68, B=$ 22.76, $p<0.05$ ) suggesting invariance of the construct over time. Similarly, the baseline model for high risk behavior provided an adequate fit, $\chi 2(29, N=3614)=4253.85, p<$ 0.001 , NFI $=0.92$, CFI $=0.92, \mathrm{SRMR}=0.08$. High risk behavior at Wave 1 significantly predicted behavior at Wave $2(\beta=0.73, B=43.88, p<0.05)$. (pp. 685-686)

Additionally, Begle et al. (2011) reported the following:

Results indicated that the model combining these constructs provided adequate fit to the hypothesized model, $\chi 2(122, N=3614)=8198.33, p<0.001$, NFI $=0.90$, CFI $=0.91$, $\mathrm{SRMR}=0.10$. Investigation of individual paths revealed that victimization at Wave 1 significantly predicted high risk behavior incidence at Wave $2(\beta=0.42, B=16.47, p<$ 0.05). (p. 686) 
International Journal of Child, Youth and Family Studies (2014) 5(4): 493-587

In Harm's Way: A Special Issue on the Impacts and Costs of Witnessing Intimate Partner Violence

However, Begle et al. (2011) also showed that their cross-lag model, while holding for boys, did not do so for girls:

Results of the cross-lag model analyses provided adequate fit to the sample of boys, $\chi 2$ $(92, N=1806)=4773.74, p<0.001, \mathrm{NFI}=0.89, \mathrm{CFI}=0.89, \mathrm{SRMR}=0.23 .[\ldots]$ examination of individual paths revealed that victimization at Wave 1 significantly predicted high risk behavior at Wave $2(\beta=0.03, B=1.55, p<0.05)$ [but] results of the cross-lag model did not provide an adequate fit to the sample of girls, $\chi 2(92, N=1808)=$ 2385.98, $p<0.001, \mathrm{NFI}=0.85, \mathrm{CFI}=0.86, \mathrm{SRMR}=0.12 .($ p. 686)

The four longitudinal studies reviewed above provide several valuable lessons about the development of substance misuse issues: Witnessing violence between one's parents, whether that is termed interparental violence (Fergusson, Boden, \& Horwood, 2008b), family violence (Smith et al., 2010), family conflict (Skeer et al., 2009), or a victimization experience (Begle et al., 2011), makes a direct and lasting contribution to substance misuse. Yet, these studies are not in agreement with respect to gender effects: Smith et al. (2010) suggest that girls are more vulnerable, Begle et al. (2011) and Skeer et al. (2009) suggest that boys are more vulnerable, while Fergusson, Boden, and Horwood (2008b) suggest that both sexes are vulnerable.

Together these studies bring attention to the fact that witnessing IPV has significant impacts on youth substance misuse. As well, methodological problems with cross-study comparability and within study decisions about strategies for data gathering and analysis notwithstanding, the cascading effects of exposure to IPV, whether these cascades are internal (neurohumoral) or external (a series of negative events), are clearly evident in the four longitudinal studies. These findings point to an additional cascade, the cascade of the evergrowing accumulation of evidence of a significant relationship between exposure to IPV and the development of substance misuse issues. We turn next to large representative sample correlational and retrospective studies for further corroboration of the impact of witnessing IPV on youth substance misuse.

Large representative sample correlational and retrospective studies. Additional support for the significant and negative contribution of witnessing IPV to the development of substance misuse issues comes from the large representative sample correlational studies: Douglas et al. (2010); Gavazzi, Lim, Yarcheck, Bostic, and Scheer (2008); Kaufman et al. (2007); Kliewer and Murrelle (2007); Rothman, Edwards, Heeren, and Hingson (2008); Singh, Thornton, and Tonmyr (2011); Strine et al. (2012); Zinzow et al. (2009). These studies, like the longitudinal studies reviewed above, also present a number of methodological issues that create challenges with regard to comparing and synthesizing their results.

The first challenge is that the literature on the factors that contribute to the development of substance misuse largely refers to child maltreatment as a comprehensive construct (see, for example, Hovdestad et al., 2011), without necessarily examining each type of maltreatment separately. As well, the recognition of witnessing IPV as a variable separate from general maltreatment is a relatively recent development. This is confirmed by Gilbert et al. (2009), who 
International Journal of Child, Youth and Family Studies (2014) 5(4): 493-587

In Harm's Way: A Special Issue on the Impacts and Costs of Witnessing Intimate Partner Violence

in their comprehensive search for any systematic reviews of the scholarly literature on child maltreatment and its negative and adult-life persistent impacts published from 2000 to June 2008, found that most of the literature speaks to physical, sexual, and psychological abuse and does not necessarily include mention of witnessing IPV. Still, as these authors also state, "increasingly, witnessing intimate-partner violence is also regarded as a form of child maltreatment" (Gilbert et al., 2009, p. 69). Further, as Gilbert et al. also show, "children who are exposed to one type of maltreatment are often exposed to other types on several occasions or continuously” (p. 71), thus untangling one form of abuse from another in order to measure its individual impact is difficult. It can therefore be assumed that if one category of abuse is found, others are also likely to be present, so even the non-mention of witnessing IPV does not mean that research participants did not in fact witness IPV. Accordingly, Gilbert et al. state, "cross-sectional studies indicate that exposure to multiple forms of abuse and other childhood adversities, including witnessing intimate-partner violence, leads to a cumulative increase in the risk of self-reported alcohol or drug misuse in adulthood” (p. 76). This suggests again that the cascading effect is constantly in play. Indeed, the cross-sectional studies reviewed here, while containing the issues described above, like the longitudinal studies reviewed above, also showed strong links between witnessing IPV and the development of substance misuse problems.

Each of the cross-sectional studies reviewed here made reference to family adversity, although how these were operationalized was far from uniform. Douglas et al. (2010), who retrospectively examined the relationship between adverse childhood events and substance dependence in a sample of 2,510 members of families (average age not reported) receiving services from four United States university health centers, employed constructs that had been developed for the Diagnostic Reliability of the Semi-structured Assessment for Drug Dependence and Alcoholism (SSADDA) drug and alcohol dependence instrument developed by PierucciLagha et al. (2005). The focus of the questions in this instrument is on family stability (multiple caregivers, number of relocations), childhood trauma (exposure to violent crime, sexual abuse, and physical abuse), and relationship with the main caregiver (excellent, very good, good, fair, or poor).

Gavazzi et al. (2008), who examined family-specific factors and their association with poor mental health and substance abuse in adolescents, gathered data about the current experiences of family conflict of their 2,646 court-involved participants in Ohio using the 132-item Global Risk Assessment Device or GRAD (Gavazzi et al., 2003). The GRAD contains 17 items that focus on disrupted family processes including measures of family fights. Kaufman et al. (2007), examining the genetic and environmental predictors of early alcohol use in a group of 219 children (average age 12.5 years) who had been removed from their parents' homes in Connecticut, used child protection case records and multiple informant interviews (caseworkers, parents, and the children themselves) to establish the kinds of abuse the child participants had been subjected to (physical, sexual and emotional abuse, and witnessing domestic violence). Kliewer and Murrelle (2007), who investigated risk and protective factors for adolescent substance use in a three-country Central American sample of 17,215 adolescents aged 12 to 20 years, used a 175-item survey constructed after they conducted a pilot study with 988 adolescents between ages 11 and 19 years in Panama. Their survey included 10 items that 
International Journal of Child, Youth and Family Studies (2014) 5(4): 493-587

In Harm's Way: A Special Issue on the Impacts and Costs of Witnessing Intimate Partner Violence

measured negative family interactions and communication and five items that measured exposure to violence and witnessing serious violence.

Rothman et al. (2008), who looked into the connection between adverse childhood experiences and the early onset of alcohol consumption in a sample of 3,409 current or former drinkers aged 18 to 39 years, used a survey containing questions from the National Epidemiologic Survey on Alcohol and Related Conditions (NESARC), a survey developed by the United States Department of Health and Human Services. With reference to family experiences, this survey contains questions about child abuse and neglect, including psychological abuse, contact sexual abuse, household dysfunction, and witnessing violence against a mother or stepmother (not father or stepfather).

Singh et al. (2011), who investigated the determinants of substance misuse in a random subsample of 4,381 children and adolescents drawn from the Canadian Study of Reported Child Abuse and Neglect-2003 (CIS), a study of 11,552 child maltreatment investigations that included cases from every Canadian province except Québec, used a specifically developed report form that was completed by trained protection workers. The form queried substantial maltreatment and other neglect on a Yes/No basis: Yes - the child experienced any one of substantiated physical abuse, substantiated sexual abuse, substantiated emotional maltreatment, or substantiated exposure to domestic violence; or No - the child did not.

Strine et al. (2012), who examined the connection between adverse childhood experiences, psychological distress as a mediator, and adult alcohol problems in a sample of 7,279 over-18year-old adult members (average age: 54.8 females, 57.1 males) of an American health maintenance organization (HMO), developed and employed the 168-item Family Health History (FHH) questionnaire, that is a composite of selected questions from several previously existing standardized scales, for example, the CTS (Straus, 1979; Straus et al., 1996). The questionnaire included 4 items that queried witnessing violence against participants' mothers or stepmothers (not fathers or stepfathers).

Finally, Zinzow et al. (2009), who examined the relationship between witnessed parental and community-based violence in substance use and delinquency in an American national household probability sample (3,164 parent-child cases) of urban dwelling youth aged 12 to 17 , used a modified version of a previously developed violence assessment module that examined parental violence with five questions about witnessing parents punching, hitting, choking, beating, using objects to beat, or threatening each other with guns, knives, or other weapons. None of the studies, briefly described here, used the same measures, although all covered very similar experiences.

Despite the variations in measures, the range in age of the participants, and the sample size of each of the studies, the collective findings drawn from more than 40,000 participants converged: abuse and lower perceived quality of relationships with their primary caregivers (Douglas et al., 2010), disrupted family processes (Gavazzi et al., 2008), maltreatment, including witnessing family violence (Kaufman et al., 2007), negative family interactions (Kliewer \& Murrelle, 2007), parental discord or divorce (Rothman et al., 2008), substantiated maltreatment 
International Journal of Child, Youth and Family Studies (2014) 5(4): 493-587

In Harm's Way: A Special Issue on the Impacts and Costs of Witnessing Intimate Partner Violence

including substantiated exposure to domestic violence (Singh et al., 2011), and witnessed violence (Zinzow et al., 2009), played a significant role in the development of substance misuse and other issues. Only Strine et al. (2012) did not report a direct connection between family conflict variables (witnessing domestic abuse of mothers and stepmothers) and substance misuse, but the average age of participants was considerably higher than that of the participants in the other studies, and Strine et al.'s emphasis was on psychological distress as a mediator for alcohol problems rather on the direct relationship between the individual variables they queried and substance misuse issues. If we set aside Strine et al.'s 7,279 participants and focus on the findings from the six studies that reported similar age ranges (Kaufman et al., 2007; Kliewer \& Murrelle, 2007; Rothman et al., 2008; Singh et al., 2011; Zinzow et al., 2009) and the data from their 31,034 participants, collectively, these large, representative sample correlational and retrospective studies provide strong evidence that negative family interactions that include witnessing family violence, play a significant role in the development of substance misuse issues.

These correlational studies, taken together with the longitudinal studies described earlier, certainly add to the evidence that implicates witnessing IPV as a source of significant risk for problematic substance misuse issues in adolescence and adulthood. While there may still be room to argue on the basis of the longitudinal studies that the long-term effects of witnessing IPV may only pertain to females (Smith et al., 2010), or that females and males are both affected but males ultimately experience an even stronger effect (Begle et al., 2011; Skeer et al., 2009), the additive weight of the research provides a strong case for taking seriously the impact of witnessing IPV on the development of substance misuse issues, especially because substance misuse is also strongly implicated in delinquency and crime.

\section{Delinquency, Crime, and Victimization}

Much of the current research examining the link between exposure to domestic violence and violent or aggressive behaviour has examined compounding factors, such as that of exposure to domestic violence and adolescent maltreatment (Mejia, Kliewer, \& Williams, 2006; Park, Smith, \& Ireland, 2012; Sousa et al., 2011). Both targeted child abuse and IPV contribute substantively to an array of problematic outcomes. This overview summarizes the current research on the effects on children and youth of the adversity package (Jirapramukpitak et al., 2011), that is, the "family burden" (Kassis et al., 2013) of the exposure to family violence and delinquency and crime. However, although both witnessing IPV and experiencing violence are associated with the highest level of negative adjustment problems, exposure to IPV only is also associated with delinquency and substance use (Ellonen, Piispa, Peltonen, \& Oranen, 2013).

Although we chose to focus our attention on research conducted from 2006 onwards, for our consideration of crime, delinquency, and victimization, we also believed it important to include four earlier reviews that covered additional material from 1994 to 2003 and provided the basis for the more recent reviews upon which we concentrated. The first of these four reviews, Schwartz, Rendon, and Hsieh (1994), still focused on child maltreatment rather than children's exposure to aggression and violence between their parents or caregivers, but nonetheless drew our attention to some key issues in the research that has been generated about family violence and child abuse and their effects: Although a link has been established between family violence, 
International Journal of Child, Youth and Family Studies (2014) 5(4): 493-587 In Harm's Way: A Special Issue on the Impacts and Costs of Witnessing Intimate Partner Violence

particularly child maltreatment and neglect, and delinquency, the wide range of methodologies for examining these links, the notable variation in definitions for what constitutes violence, maltreatment, and delinquency, and the possible selection biases in sample populations, create challenges for those who attempt to systematically analyze this literature. Schwartz et al. therefore suggest that where reviews of this literature are concerned, one should tread carefully and select for inclusion only those studies that have rigorously dealt with the issues outlined above. That is, studies that: (a) employed comparable methodologies that could be independently replicated; (b) were based on prospective rather than retrospective data; (c) utilized comparable standardized tools and definitions; and (d) accounted for sampling biases such as drawing only from women's shelters and transition house populations and from present and former children in care or from incarcerated youth and adults.

Buehler et al. (1997), in their meta-analysis of interparental conflict and youth problem behaviours, did just as Schwartz et al. (1994) suggested and from a body of literature that spanned 15 years, selected 68 studies that met the required criteria for definitional agreement and sampling and for data that allowed the calculation of effect size. After conducting their rigorous meta-analysis, Buehler et al. reported an average effect size of .32 and noted "a consistent and important association between inter-parental conflict and youth problem behaviors” (p. 243). Following Buehler et al. (1997), Kitzmann et al. (2003) also conducted a meta-analysis of the psychosocial outcomes of children exposed to family violence. In their analysis, Kitzmann et al. acknowledged the earlier work of Buehler and others but noted that this work included in a wide spectrum of forms of marital discord and conflict strategies, not only exposure to physical aggression between parents. Although Buehler et al. made an important contribution to our overall understanding of the effect of interparental conflict, their analysis does not inform us specifically about the effects of witnessing interparental violence. To that end, Kitzmann et al. (2003) examined 118 studies (84 journal articles, 5 book chapters, and 29 theses or dissertations) published between 1978 and 2000. The selected studies allowed outcome comparisons for: (a) child witnesses of interparental violence and non-witnesses; (b) child witnesses of interparental violence and child witnesses (only) of interparental verbal aggression; (c) child witnesses of interparental violence and children who had been physically abused; and (d) child witnesses of interparental violence and physically abused children, along with a systematic comparison of the reported outcomes of correlational studies of exposure to the four conditions described above. All 118 selected studies yielded a significant association between exposure to interparental aggression and/or violence and to physical abuse and poor child outcomes. Witnessing interparental violence creates a notable risk, one that is at least as problematic as direct abuse at the hands of one's parents.

Wolfe et al. (2003), who conducted their meta-analysis almost concurrently with Kitzmann et al. (2003), and reference Kitzmann and colleagues, selected only 41 studies for their analysis. Still, Wolfe et al.'s analysis, while premised on even more conservative methodological parameters than Kitzmann et al.'s, shows considerable overlap with that of Kitzmann et al. (33 of the 41 studies analyzed by Wolfe et al. were the same as those included by Kitzmann et al.). Not surprisingly, Wolfe et al. and Kitzmann et al. came to similar conclusions. Wolfe et al. therefore state that the "answer to whether or not children exposed to violence experience more difficulties than their peers emerged as an unequivocal yes” (p. 183). 
International Journal of Child, Youth and Family Studies (2014) 5(4): 493-587

In Harm's Way: A Special Issue on the Impacts and Costs of Witnessing Intimate Partner Violence

By 2003, based on three meta-analyses of 194 studies, some of which were analyzed twice, a convergence of findings had emerged: Exposure to violence in the family has serious consequences for a significant number of children, a number that should not be ignored. Still, up to this point, the analyses focused on a range of poor externalized and internalized psychosocial outcomes rather than examining the effects of exposure to violence on each of these outcomes separately. This problem persists even in more recent work and should perhaps be acknowledged not as a limitation of that work but as likely an accurate reflection of the reality that poor child and youth outcomes cannot be neatly sorted into discrete non-overlapping categories. This overlap, and with it the inability to neatly pinpoint which outcomes are tied to which outcomes, necessitates an understanding of the impacts of domestic violence that is dynamic even while striving to sort out differential impacts and outcomes.

A dynamic understanding of the impact of exposure to domestic violence on the health and development of children is summarized in Holt et al.'s (2008) review of 143 peer-reviewed articles selected from over 1,000 studies conducted in the 11-year period between 1995 and 2006. This review, which also included research that was previously analyzed (for example, Kitzmann et al., 2003; Wolfe et al., 2003), focused specifically on those studies that examined children and young people's outcomes through reports, surveys, and other measures that directly involved these children and youth rather than eliciting the views of their parents, shelter workers, and other professionals as a way to gauge these outcomes. This focus on child and youth experience rather than on others' (mediated) perspectives of that experience is what lends particular significance to Holt et al.'s review. As Holt et al. point out, although we have more than three decades of research on the scope and consequences of children's exposure to domestic violence it is only within the last decade or so, that we have begun to acknowledge that children are indeed "dynamic in their efforts to make sense of their experiences while navigating their way around the complexity and terror intrinsic to domestic violence” (p. 798).

Holt et al. underline the fact that children are not just passive recipients of experience recipients who merely replicate and reproduce what they see, hear, taste, touch, and feel; they also interpret their experiences and use their interpretations to draw conclusions about self, others, and the world. Further, as Holt et al. show, as children grow and develop, they shift and change their understanding of and their responses to exposure to domestic violence. Thus the impacts of domestic violence are developmentally linked and negative child outcomes are, as Wolfe et al. (2003) noted, processes rather than once and for all absolutes.

\section{Developmental Pathways}

The impact of frequent exposure to IPV may have long-term negative outcomes on a child's aggressive and externalizing behaviour. For instance, one American study found no significant differences in aggressive behavioural problems among children exposed to frequent IPV between birth and 3 years of age and their non-exposed peers (Holmes, 2013). However, over time, the children who were more frequently exposed between birth and 3 years of age were found to exhibit more aggressive behaviour 5 years later (Holmes, 2013). Thus, where the impacts of domestic violence on youth delinquency and crime and later adult crime are 
International Journal of Child, Youth and Family Studies (2014) 5(4): 493-587

In Harm's Way: A Special Issue on the Impacts and Costs of Witnessing Intimate Partner Violence

concerned, the pathway is also developmental. For example, the first manifestations of later behavioural and criminal problems may appear as: problems with attachment for infants and toddlers (McIntosh, 2002); behavioural problems like temper tantrums and aggression in preschoolers (Cunningham \& Baker, 2004; Huth-Bocks, Levendosky, \& Semel, 2001); antisocial rationales (Cunningham \& Baker, 2004); difficulties with aggression and adhering to rules, poor social skills, engagement in bullying others, being bullied during the school-age years (Bauer et al., 2006; Cunningham \& Baker, 2004; Knous-Westfall, Ehrensaft, MacDonell, \& Cohen, 2012; Lundy \& Grossman, 2005; Voisin \& Hong, 2012); and then delinquency and crime in adolescence and adulthood (Leschied, Chiodo, Nowicki, \& Rodger, 2008).

Leschied et al. (2008) took up the developmental understanding of criminal behaviour in their meta-analysis of 38 prospective and longitudinal studies that examined the impact of negative early childhood experiences and detrimental family factors on adult offending in a total of 66,647 participants (68\% from continental Western Europe; 24.7\% from the United States; 6.3\% from the United Kingdom, Australia, and New Zealand) who were followed from a mean age of 10.5 until they reached a mean age of 24.5. Leschied et al. also emphasized dynamic over static characteristics of experience and calculated effect sizes for the factors that the 38 studies reported. They found that overall, across all age groups, externalizing issues (e.g., lack of selfcontrol, antisocial behaviour, hyperactivity, and conduct disorder) were satisfactory predictors of adult criminality with the predictive strength becoming stronger as the behavioural issue persisted over time (effect size was .20 in early childhood, .31 in mid-childhood, and a robust .52 in adolescence). As well, the most potent family factors that were predictive of adult criminality across all studies were: parent management that is coercive, inconsistent, lacking in supervision, and a child or youth becoming involved with the child welfare system.

\section{Parenting and Delinquency}

In their meta-analysis of 161 published and unpublished manuscripts about the association of parenting and delinquency, Hoeve et al. (2009) point out that parenting should not be examined in isolation from an examination of other familial factors, but interestingly confine their analysis to a range of parenting behaviours without making reference to either contextual or interfamilial factors. Still, they provide convincing evidence for a significant relationship between harsh, hostile, rejecting, controlling, and neglectful parenting with delinquency, evidence that again raises the spectre of an overall negative family climate. Lescheid et al. (2008) did include family dynamics in their meta-analysis and found adverse family environments that include family violence implicated in the question of dynamic risk and the prediction of criminality. They state:

[T]he nature of parent management viewed as inconsistent, harsh, or punitive, along with a child's experience with violence in the home, both as a witness and as a victim proved to be particularly strong family-based predictors [...] it is the combination of factors as well as the intensity of a specific risk factor that leads to the overall potency of prediction [...] child-specific risk - including a variety of behavioral disorders in combination with [italics in the original] being the recipient of harsh or inconsistent parenting and exposure 
International Journal of Child, Youth and Family Studies (2014) 5(4): 493-587

In Harm's Way: A Special Issue on the Impacts and Costs of Witnessing Intimate Partner Violence

and victimization of violence within the home that can co-occur or follow in a sequence of risk - can incrementally increase the accuracy of [risk] prediction. (p. 458)

Criminality, like many social problems, cannot be traced to a single cause or contributing factor but violence in the family - whether in the form of targeted abuse, hostile, harsh and punitive parenting, exposure to IPV, or a combination of these - creates serious risk for criminality and other negative outcomes. While the precursors to criminality may be experienced in the private domain of the family home, the impacts that emanate from that private experience are felt far beyond the family domain and very quickly become a broadly shared concern.

Violence that is enacted upon family members by family members is, of course, a chargeable offence; it is a crime. The extent of this crime in Canada has been under careful scrutiny for some time and has been the focus of the Family Violence Initiative or FVI (Public Health Agency of Canada, 2013) since 1998. The FVI is a comprehensive Government of Canada program led by the Public Health Agency of Canada and involving 15 departments, agencies, and Crown corporations working in concert to reduce the occurrence of family violence. Each year, the FVI, in conjunction with Statistics Canada, releases an annual report that tracks the nature and extent of family violence in this country. It is of great importance to our understanding of the impact of family violence on crime, and the intergenerational transmission of this criminal behaviour, that we take a close look at the role of family violence in the overall distribution of crime in Canada and that we note the developments in our understanding that have occurred in recent years. The following excerpt from the 2010 Family Violence in Canada Statistical Profile illustrates this point:

Recently, there has been some consideration within the research community both nationally and internationally and by federal, provincial and territorial governments towards including all types of intimate partner relationships, including dating partners, in a definition of family violence [...] Violence against dating partners has been argued to fall within the definition of family violence, due to its many similarities with spousal violence [...because] research has found that the characteristics of police-reported dating violence generally mirror those of spousal violence [...and because] research has [also] found that individuals' experiences in early dating relationships can have an impact on future patterns of violence for both victims and abusers. (Varcoe et al., 2011, p. 9)

The inclusion of dating violence in the definition of IPV highlights two important findings in the Canadian context:

1. The victimization rate for police-reported IPV is 341/100,000 for people aged 15 years and older, a rate that is almost 2.5 times higher than police recorded rates for family violence against children, parents, or other family members where the reported rate is 141/100,000; and

2 . Even when compared to the police-reported rate for stranger violence $(287 / 100,000)$, the greatest risk for victimization is to be found in intimate partner relationships (Sinha, 2013).

These rates not only speak to victimization, they also provide a record of chargeable offences, that is, criminal activity. It is especially in the perpetration of IPV that the 
International Journal of Child, Youth and Family Studies (2014) 5(4): 493-587

In Harm's Way: A Special Issue on the Impacts and Costs of Witnessing Intimate Partner Violence

intergenerational transmission of crime is most apparent. As noted by Schwartz, Hage, Bush, and Burns (2006), in their narrative review of the 143 studies on witnessing and/or experiencing violence in the family and poor parenting practices generated since 1984, "witnessing and/or experiencing violence in the family of origin has been the most researched and accepted explanation for the intergenerational transmission process of intimate violence” (p. 206). They, too, suggest that there is no single mechanism or element at the core of the intergenerational transmission of IPV and suggest that rather than attempting to isolate single factors or characteristics in the search for a causal model, we consider instead what they describe as "multiple family-of-origin pathways to future intimate violence” (p. 207).

This model embraces the overlap between witnessing IPV and child abuse - also identified by Bedi and Goddard (2007) - and recognizes, as noted earlier in this review, that the impacts of domestic violence are dynamic rather than chain-like. As Schwartz et al. (2006) show, "detrimental family-of-origin factors not only model violent, abusive, and coercive behaviors but also have negative effects on intrapersonal (e.g., self-worth) and interpersonal (e.g., communication skills) development [and] may also damage the attachment between parent and child" (p. 216) and thereby model maladaptive relational templates. These maladaptive templates serve to continue the intergenerational transmission of intimate partner violence by ill equipping the next generation for positive attachment and by modelling violent crime toward those with whom one has one's closest connections.

In a study comparing a representative sample of 1,152 school-aged children to 148 juvenile offenders between the ages of 11 and 19 in Colombia, Mejia et al. (2006) examined the associations between direct and indirect mechanisms of family violence and adolescent maltreatment with aggression and impaired prosocial behaviour. These researchers found that exposure to domestic violence was both directly $(.06, p<.001)$ and indirectly $(.09, p<.001)$ associated with violent behaviour. They also found that children who were exposed to both family violence and childhood maltreatment "reported greater levels of violent behaviors, such as carrying weapons, knives, or responding by physically fighting, and limited capacity to respond proactively towards others” (Mejia et al., 2006, p. 265). Similarly, Park et al. (2012) used longitudinal data collected from youths 14 years old to adulthood, from the RYDS, to explore the co-occurrence of domestic violence exposure and child maltreatment and found that, although maltreatment has a stronger overall impact on negative behavioural outcomes such as antisocial or violent behaviour in both adolescence and adulthood, exposure to severe IPV does have an independent impact on committing violent crime.

Being exposed to domestic violence in youth may also have long-term negative consequences that result in other violent or criminal behaviour. Witnessing IPV in childhood may increase the risk of perpetrating aggressive behaviour or IPV during adolescence and adulthood (Adams, 2009; Caldeira \& Woodin, 2012; Fergusson et al., 2008b; Lohman, Neppl, Senia, \& Schofield, 2013; Mandal \& Hindin, 2013; Moretti, Obsuth, Odgers, \& Reebye, 2006; Murrell, Christoff, \& Henning, 2007; Roberts, Gilman, Fitzmaurice, Decker, \& Koenen, 2010; Temple, Shorey, Tortolero, Wolfe, \& Stuart, 2013) and may be associated with cognitive biases which condone abusive behaviour (Neighbors et al., 2013). 
International Journal of Child, Youth and Family Studies (2014) 5(4): 493-587 In Harm's Way: A Special Issue on the Impacts and Costs of Witnessing Intimate Partner Violence

Fergusson et al. (2008b) conducted a study that examined the developmental antecedents of IPV victimization and perpetration in a New Zealand birth cohort $(n=828)$. The researchers found that individuals exposed to multiple psychosocial adversities in childhood, including family adversities in adolescence such as child abuse or family violence, and who showed longterm adjustment difficulties, were at a higher risk of domestic violence perpetration. Similarly, Moretti et al.’s (2006) study provides evidence that exposure to interparental violence increases an adolescent's risk of perpetrating aggressive acts against mothers, fathers, friends, and romantic partners. In their sample of 63 girls and 49 boys (ages 13 to 18), Moretti et al. found that females who witnessed their mothers' aggressive behaviour toward their partners were significantly more aggressive toward their peers and friends, whereas male youths who were exposed to their fathers' aggression were significantly more aggressive toward their friends. Both male and female adolescents who observed their mothers' aggression toward their partners also reported significantly higher levels of aggression toward their romantic partners. Murrell et al. (2007) examined a sample of 1,099 adult males arrested for battery and also found that as exposure to domestic violence as a child increased, so did the frequency of domestic violence offences perpetrated.

Social learning theory may play a role in the perpetration of domestic violence during adulthood by individuals who were exposed to domestic violence in childhood. Adams (2009) examined data collected from in-depth interviews with 51 men who killed or attempted to kill their partners. Adams' (2009) research supports the social learning theory of domestic violence that it is "first learned by parenting modeling and then socially reinforced through peer and cultural supports of male dominance within male-female relationships” (p. 228). However, Gover, Jennings, Tomsich, Park, and Rennison's (2011) study examining social learning and self-control theories in a sample of South Korean and American college students found that observing domestic violence in childhood "was not a predictor of psychological violence in the college student samples", which may indicate that "modeling does not appear to cross types of violence (i.e., physical and psychological)” (p. 311).

Exposure to either domestic violence or child abuse has also been found to affect a child's attachment to their parents and antisocial behaviour (Sousa et al., 2011). Youth who have a stronger attachment to their parents have lower risks of antisocial behaviour during adolescence (Sousa et al., 2011). The connection between in-home exposure to IPV and subsequent aggressive and antisocial behaviour is also well supported by recent longitudinal research conducted by Ireland and Smith (2009) who followed 1,000 urban American youth from age 14 to adulthood using prospective data gathered in the RYDS (Browning, 1999; Thornberry, Krohn, Lizotte, Smith, \& Porter, 1998), a study that examined the impact of family process factors on delinquency and youth development. Ireland and Smith's findings are compelling. Using bivariate and multivariate logistical regression, they showed that: (a) the risk of violent crime in adolescence is 1.7 times greater if the adolescent resides in a partner-violent family; (b) caregiver severe partner violence is significantly related to self-reported violent crime; (c) being raised in a partner-violent family significantly increases the risk of both antisocial behaviour and relationship violence in early adulthood; and (d) none of the differences between male and female coefficients achieved statistical significance $(p<.05)$. 
International Journal of Child, Youth and Family Studies (2014) 5(4): 493-587

In Harm's Way: A Special Issue on the Impacts and Costs of Witnessing Intimate Partner Violence

Even the non-violent aspects of interparental conflict are implicated in the transmission of intimate partner violence, specifically in dating violence in adolescence (Tschann et al., 2009). In their longitudinal study of 150 Mexican-American and European-American male and female adolescents, Tshann et al. used multiple regression and path analyses and found that both nonviolent parental conflict and interparental violence were predictive of mutual verbal aggression, dating violence victimization, especially for girls, and dating violence perpetration for both sexes, especially for boys. Both sexes were affected by exposure to parental conflict and violence and both sexes were at significant risk for becoming victims and/or perpetrators of romantic partner violence, and therefore, at heightened risk for involvement in criminal behaviour.

If exposure to interparental violence is tantamount to child abuse (Bedi \& Goddard, 2007), and such exposure is at the same time significantly predictive of future involvement in delinquency, crime, and the use of violence against romantic partners, then surely a concerted effort aimed at preventing intimate partner and romantic partner violence would make a strong contribution to crime prevention. As Sinha (2013) documents:

Intimate partner violence, including both spousal and dating violence, accounts for one in every four violent crimes reported to police. In 2011, there were approximately 97,500 victims of intimate partner violence, representing a rate of 341 victims per 100,000 population [...] Violence by a dating partner was more prevalent than violence committed by any other type of perpetrator, including one of the most common - friends or acquaintances. In particular, the rate of violence by a dating partner was $10 \%$ higher than the rate of violence committed by friends or acquaintances, $42 \%$ higher than stranger violence and nearly three times higher than non-spousal family violence. (p. 38)

It would seem then that for many people, the perpetration of crime and the experience of victimization are most closely connected to those with whom they have their closest relationships.

\section{Interpersonal Victimization}

In addition to the perpetration of crime, youth exposed to domestic violence are at an increased risk for criminal and violent victimization compared to non-exposed youth (Mitchell \& Finkelhor, 2001; Schewe, Riger, Howard, Staggs, \& Mason, 2006), which may compound the mental health impacts of early IPV exposure. Mitchell and Finkelhor (2001) examined data from the 1996, 1997, and 1998 American National Crime Victimization Surveys and compared survey participants of households with at least one youth who was exposed to: (a) at least one incident of domestic violence against an adult ( $n=352$ households, 627 youth); (b) at least one incident of violence against an adult by a nondomestic person ( $n=1,993$ households, 3,534 youth); or (c) no crime or violence against an adult ( $n=2,333$ households, 4,106 youth). The study authors found that living in a household with domestic violence increased both boys' and girls' risk of victimization by $115 \%$ and $229 \%$, respectively, as opposed to youth in households with no domestic violence (100\% and 119\%). Although some of these youths were victimized by the same perpetrator that committed domestic violence against an adult in their household, "most of the victimizations of youth from domestic violence households were at the hands of someone 
International Journal of Child, Youth and Family Studies (2014) 5(4): 493-587

In Harm's Way: A Special Issue on the Impacts and Costs of Witnessing Intimate Partner Violence

outside of the family, suggesting that the impact of living in a violent household extends beyond interfamilial abuse” (Mitchell \& Finkelhor, 2001, p. 958).

In another study examining welfare recipients in Illinois, Schewe et al. (2006) used data collected from a third wave of interviews with 814 women receiving welfare services and found

that being exposed to domestic violence as a youth was a significant risk factor for sexual assault $(p<.01)$ and domestic violence victimization $(p<.001)$ in adulthood. The lifelong impacts of childhood exposure to IPV, however, do not end here. Even if this exposure does not lead to internalizing and or externalizing mental health and behavioural issues, substance misuse, delinquency and crime or victimization, there are additional risks pertaining to academic and employment outcomes, as discussed in the following section.

\section{Academic and Employment Outcomes}

The link between educational attainment and economic success is well demonstrated (Alexander, 2011; Currie \& Widom, 2010; Macmillan \& Hagan, 2004; Tanaka et al., 2011; Zielinski, 2009). According to Zielinski (2009), "better performance in school and higher educational attainment leads to higher income, better odds for employment, and more stable employment over time” (p. 675). Therefore, it is vital to consider educational deficits when considering the ongoing impacts of IPV exposure and employment outcomes. Zielinski further noted that, "maltreatment has consistently been found to serve as a significant risk factor for impaired cognitive and academic outcomes" (p. 675). She also cited several studies that demonstrated the negative effects of early victimization on educational attainment and highlighted the fact that maltreatment in childhood was associated with a higher risk of not completing high school.

Margolin and Gordis (2000) created a substantial list of additional functional deficits with lasting ramifications for children exposed to trauma. These effects include delayed cognitive development, poor academic performance, inability to react to stress adequately, dysregulation of the stress regulating system (i.e., the hypothalamic-pituitary-adrenal axis), abnormal hypothalamic pituitary growth hormone axis control, and limbic system dysfunction. Impairments in physical functioning, including body pain, general health, and vitality are also correlated with IPV (Dauvergne \& Johnson, 2001; Tanaka et al., 2011).

Detrimental experiences that influence child development and pivotal transitions shape and alter the life course of individuals (Macmillan \& Hagan, 2004). Self-report data on witnessing regular and consistent parental violence in adolescence is negatively associated with income and wealth, that is, net worth (Covey, Menard, \& Franzese, 2013). This section of the literature review was written with the intention of explicating the relationship between exposure to IPV and employment outcomes. At this stage of the analysis, existing research indicates that there are a myriad of factors associated with IPV that put individuals at risk for problematic employment trajectories and future achievements. Although reviewed in summary, the psychopathological problems, psychosocial difficulties, and functional deficits convey general barriers to successful employment. However, the lack of research in this area reveals that relatively little is known about the ongoing consequences of IPV and future socioeconomic 
International Journal of Child, Youth and Family Studies (2014) 5(4): 493-587

In Harm's Way: A Special Issue on the Impacts and Costs of Witnessing Intimate Partner Violence

outcomes. "This gap in our knowledge is surprising considering recent speculation that exposure to violence undermines socioeconomic attainments” (Macmillan \& Hagan, 2004, p. 128). The current findings reinforce the need for new and continued investigation of the influence of IPV on employment variables. At this point, the impacts of IPV appear to lead to possible, and perhaps significant, socioeconomic disadvantage in the long run. With specific and applicable knowledge, subsequent intervention and prevention models may be able to moderate this particular effect.

\section{Academic Outcomes}

Exposure to domestic violence has been considered a form of child maltreatment, which is known to impede children's academic performance and success (Coohey, Renner, Hua, Zhang, \& Whitney, 2011). Yet, not all children who have been exposed to domestic violence have difficulties in school (Coohey et al., 2011). Because young children are disproportionately exposed to domestic violence, and are more limited in their ability to cope with the impacts of domestic violence, the effects of IPV exposure on early learning may be especially concerning (Baker \& Cunningham, 2009).

Intellectual ability. Domestic violence impacts children's cognitive development by increasing stress levels and by impacting mothers' own mental health, which can in turn affect their relationships with their children (Busch \& Lieberman, 2010). There is some evidence that multiple forms of adversity in childhood may be linked to IQ, with research indicating that children exposed to domestic violence have lower IQ scores than non-exposed children (Busch \& Lieberman, 2010; Byrne \& Taylor, 2007). According to Busch and Lieberman, this relationship may be moderated by mothers' attachment security, as their study found mothers' attachment to be associated with IQ scores, particularly verbal IQ scores, of preschool-aged children who had witnessed at least one incident of IPV in the previous year. A pilot study examining the relationship between children's exposure to trauma (including direct physical/sexual abuse and witnessing domestic violence) and deontic reasoning concluded that dissociation, rather than trauma exposure, predicted errors in this type of reasoning among school-aged children (DePrince, Chu, \& Combs, 2008).

One longitudinal study (Coohey et al., 2011) used the Violence Exposure Scale to measure maltreated children's $(n=702)$ exposure to domestic violence in the form of witnessing IPV. Only $12.8 \%$ of the children in the sample reported domestic violence exposure and the study found that, although domestic violence exposure was related to reading scores, it was not related to children's overall academic achievement (Coohey et al., 2011). Exposure in this study was a measure of children's reports of witnessing domestic violence, rather than hearing or knowing about domestic violence. Another longitudinal study found that 12-year-old children who had witnessed family violence were 1.45 times more likely than non-witnesses to have low reading levels (Thompson \& Whimper, 2010).

Cognitive development and intellectual ability, particularly verbal skills, are important for academic success (Graham-Bermann, Howell, Miller, Kwek, \& Lilly, 2010). IPV exposure has been found to be associated with speech and language difficulties, with IPV-exposed 
International Journal of Child, Youth and Family Studies (2014) 5(4): 493-587

In Harm's Way: A Special Issue on the Impacts and Costs of Witnessing Intimate Partner Violence

children 7.5 times more likely to have been referred to a school-based speech pathologist than their non-exposed peers (Kernic et al., 2002). Another study found that mother-reported exposure to IPV for infants at 30 months old significantly predicted lower memory functioning for the children when they were 60 months old and entering school: When controlling for confounding variables, IPV exposure was able to predict lower short-term $(\beta=-.46, p<.01)$, working $(\beta=-.17, p<.05)$, and deliberate $(\beta=-.15, p<.05)$ memory (Gustafsson et al., 2013$)$. Other research has found that children exposed to domestic violence scored significantly lower on standardized tests of verbal ability than a national sample of same-aged children, and that this relationship was mediated by maternal education level (Graham-Bermann et al., 2010). A longitudinal study found that secure maternal attachment was associated with higher IQ scores in children who had been exposed to domestic violence, thus suggesting that parents play an important role in moderating the impact of domestic violence on their children (Busch \& Lieberman, 2010). Ybarra, Wilkens, and Lieberman’s (2007) research also found that among 2to 5-year-old children, those who witnessed domestic violence had lower verbal scores, poorer cognitive functioning, and higher internalizing behaviours than their non-exposed peers.

Academic performance. The overall association between IPV exposure and academic outcomes has been described as ambiguous (Knutson, Lawrence, Taber, Bank, \& DeGarmo, 2009), though research has found exposure to physical marital aggression and violence to be associated with poor academic progress and performance (Haeseler, 2006; Jayasinghe et al., 2009; Margolin, Vickerman, Oliver, \& Gordis, 2010; Peek-Asa et al., 2007). A study of Chinese adolescents in Hong Kong found witnessing domestic violence to be associated with cognitive functioning $(-0.13, p<.05)$, as measured by self and school reports of academic performance (Ho \& Cheung, 2010). In this sample of 422 students (65\% male, 35\% female) aged 11 to 18, girls reported more exposure to (i.e., witnessing) domestic violence than boys. In a crosssectional study of 828 Sri Lankan children, aged 14 to 17, IPV exposure was found to be negatively associated with both school performance and attendance. Children who were exposed to current physical abuse of their mothers were found to be 2.8 times more likely to obtain an average grade of $<40 \%$ (i.e., a failing grade) and 3.8 times more likely to have school attendance $<80 \%$, compared to their non-exposed peers (Jayasinghe et al., 2009).

A prospective, longitudinal study of 103 families found that children aged 9 and 10 who were exposed to physical marital aggression in the previous year were more likely to experience academic failure, as measured by failing grades, suspension, and/or expulsion (Margolin et al., 2010). Over half (59.2\%) of the children in this sample reported being exposed to physical marital aggression at least once in the previous year, providing further evidence that a large number of children are exposed to IPV each year, and that this exposure has significant implications for their educational attainment and academic success.

Academic performance may be affected by exposure to domestic violence in a number of ways, both as a result of the negative effects of exposure on children and the impact of domestic violence on victims' ability to support their children's academic success. For instance, children may have difficulty focusing or concentrating because they are preoccupied with problems at home or are getting insufficient sleep (Buckley et al., 2007; Peek-Asa et al., 2007). The coping strategies of young children, while perhaps adaptive in the environment in which domestic 
International Journal of Child, Youth and Family Studies (2014) 5(4): 493-587

In Harm's Way: A Special Issue on the Impacts and Costs of Witnessing Intimate Partner Violence

violence is occurring, may not be adaptive in school settings and may interfere with their academic performance (Baker \& Cunningham, 2009); that is, children who tune out noise to cope at home may similarly tune out teachers in the classroom (Baker \& Cunningham, 2009). Some children and youth have difficulty studying or completing homework assignments due to ongoing conflict at home, which can in turn lead to conflict with teachers and detachment from school (Buckley et al., 2007; Peek-Asa et al., 2007). IPV exposure can also have an impact on children's emotional expression, which may interfere with their academic performance, as well as relationships with teachers and peers (Byrne \& Taylor, 2007).

Research suggests that children who are exposed to domestic violence may not reach developmental or academic milestones as quickly as their peers (Baker \& Cunningham, 2009). A rural population-based cohort study involving school-aged children found that those exposed to severe physical domestic violence scored 12.2 percentile points lower, on average, than their non-exposed peers, on standardized tests (Peek-Asa et al., 2007). The study found that standardized test score reductions were greater for girls than for boys, and for younger (i.e., under age 12) than older children. Additionally, the effects were stronger for language and math than for reading. The authors note, however, that they did not examine direct child abuse and that, given the strong correlation between abuse and IPV exposure, it is plausible that other confounding variables, such as child abuse, were responsible for test score reductions. However, another study of children exposed to maternal IPV found that exposure was not related to lower grades, special education, or being held back in school - all measures of poor academic performance. Yet, if children were also themselves abused, their GPAs were lower, on average, than those of their non-exposed peers, and they were at greater risk of being held back a grade in school (Kernic et al., 2002).

Absenteeism and suspension. Domestic violence may also be one reason that children move or change schools, which has the potential to disrupt their learning and affect their academic success (Baker \& Cunningham, 2009). In some cases, the impact of domestic violence on children and youth may result in absenteeism or dropping out, suspension or expulsion from school, or being denied access to education (Byrne \& Taylor, 2007). However, few teachers in Byrne and Taylor's (2007) study reported expulsion for children who displayed aggressive behaviour at school and were exposed to domestic violence at home. A study of Sri Lankan children found that those exposed to physical violence against their mothers were more likely to have both poor school attendance and performance (Jayasinghe et al., 2009). Another study of children exposed to domestic violence found them to be 1.6 times as likely to be absent from school, and 1.8 times as likely to be suspended from school, than their non-exposed peers (Kernic et al., 2002). Importantly, the likelihood that exposed children would be absent from school rose to 2.2 times the comparison group if those children were also themselves the victims of child abuse. The study did not find maternal IPV exposure to be associated with academic expulsion (Kernic et al., 2002). One longitudinal study found that the relationship between domestic violence exposure and dropping out of high school was moderated by peer communication and support (Tajima, Herrenkohl, Moylan, \& Derr, 2011).

Spillover effect on peers. The impact of IPV exposure on school-aged children is not limited to children who are directly affected, but may also have residual effects on their peers. In 
International Journal of Child, Youth and Family Studies (2014) 5(4): 493-587

In Harm's Way: A Special Issue on the Impacts and Costs of Witnessing Intimate Partner Violence

a study that matched school records with domestic violence cases, identified through civil court records involving a request for protection, Carrell and Hoekstra (2010) estimated that adding "one more troubled peer in a classroom of 20 students reduces student [reading and math] test scores by 0.69 percentile points and increases the number of disciplinary infractions committed by 17 percent” (p. 226). The authors note that these effects were amplified for higher-income children whose lower-income peers have been exposed to domestic violence, and for boys whose male peers have been exposed to domestic violence. The "spillover" of domestic violence exposure for one child thus has impacts for other students in the classroom. It is unclear from this study whether negative academic outcomes stem from the disruptive behaviours of IPV-exposed students in the classroom that distract non-exposed peers or are observed because IPV-exposed students are slower learners, thereby slowing down the learning of other students. This study highlights the important implications of domestic violence exposure not only for children who live in households where such violence occurs, but also for their peers who may be indirectly exposed to the impacts of IPV at school.

While difficulties at home may interfere with academic success, not all children who are exposed to domestic violence at home will exhibit behavioural difficulties in school. Rather, Byrne and Taylor (2007) suggest "schools may be seen as a protective or resilience factor against the effects of domestic violence on children” (p. 187). Some exposed children adjust well to new school settings and make a concerted effort to hide the impact of domestic violence exposure, making it more difficult for school staff to identify those who are experiencing adversity at home (Buckley et al., 2007; Turpel-Lafond, 2012). Children may perceive school as a "safe place" where they are not at risk of exposure and where they may find some support to deal with the impact of domestic violence exposure (Buckley et al., 2007; Byrne \& Taylor, 2007).

Young children may have limited coping skills when facing exposure to domestic violence and, because educators are in contact with students on a regular basis, they play an important role in identifying signs of domestic violence exposure, supporting children as they attempt to cope with the impact of domestic violence, serving as positive role models for children, and enhancing children’s safety (Baker \& Cunningham, 2009; Haeseler, 2006). Specifically, teachers may provide positive and supportive relationships that can assist children in coping with domestic violence in the home (Byrne \& Taylor, 2007). However, one study involving a focus group with 11 abused mothers, found that school staff sometimes lacked understanding about domestic violence, and were unable or unwilling to assist children who were exposed to violence in the home (Buckley et al., 2007).

Interviews with youth who were themselves exposed to domestic violence similarly found that programming for school-aged children and teachers was thought to be beneficial so that teachers were more understanding, supportive, and inclusive of children who were exposed to domestic violence (Buckley et al., 2007). A recent report from British Columbia’s Representative for Children and Youth recommended domestic violence training for school personnel throughout the province, and stressed the importance of age-appropriate education for school-aged children concerning issues of domestic violence (Turpel-Lafond, 2012). 
International Journal of Child, Youth and Family Studies (2014) 5(4): 493-587

In Harm's Way: A Special Issue on the Impacts and Costs of Witnessing Intimate Partner Violence

\section{Employment Outcomes}

Given the detrimental impacts of exposure to IPV in childhood and adolescence on the life course path, one could hypothesize that childhood adversity may substantially influence the future employment outcomes and earning potential of individuals. Entering the labour force is a key developmental stage of adolescence. While employment enhances psychosocial functioning and provides a myriad of benefits, failure to execute this transition successfully can lead to serious problems in adulthood (Tanaka et al., 2011; Zielinski, 2009). Unfortunately, the association between exposure to IPV in childhood and adolescence, and employment outcomes, has not been directly studied; in fact, the relationship between child maltreatment and employment status in adulthood has rarely been examined (Tam, Zlotnick, \& Robertson, 2003; Tanaka et al., 2011; Thompson et al., 2012; Zielinski, 2009). Additionally, no long-term, longitudinal studies have examined the link between exposure to IPV and labour force participation (Tanaka et al., 2011).

Although there is a paucity of research that specifically examines exposure to IPV and employment variables, there are a few studies that consider child maltreatment and prospective socioeconomic variables. Child maltreatment, which includes exposure to IPV (CFCSA, 1996; Moss, 2004), is correlated with a variety of challenges that have been determined to be detrimental to employment outcomes (Hall \& Parker, 2010; Liptak, 2009). ${ }^{7}$ This section of the review identifies research endeavours that have investigated child maltreatment (including exposure to IPV) and employment outcomes in adulthood. Concurrently, encumbrances related to IPV and employment variables, including psychopathological problems, psychosocial difficulties, and functional deficits will be contemplated. ${ }^{8}$

Zielinski's (2009) study met the search and inclusion requirements for this review. The author studied the relationship between childhood experiences of maltreatment and definitively considered employment outcomes. Employing a prospective cohort design to analyze the longterm effects of child abuse and neglect, the author examined several indicators of socioeconomic well-being in adulthood. Nine dependent variables related to the participants' socioeconomic situation were evaluated; these measures included employment status, income levels, and health insurance coverage. The author used data from the National Comorbidity Survey (NCS). This psychiatric epidemiologic general population survey has been used previously to examine issues of socioeconomic well-being, child maltreatment, and family violence, as well as numerous forms of psychopathology. The complete sample size for this review was 5,004 with a mean age of 35.32. Importantly, in his literature review, Zielinski (2009) pointed out that, "nearly all of the studies that have examined this issue have suffered from significant methodological limitations" (p. 667) and subsequently addressed many of these challenges.

The results of the Zielinski (2009) study indicated that, when compared to individuals without maltreatment histories, adults who had "experienced maltreatment were twice as likely as non-maltreated adults to be unemployed at the time of survey" and " $80 \%$ more likely to have

\footnotetext{
${ }^{7}$ This paper is not intended to be a comprehensive literature review on common barriers to employment.

8 The complete text addresses many of these challenges in depth; this section considers the possible impact to employment outcomes.
} 
International Journal of Child, Youth and Family Studies (2014) 5(4): 493-587

In Harm's Way: A Special Issue on the Impacts and Costs of Witnessing Intimate Partner Violence

had someone in their household lose their job over the previous 12 months” (p. 671).

Furthermore, participants who reported maltreatment in childhood were more than twice as likely to fall below poverty levels, and twice as likely to live in the lowest quartile of income distribution. Maltreatment victims were also less likely to have health insurance coverage. Participants with histories of severe neglect were more likely to be employed, but were impacted by unemployment in the household (60\% in the past year) and were $90 \%$ more likely to fall below the poverty line. Zielinski's study provided more evidence for dual exposure theories (i.e., the "double whammy" effect) in the realm of employment: "adults who had experienced more than one type of maltreatment were at increased risk for employment difficulties” (p. 673).

Given the dearth of studies that fit the specific search terms of exposure to IPV and employment outcomes, the methodological limitations in this research area, and the possibility that similar variables are being examined using a variety of terms, the research parameters were expanded to include symptoms of trauma that are associated with IPV and employment outcomes. It is known that children who are exposed to IPV typically experience multiple traumas and other adversities (Evans et al., 2008; Herrenkohl et al., 2008; Lamers-Winkelman, Willemen, et al., 2012).

Related studies indicate that trauma in childhood may have deleterious effects on employment trajectories. Sansone, Leung, and Wiederman (2012) designed a project to study five types of childhood trauma and employment measures. Of the respondents who participated in the survey $(n=328)$, 43\% indicated that they had witnessed violence during childhood, $15 \%$ had experienced physical neglect, $44.8 \%$ had experienced emotional abuse, $25.9 \%$ had experienced physical abuse, 22.9\% had experienced sexual abuse, and 37.2\% denied having any experiences of childhood trauma. The questions related to employment queried the participants on their number of full-time jobs, percentage of time they had been employed full or part time, jobs that paid under the table, and being fired from a position.

Sansone et al.’s (2012) results suggest that, "trauma in childhood has varying and differential effects on employment in adulthood, depending on the type of trauma and the individual employment variable under study” (p. 678). Notably, witnessing violence was correlated with a greater likelihood of being fired from a job, and sexual abuse was associated with a higher number of total occupations and a greater likelihood of being fired. The other forms of childhood trauma garnered no statistically significant relationships with employment variables in this study. These findings differ from Zielinksi’s (2009) results.

Analyzing the link between traumatic experiences and consequent future expectations, Thompson et al. (2012) explored expectations surrounding academic and employment success, employment instability, and social instability. For adolescents, perceived expectations may influence behaviour and actual outcomes (e.g., increased sensation seeking and risk taking, lowered impulse control). Although exposure to family violence was not significantly correlated with future expectation domains in this study, maltreatment predicted low expectations in regard to academic and employment achievement and high expectations related to employment instability. The authors conclude that further research is required to determine how expectations in childhood and adolescence influence future functioning. 
International Journal of Child, Youth and Family Studies (2014) 5(4): 493-587

In Harm's Way: A Special Issue on the Impacts and Costs of Witnessing Intimate Partner Violence

In a Canadian study, Tanaka et al. (2011) examined the correlations between childhood physical and sexual abuse and employment outcomes. Although not necessarily related to exposure to IPV in childhood, these variables certainly fall under the domain of child maltreatment. Importantly, the authors made explicit efforts to overcome common limitations in this type of study. They used data from a more generalizable population, focused on both males and females, and examined the impact of more than one type of abuse. The study used data from the longitudinal Ontario Child Health Study, which were collected in 1983, 1987, and 2001. The final sample comprised 1,893 participants who had indicated experiences of childhood physical or sexual abuse. Data pertaining to employment variables were analyzed for 1,616 participants who were employed during the previous 12 months (defined by the Statistics Canada definition of labour force status). Importantly, variables from 1983 were included to control for childhood factors other than abuse. The study determined that adults who experienced severe physical abuse earned $29.6 \%$ less annual income compared to non-abused individuals. The authors indicate that this finding is "somewhat consistent with previous studies that have suggested a negative effect of child abuse on earnings" (p. 837). For employment variables, the authors found no significant associations with exposure to abuse, although the results indicated appreciable employment vulnerability for severely maltreated males. Tanaka et al. encouraged future researchers to consider other "potential factors that have a negative influence on earning and might be associated with exposure to child abuse, such as difficulty in maintaining employment, life stressors, and other psychological impairment including aggression, antisocial behavior, and substance use problems” (p. 838).

Barriers to employment. A multitude of researchers have revealed enduring detrimental consequences that are correlated with exposure to IPV (see Dauvergne \& Johnson, 2001; English et al., 2005; Herrenkohl \& Herrenkohl, 2007; Herrenkohl et al., 2008; Lamers-Winkelman, Willemen, et al., 2012; Meltzer et al., 2009). Many of these factors could contribute to employment deficits in adulthood: "barriers [to employment] stem from many circumstances, and they likely are multidimensional in terms of their impact and response to rehabilitation" (Johannesen, McGrew, Griss, \& Born, 2009, p. 298). Common obstacles to employment include poor work history and experience, insufficient education, lack of a support system, personal problems (e.g., financial, transportation), physical illness, and discrimination (Liptak, 2009; Sigurdsson, Ring, O'Reilly, \& Silverman, 2012). Barriers specifically related to psychological attributes include concentration challenges, psychosocial difficulties, emotional regulation deficits, stress management problems, substance use, and other essential impairments (Johannesen et al., 2009; Liptak, 2009; Sigurdsson et al., 2012). Importantly, psychological challenges can be problematic to resolve because "they have a direct impact on self-confidence and self-esteem, and can contribute to feelings of stress, anger, depression, and helplessness" (Liptak, 2009, p. 14).

\section{Conclusions: The Weight of the Evidence}

At the beginning of this review of the literature on the impact of exposure to intimate partner violence for children and youth, the perspective established was a developmental ecological approach, one in which any particular trajectory of a child's development emerges as 
International Journal of Child, Youth and Family Studies (2014) 5(4): 493-587

In Harm's Way: A Special Issue on the Impacts and Costs of Witnessing Intimate Partner Violence

a web of complex interactions between individual factors and environmental characteristics (Hertzman, 2012). This approach has proven to be useful, as it was speculated it might, in understanding the "multiple etiologies and sequelae of domestic violence" (Mohr \& Fantuzzo, 2000, p. 70). That web and those complex trajectories have crystallized more clearly through the review. Frequently the search found meaningful intersecting and interrelated impacts among those outcomes examined. After a brief synopsis of the initial foundational piece, what follows is a set of the major themes arising from the review, which serves as a roadmap through that complex landscape.

The review began with a consideration of the neurobiological impacts. A double-edged sword effect is said to arise from the reality that simply witnessing domestic discord can impact on the development of the main stress-regulatory system (that is, the hypothalamic-pituitaryadrenal axis and autonomic nervous system) such that the emerging stress system can lead to malfunctions in the neurochemical regulatory system. The subsequent cost occurs much later when the dysregulated physiological mechanisms may lead to a host of physical and mental sequelae. Unfortunately, because this manifestation may occur many years later, the direct association between the exposure to IPV and health issues, as well as behavioural and social adjustment problems, may remain obscured.

An important related neuropsychological finding points to the direction of integrating individual activity patterns in the sympathetic nervous system and parasympathetic nervous system into specific response profiles reflecting interactions between these two autonomic nervous system branches. Context-related ambiguity involved in interpretations of neurobiological response patterns was one of the most critical findings. Consistent with contextual mediation hypotheses (Belsky et al., 2007; Ellis \& Boyce, 2008, 2011; Steinberg \& Avenevoli, 2000), environmental factors seem to modulate the specific relationship between physiological activity patterns and health outcomes.

Thus the same pattern of neurobiological responses can serve both as a protective factor against, and as a risk factor for, pathways of pathological development under favourable and stressful conditions (e.g., IPV), respectively (Obradović et al., 2011). In other words, just because the developing brain is impacted by exposure to or witnessing parental conflicts does not necessarily mean those will result in negative impacts. It depends on the environmental context in which those experiences occur, whether positive and protective or negative and stressful. In that sense, the context directs the final response to emerge along a continuum of possible positive and negative outcomes. One example given from the review to illustrate this was the situation in which a child had witnessed intimate partner violence in the family as directed toward the mother, but because the mother was supportive and protective of the child, he/she did not appear subsequently to develop dysfunctional internalizing or externalizing behaviours.

The latter reality speaks to one theme that was common throughout our review. That is, the wonderment in these findings about why some of the children and youth, despite the experience of witnessing intimate partner violence apparently overcome the negativity to assume "normal" development and behaviour patterns. The answers to that question may assist in the 
International Journal of Child, Youth and Family Studies (2014) 5(4): 493-587

In Harm's Way: A Special Issue on the Impacts and Costs of Witnessing Intimate Partner Violence

development of successful preventive interventions as much as the answers to the question of what drives the negative impacts for children and youth in the first place.

What are clear, however, are the potentially quite debilitating effects on a significant proportion of children and youth who witness or are exposed to intimate partner violence. That outcome indeed constitutes the major common theme emerging from the review. This was true no matter which categories of interest were examined: neurobiology, physiology, health, illness, hospitalization, mental disorder, substance misuse, addiction, internalizing and externalizing problems, conduct disorder, behavioural issues, crime, delinquency, victimization, academic achievement, and employment. To have them examined together allowed for a comparison and mapping of the outcomes amongst them, and possible points of intersection and intervention.

Considering first then the issue of internalizing and externalizing problems, we find that although current research is still attempting to delineate specific etiologies and trajectories, witnessing IPV has also been clearly correlated with a multitude of internalizing and externalizing problems (Briggs-Gowan et al., 2010; Cosgrove et al., 2011; English et al., 2009; Ford et al., 2011; Slade, 2007). Inevitably, contemporary studies may portray fragmented descriptions of the consummate impact of IPV exposure and the proposed dichotomies of externalizing and internalizing symptomatology tend to result in rudimentary descriptions of complex problems (Cosgrove et al., 2011; Ford et al., 2011). The significant comorbidity of psychiatric disorders indicates that there are ongoing challenges for mental health diagnoses and subsequent research. For example, there is significant overlap between depressive symptoms and aggressive behaviour. On the other hand, the science continues to evolve and psychosocial models and recovery-focused research reveal some of the complexities inherent in this realm (Cosgrove et al., 2011; Slade, 2007).

Without the compounding effects of child maltreatment and abuse that often co-occur with family violence, exposure to IPV has a definitive detrimental impact on mental health outcomes in the life course of individuals who are impacted by IPV (Bayarri et al., 2011; Bogat et al., 2006; Bourassa, 2007; Gewirtz \& Edleson, 2007; Lamers-Winkelman, Willemen, et al., 2012). Although children who are threatened by high levels of IPV are at greater risk for developing emotional and behavioural problems, research confirms that children are affected by IPV, regardless of the degree of the exposure (Bayarri et al., 2011; Bourassa, 2007; Gewirtz \& Edleson, 2007; Lamers-Winkelman, Willemen, et al., 2012).

In discussing the various aspects and dimensions of the impacts of exposure of intimate partner violence on children and youth, we observed that these are not mutually exclusive. The notion of cascading effects informed our framework and analysis as it became ever more clearly evident that the individual categories of impacts were not only closely related to one another, but in a dynamic fashion influence each other in multiple and interconnected ways over time. The research that we have reviewed clearly shows that children who are exposed to intimate partner violence are at significant risk for lifelong negative outcomes. These outcomes have their beginnings in early exposure to intimate partner violence and the establishment of a "psychobiological fingerprint" (Boyce et al., 2001) and "psychological scars" (Saltzman et al., 2005) that can subsequently develop into internalizing and externalizing behaviours, conduct 
International Journal of Child, Youth and Family Studies (2014) 5(4): 493-587

In Harm's Way: A Special Issue on the Impacts and Costs of Witnessing Intimate Partner Violence

disorders, or addictions, and can, in turn, lead to crime and delinquency, victimization, academic dysfunction, and employment challenges. The impacts of exposure to intimate partner violence are certainly felt first by the individual children and youth who suffer these exposures; however, as the impacts are felt in the lives of these young people, they also are also felt in their life worlds: their early childhood education and care centres, schools, peer groups, community connections, places of employment, health care centres and hospitals, detention centres, and so forth. In the end, exposure to intimate partner violence is in no way merely an intimate experience. It is a toxic, harm-producing experience that cascades into all aspects of our shared social world.

\section{Limitations}

Despite advances in research on the subject of children's exposure to domestic violence since the early 1980s, a number of methodological problems remain. These include:

1. Lack of clarity concerning the definition of "exposure" to domestic violence;

2. Few standardized tools for assessing domestic violence exposure and child outcomes, and overreliance on some measures, such as the Conflict Tactics Scale (Straus, 1979; Straus et al., 1996);

3. Limited generalizability due to oversampling from shelter populations;

4. Small sample sizes and lack of comparison groups;

5. Overreliance on self-reporting, and mother-reported exposure and outcomes, instead of multiple informants;

6. Limited attention to confounding variables; and

7. Insufficient attention to the continuum of exposure and variations in children's involvement (Bair-Merritt et al., 2006; Chan \& Yeung, 2009; Evans et al., 2008; Fowler \& Chanmugam, 2007; Gewirtz \& Edleson, 2007; Holt et al., 2008; Kitzmann et al., 2003; Martin, 2002; Mohr et al., 2000; Sternberg, Lamb, et al., 2006; Wolfe et al., 2003).

Although the conclusions offered in this review seem to be grounded on a solid foundation, there are good reasons for exercising caution when interpreting the results. Most importantly, it is taken for granted that "exposure" to domestic violence has the same meaning across studies, but clear definitions and operational measurements of the construct run into difficulties (Holden, 2003). Accurate assessment of children's exposure to IPV is hampered by indirect retrospective measurement of vaguely defined terms like "exposure" to "domestic conflict” or "intimate partner violence” (Edleson et al., 2007). According to Edleson et al. (2007), the most often used - albeit imprecise - measurement technique relies on self-report formats inviting mothers and fathers to indicate how often their child witnessed (saw or heard) parental conflicts, suggesting that empirical criteria of exposure to IPV include children (a) not being the primary target of the violence, but (b) witnessing (seeing or hearing) the incident.

Much of the research on children's exposure to domestic violence relies on maternal or primary caregiver reports of IPV, and does not verify or corroborate these reports with those of children themselves, or with official documents such as medical records (e.g., Ackerson \& Subramanian, 2009; Bair-Merritt et al., 2008; Hasselmann \& Reichenheim, 2006; Olofsson et al., 
International Journal of Child, Youth and Family Studies (2014) 5(4): 493-587

In Harm's Way: A Special Issue on the Impacts and Costs of Witnessing Intimate Partner Violence

2011; Silverman et al., 2011; Sobkoviak et al., 2012). A related limitation is that retrospective reporting is subject to recall bias and possible underreporting, particularly when participants are asked to report on violence they experienced many years in the past (Anda et al., 2006; Brown et al., 2009; Dube et al., 2009; Olofsson et al., 2011; Ramiro et al., 2010; Silverman et al., 2011; Sobkoviak et al., 2012). Finally, due to the sensitive nature of questions about IPV, including physical, emotional, and sexual violence, participants may not be forthcoming about their experiences (Brown et al., 2010). This is particularly true in societies or cultures where social stigma related to domestic and sexual violence against women is high (Sobkoviak et al., 2012).

A lack of consensus on the definition of "exposure" and a lack of specificity in measurement continue to present limitations for this field of research. For example, in the area of health impacts, the frequency and/or severity of health outcomes (e.g., headaches) are not always taken into account. As well, the majority of research in this area indicates an association, rather than a causal relationship between the incidence of IPV against women and negative outcomes for children and youth (Åsling-Monemi et al., 2008; Brady, 2008; Sobkoviak et al., 2012). Finally, in the physical health arena, research conducted in developing countries on the subject of nutritional status and mortality may have limited generalizability.

With respect to mental health outcomes, Camacho et al. (2012) outlined a number of methodological problems that plagued early research in the area of IPV exposure. Many studies relied on clinical samples of children and cross-sectional, retrospective designs. As such, these findings may not be generalizable to a larger population and some of the measured effects of IPV may be attributed to recent events and residing in shelters (Cosgrove et al., 2011; Manion, 2010; Slade, 2007). Furthermore, the lack of longitudinal data makes it impossible to determine whether some individuals may develop mental health problems in later years (Ford et al., 2011). Contemporary research has made substantial efforts to address these limitations to improve reliability and validity of the study results. Additionally, current projects are beginning to examine possible moderators and mediators of IPV exposure (Camacho et al., 2012; Spilsbury et al., 2008). While much of the existent research on IPV exposure and mental well-being have focused on children aged 5 to 12, and have been limited to a single encounter in the lifespan of the individual, studies have revealed the same detrimental results when examining adult outcomes (Howells \& Rosenbaum, 2008).

Presently, scientific investigation on exposure to family violence and other family-related traumas remains limited (Crusto et al., 2010). Further study in the realm of IPV exposure and mental health challenges is required to determine possible adjustment trajectories (Spilsbury et al., 2008). Unfortunately, the lack of research funding and appropriate services for youth in Canada compounds the problem, and may leave youth with lifelong encumbrances (Manion, 2010; Mental Health Commission of Canada, 2012). The detrimental consequences of IPV exposure may linger throughout the lifespan of the individual and, if recognized, may require extensive treatment. Regrettably, many of the subtle effects may not be diagnosable and remain untreated (Bayarri et al., 2011; Gewirtz \& Medhanie, 2008; Moylan et al., 2010).

Exposure to IPV is associated with the presence of externalized and internalized symptoms in children, and a multitude of other mental health disorders. Few studies extrapolate 
International Journal of Child, Youth and Family Studies (2014) 5(4): 493-587

In Harm's Way: A Special Issue on the Impacts and Costs of Witnessing Intimate Partner Violence

potential moderating and mediating variables of IPV exposure, and while research suggests a multitude of risks, some children are functioning well after IPV exposure (Ford et al., 2011; Sternberg, Lamb, et al., 2006). New knowledge could enhance treatment modalities and, as a result, possibly improve psychopathological outcomes and create lasting functional improvements (Bourassa, 2007; Crusto et al., 2010). Presently, little is known about long-term trajectories of children who have been exposed to IPV and what variables may enhance their life course paths (Ford et al., 2011; Sternberg, Lamb, et al., 2006). However, it is clear that violence in the home contributes to an environment that is frightening, confusing, and lacking in security and safety, and that IPV exposure places a substantial burden on the mental health and wellbeing of individuals, families, and communities (Bayarri et al., 2011; Lamers-Winkelman, Willemen, et al., 2012; Mental Health Commission of Canada, 2012; World Health Organization [WHO], 2013). 
International Journal of Child, Youth and Family Studies (2014) 5(4): 493-587

In Harm's Way: A Special Issue on the Impacts and Costs of Witnessing Intimate Partner

Violence

\section{References}

Achenbach, T. M. (1991). Manual for the youth self-report and 1991 profile. Burlington, VT: University of Vermont, Department of Psychiatry.

Achenbach, T. M., \& Edelbrock, C. S. (1983). Manual for the child behavior checklist and revised child behavior profile. Burlington, VT: University of Vermont, Deptartment of Psychiatry.

Ackerson, L. K., \& Subramanian, S. V. (2008). Domestic violence and chronic malnutrition among women and children in India. American Journal of Epidemiology, 167(10), 11881196. doi: 10.1093/aje/kwn049

Ackerson, L. K., \& Subramanian, S. (2009). Intimate partner violence and death among infants and children in India. Pediatrics, 124(5), e878-e889. doi: 10.1542/peds.2009-0524

Adams, C. M. (2006). The consequences of witnessing family violence on children and implications for family counselors. The Family Journal, 14(4), 334-341. doi: $10.1177 / 1066480706290342$

Adams, D. (2009). Predisposing childhood factors for men who kill their intimate partners. Victims and Offenders, 4(3), 215-229. doi: 10.1080/15564880903048479

Afifi, T. O. (2011). Child maltreatment in Canada: An understudied public health problem. Canadian Journal of Public Health, 102(6), 459-461. Retrieved from http://journal.cpha.ca/

Alexander, P. C. (2011). Childhood maltreatment, intimate partner violence, work interference and women's employment. Journal of Family Violence, 26(4), 255-261. doi: 10.1007/s10896-011-9361-9

American Psychiatric Association. (2013). Diagnostic and statistical manual of mental disorders (5th ed.). Arlington, VA: Author.

Anda, R. F., Brown, D. W., Dube, S. R., Bremner, J. D., Felitti, V. J., \& Giles, W. H. (2008). Adverse childhood experiences and chronic obstructive pulmonary disease in adults. American Journal of Preventive Medicine, 34(5), 396-403. doi: 10.1016/j.amepre.2008.02.002

Anda, R. F., Felitti, V. J., Bremner, J. D., Walker, J. D., Whitfield, C., Perry, B. D.,...Giles, W. H. (2006). The enduring effects of abuse and related adverse experiences in childhood. European Archives of Psychiatry and Clinical Neuroscience, 256(3), 174-186. doi: 10.1007/s00406-005-0624-4 
International Journal of Child, Youth and Family Studies (2014) 5(4): 493-587

In Harm's Way: A Special Issue on the Impacts and Costs of Witnessing Intimate Partner

Violence

Anda, R. F., Tietjen, G., Schulman, E., Felitti, V., \& Croft, J. (2010). Adverse childhood experiences and frequent headaches in adults. Headache: The Journal of Head and Face Pain, 50(9), 1473-1481. doi: 10.1111/j.1526-4610.2010.01756.x

Andersen, S. L., \& Teicher, M. H. (2009). Desperately driven and no brakes: Developmental stress exposure and subsequent risk for substance abuse. Neuroscience \& Biobehavioral Reviews, 33(4), 516-524. doi: 10.1016/j.neubiorev.2008.09.009

Anderson, K. M., \& Bang, E.-J. (2012). Assessing PTSD and resilience for females who during childhood were exposed to domestic violence. Child \& Family Social Work, 17(1), 55-65. doi: 10.1111/j.1365-2206.2011.00772.x

Åsling-Monemi, K., Naved, R. T., \& Persson, L. Å. (2008). Violence against women and the risk of under-five mortality: Analysis of community-based data from rural Bangladesh. Acta Paediatrica, 97(2), 226-232. doi: 10.1111/j.1651-2227.2007.00597.x

Bair-Merritt, M. H., Blackstone, M., \& Feudtner, C. (2006). Physical health outcomes of childhood exposure to intimate partner violence: A systematic review. Pediatrics, 117(2), e278-e290. doi: 10.1542/peds.2005-1473

Bair-Merritt, M. H., Feudtner, C., Localio, A. R., Feinstein, J. A., Rubin, D., \& Holmes, W. C. (2008). Health care use of children whose female caregivers have intimate partner violence histories. Archives of Pediatrics \& Adolescent Medicine, 162(2), 134-139. doi: 10.1001/archpediatrics.2007.32

Bair-Merritt, M. H., Johnson, S. B., Okelo, S., \& Page, G. (2012). Intimate partner violence exposure, salivary cortisol, and childhood asthma. Child Abuse \& Neglect, 36(7), 596601. doi: 10.1016/j.chiabu.2011.12.002

Baker, L., \& Cunningham, A. (2009). Inter-parental violence: The pre-schooler's perspective and the educator's role. Early Childhood Education Journal, 37(3), 199-207. doi: 10.1007/s10643-009-0342-z

Bakermans-Kranenburg, M. J., \& van IJzendoorn, M. H. (2011). Differential susceptibility to rearing environment depending on dopamine-related genes: New evidence and a metaanalysis. Development and Psychopathology, 23(1), 39-52. doi: 10.1017/S0954579410000635

Bauer, N. S., Gilbert, A. L., Carroll, A. E., \& Downs, S. M. (2013). Associations of early exposure to intimate partner violence and parental depression with subsequent mental health outcomes. JAMA pediatrics, 167(4), 341-347. doi: 10.1001/jamapediatrics.2013.780. 
International Journal of Child, Youth and Family Studies (2014) 5(4): 493-587

In Harm's Way: A Special Issue on the Impacts and Costs of Witnessing Intimate Partner

Violence

Bauer, N. S., Herrenkohl, T. I., Lozano, P., Rivara, F. P., Hill, K. G., \& Hawkins, J. D. (2006). Childhood bullying involvement and exposure to intimate partner violence. Pediatrics, 118(2), e235-e242. doi: 10.1542/peds.2005-2509

Bayarri, E., Ezpeleta, L., \& Granero, R. (2011). Exposure to intimate partner violence, psychopathology, and functional impairment in children and adolescents: Moderator effect of sex and age. Journal of Family Violence, 26(7), 535-543. doi: 10.1007/s10896011-9390-4

BC Society of Transition Houses. (2011). Report on violence against women, mental health and substance use. Vancouver: Author.

Becker, K. B., \& McCloskey, L. A. (2002). Attention and conduct problems in children exposed to family violence. American Journal of Orthopsychiatry, 72(1), 83-91. doi: 10.1037/0002-9432.72.1.83

Becker-Blease, K. A., \& Freyd, J. J. (2008). A preliminary study of ADHD symptoms and correlates: Do abused children differ from nonabused children? Journal of Aggression, Maltreatment \& Trauma, 17(1), 133-140. doi: 10.1080/10926770802250736

Bedi, G., \& Goddard, C. (2007). Intimate partner violence: What are the impacts on children? Australian Psychologist, 42(1), 66-77. doi: 10.1080/00050060600726296

Begle, A. M., Hanson, R. F., Danielson, C. K., McCart, M. R., Ruggiero, K. J., Amstadter, A. B., .... Kilpatrick, D. G. (2011). Longitudinal pathways of victimization, substance use, and delinquency: Findings from the National Survey of Adolescents. Addictive Behaviors, 36(7), 682-689. doi: 10.1016/j.addbeh.2010.12.026

Belsky, J., Bakermans-Kranenburg, M. J., \& van IJzendoorn, M. H. (2007). For better and for worse differential susceptibility to environmental influences. Current Directions in Psychological Science, 16(6), 300-304. doi: 10.1111/j.1467-8721.2007.00525.x

Black, T., Trocmé, N., Fallon, B., \& MacLaurin, B. (2008). The Canadian child welfare system response to exposure to domestic violence investigations. Child Abuse \& Neglect, 32(3), 393-404. doi: 10.1016/j.chiabu.2007.10.002

Bogat, G. A., DeJonghe, E., Levendosky, A. A., Davidson, W. S., \& von Eye, A. (2006). Trauma symptoms among infants exposed to intimate partner violence. Child Abuse \& Neglect, 30(2), 109-125. doi: 10.1016/j.chiabu.2005.09.002

Bos, K. J., Fox, N., Zeanah, C. H., \& Nelson, C. A. (2009). Effects of early psychosocial deprivation on the development of memory and executive function. Frontiers in Behavioral Neuroscience, 3, 1-7. doi: 10.3389/neuro.08.016.2009 
International Journal of Child, Youth and Family Studies (2014) 5(4): 493-587

In Harm's Way: A Special Issue on the Impacts and Costs of Witnessing Intimate Partner

Violence

Bos, K., Zeanah, C. H., Fox, N. A., Drury, S. S., McLaughlin, K. A., \& Nelson, C. A. (2011). Psychiatric outcomes in young children with a history of institutionalization. Harvard Review of Psychiatry, 19(1), 15-24. doi: 10.3109/10673229.2011.549773

Bourassa, C. (2007). Co-occurrence of interparental violence and child physical abuse and it's [sic] effect on the adolescents' behavior. Journal of Family Violence, 22(8), 691-701. doi: 10.1007/s10896-007-9117-8

Boyce, W. T., Quas, J., Alkon, A., Smider, N. A., Essex, M. J., \& Kupfer, D. J. (2001). Autonomic reactivity and psychopathology in middle childhood. British Journal of Psychiatry, 179(2), 144-150. doi: 10.1192/bjp.179.2.144

Boyce, W. T., Sokolowski, M. B., \& Robinson, G. E. (2012). Toward a new biology of social adversity. Proceedings of the National Academy of Sciences, 109(Supplement 2), 1714317148. doi: 10.1073/pnas.1121264109

Brady, S. S. (2008). Lifetime family violence exposure is associated with current symptoms of eating disorders among both young men and women. Journal of Traumatic Stress, 21(3), 347-351. doi: 10.1002/jts.20335

Bremner, J. D., Randall, P., Scott, T. M., Capelli, S., Delaney, R., McCarthy, G., \& Charney, D. S. (1995). Deficits in short-term memory in adult survivors of childhood abuse. Psychiatry Research, 59(1), 97-107. doi: 10.1016/0165-1781(95)02800-5

Briggs-Gowan, M. J., Carter, A. S., Clark, R., Augustyn, M., McCarthy, K. J., \& Ford, J. D. (2010). Exposure to potentially traumatic events in early childhood: Differential links to emergent psychopathology. Journal of Child Psychology and Psychiatry, 51(10), 11321140. doi: 10.1111/j.1469-7610.2010.02256.x

Brown, D. W., Anda, R. F., Felitti, V. J., Edwards, V. J., Malarcher, A. M., Croft, J. B., \& Giles, W. H. (2010). Adverse childhood experiences are associated with the risk of lung cancer: A prospective cohort study. BMC Public Health, 10(20), 1-12. doi: 10.1186/1471-2458$10-20$

Brown, D. W., Anda, R. F., Tiemeier, H., Felitti, V. J., Edwards, V. J., Croft, J. B., \& Giles, W. H. (2009). Adverse childhood experiences and the risk of premature mortality. American Journal of Preventive Medicine, 37(5), 389-396. doi: 10.1016/j.amepre.2009.06.021

Browning, K., Thornberry, T., \& Porter, P. (1999). Highlights of findings from the Rochester youth development study. Washington, DC: US Department of Justice, Office of Juvenile Justice and Delinquency Prevention.

Buckley, H., Holt, S., \& Whelan, S. (2007). Listen to me! Children's experiences of domestic violence. Child Abuse Review, 16(5), 296-310. doi: 10.1002/car.995 
International Journal of Child, Youth and Family Studies (2014) 5(4): 493-587

In Harm's Way: A Special Issue on the Impacts and Costs of Witnessing Intimate Partner

Violence

Buehler, C., Anthony, C., Krishnakumar, A., Stone, G., Gerard, J., \& Pemberton, S. (1997). Interparental conflict and youth problem behaviors: A meta-analysis. Journal of Child and Family Studies, 6(2), 233-247. doi: 10.1023/A:1025006909538

Busch, A. L., \& Lieberman, A. F. (2010). Mothers' adult attachment interview ratings predict preschool children's IQ following domestic violence exposure. Attachment \& Human Development, 12(6), 505-527. doi: 10.1080/14616734.2010.504542

Butchart, A., Harvey, A. P., Mian, M., \& Furniss, T. (2006). Preventing child maltreatment: A guide to taking action and generating evidence. Geneva: World Health Organization.

Byrne, D., \& Taylor, B. (2007). Children at risk from domestic violence and their educational attainment: Perspectives of education welfare officers, social workers and teachers. Child Care in Practice, 13(3), 185-201. doi: 10.1080/13575270701353465

Caldeira, V., \& Woodin, E. M. (2012). Childhood exposure to aggression and adult relationship functioning: Depression and antisocial behavior as mediators. Journal of Family Violence, 27(7), 687-696. doi: 10.1007/s10896-012-9453-1

Camacho, K., Ehrensaft, M. K., \& Cohen, P. (2012). Exposure to intimate partner violence, peer relations, and risk for internalizing behaviors: A prospective longitudinal study. Journal of Interpersonal Violence, 27(1), 125-141. doi: 10.1177/0886260511416474

Canadian Alcohol and Drug Use Monitoring Survey (CADUMS). (2009). Highlights report. Ottawa: Health Canada. Retrieved from http://www.hc-sc.gc.ca/hc-ps/drugsdrogues/stat/index-eng.php

Canadian Association of Social Workers. (2013). CASW - Acts: The role of social work in mental health. Ottawa: Author. Retrieved from http://www.hc-sc.gc.ca/hc-ps/drugsdrogues/stat/index-eng.php

Carlson, B. E. (1984). Children's observations of interparental violence. In A. R. Roberts (Ed.), Battered women and their families (pp. 147-167). New York: Springer Publishing Company.

Carpenter, G. L., \& Stacks, A. M. (2009). Developmental effects of exposure to intimate partner violence in early childhood: A review of the literature. Children and Youth Services Review, 31(8), 831-839. doi: 10.1016/j.childyouth.2009.03.005

Carrell, S. E., \& Hoekstra, M. L. (2010). Externalities in the classroom: How children exposed to domestic violence affect everyone's kids. American Economic Journal: Applied Economics, 2(1), 211-228. doi: 10.1257/app.2.1.211

Centers for Disease Control and Prevention. (2008). Youth risk behavior surveillance: United States, 2008. Atlanta, GA: Author. 
International Journal of Child, Youth and Family Studies (2014) 5(4): 493-587

In Harm's Way: A Special Issue on the Impacts and Costs of Witnessing Intimate Partner

Violence

Centers for Disease Control and Prevention. (2012). Alcohol and public health fact sheets: Binge drinking. Atlanta, GA: Author.

Chan, Y.-C., \& Yeung, J. W.-K. (2009). Children living with violence within the family and its sequel: A meta-analysis from 1995-2006. Aggression and Violent Behavior, 14(5), 313322. doi: 10.1016/j.avb.2009.04.001

Chesney-Lind, M., \& Jones, N. (2010). Fighting for girls: New perspectives on gender and violence. New York: State University of New York Press.

Child, Family, and Community Service Act [CFCSA], R.S.B.C. 1996, c.46, online: BC Laws: EPL Libraries http://www.bclaws.ca/EPLibraries/bclaws_new/document/ID/freeside/00_96046_01 section14.

Choi, J., Jeong, B., Polcari, A., Rohan, M. L., \& Teicher, M. H. (2012). Reduced fractional anisotropy in the visual limbic pathway of young adults witnessing domestic violence in childhood. Neuroimage, 59(2), 1071-1079. doi: 10.1016/j.neuroimage.2011.09.033

Chrisler, J. C., \& Ferguson, S. (2006). Violence against women as a public health issue. Annals of the New York Academy of Sciences, 1087(1), 235-249. doi: 0.1196/annals.1385.009

Cicchetti, D., \& Rogosch, F. A. (1996). Equifinality and multifinality in developmental psychopathology. Development and Psychopathology, 8, 597-600. doi: 10.1017/S0954579400007318

Cipriano, E. A., Skowron, E. A., \& Gatzke-Kopp, L. M. (2011). Preschool children’s cardiac reactivity moderates relations between exposure to family violence and emotional adjustment. Child Maltreatment, 16(3), 205-215. doi: 10.1177/1077559511408887

Cisler, J. M., Begle, A. M., Amstadter, A. B., Resnick, H. S., Danielson, C. K., Saunders, B. E., \& Kilpatrick, D. G. (2012). Exposure to interpersonal violence and risk for PTSD, depression, delinquency, and binge drinking among adolescents: Data from the NSA-R. Journal of Traumatic Stress, 25(1), 33-40. doi: 10.1002/jts.21672

Clark, D. B., Bukstein, O., \& Cornelius, J. (2002). Alcohol use disorders in adolescents. Pediatric Drugs, 4(8), 493-502. doi: 1174-5878/02/0008-0493

Coohey, C., Renner, L. M., Hua, L., Zhang, Y. J., \& Whitney, S. D. (2011). Academic achievement despite child maltreatment: A longitudinal study. Child Abuse \& Neglect, 35(9), 688-699. doi: 10.1016/j.chiabu.2011.05.009

Cosgrove, V. E., Rhee, S. H., Gelhorn, H. L., Boeldt, D., Corley, R. C., Ehringer, M. A., ... Hewitt, J. K. (2011). Structure and etiology of co-occurring internalizing and 
International Journal of Child, Youth and Family Studies (2014) 5(4): 493-587

In Harm's Way: A Special Issue on the Impacts and Costs of Witnessing Intimate Partner

Violence

externalizing disorders in adolescents. Journal of Abnormal Child Psychology, 39(1), 109-123. doi: 10.1007/s10802-010-9444-8

Covey, H. C., Menard, S., \& Franzese, R. J. (2013). Effects of adolescent physical abuse, exposure to neighborhood violence, and witnessing parental violence on adult socioeconomic status. Child maltreatment, 18(2), 85-97. doi: 10.1177/1077559513477914

Crusto, C. A., Whitson, M. L., Walling, S. M., Feinn, R., Friedman, S. R., Reynolds, J., ... Kaufman, J. S. (2010). Posttraumatic stress among young urban children exposed to family violence and other potentially traumatic events. Journal of Traumatic Stress, 23(6), 716-724. doi: 10.1002/jts.20590

Cummings, E. M., El-Sheikh, M., Kouros, C. D., \& Buckhalt, J. A. (2009). Children and violence: The role of children's regulation in the marital aggression-child adjustment link. Clinical Child and Family Psychology Review, 12(1), 3-15. doi: 10.1007/s10567-0090042-7

Cunningham, A., \& Baker, L. (2004). What about me! Seeking to understand a child's view of violence in the family. London, ON: Centre for Children \& Families in the Justice System.

Currie, J., \& Widom, C. S. (2010). Long-term consequences of child abuse and neglect on adult economic well-being. Child Maltreatment, 15(2), 111-120. doi: 10.1177/1077559509355316

Danese, A., \& McEwen, B. S. (2012). Adverse childhood experiences, allostasis, allostatic load, and age-related disease. Physiology \& Behavior, 106(1), 29-39. doi: 10.1016/j.physbeh.2011.08.019

Dauvergne, M., \& Johnson, H. (2001). Children witnessing family violence. Ottawa: Canadian Centre for Justice Statistics.

Davies, P. T., Sturge-Apple, M. L., \& Cicchetti, D. (2011). Interparental aggression and children's adrenocortical reactivity: Testing an evolutionary model of allostatic load. Development and Psychopathology, 23(03), 801-814. doi: 10.1017/S0954579411000319

Davies, P. T., Sturge-Apple, M. L., Cicchetti, D., \& Cummings, E. M. (2007). The role of child adrenocortical functioning in pathways between interparental conflict and child maladjustment. Developmental psychology, 43(4), 918-930. doi: 10.1037/00121649.43.4.918

Davies, P. T., Sturge-Apple, M. L., Cicchetti, D., \& Cummings, E. M. (2008). Adrenocortical underpinnings of children's psychological reactivity to interparental conflict. Child Development, 79(6), 1693-1706. doi: 10.1111/j.1467-8624.2008.01219.x 
International Journal of Child, Youth and Family Studies (2014) 5(4): 493-587

In Harm's Way: A Special Issue on the Impacts and Costs of Witnessing Intimate Partner

Violence

Davies, P. T., Sturge-Apple, M. L., Cicchetti, D., Manning, L. G., \& Vonhold, S. E. (2012). Pathways and processes of risk in associations among maternal antisocial personality symptoms, interparental aggression, and preschooler's psychopathology. Development and Psychopathology, 24, 807-823. doi: 10.1017/S0954579412000387

Davies, P. T., Sturge-Apple, M. L., Cicchetti, D., Manning, L. G., \& Zale, E. (2009). Children's patterns of emotional reactivity to conflict as explanatory mechanisms in links between interpartner aggression and child physiological functioning. Journal of Child Psychology and Psychiatry, 50(11), 1384-1391. doi: 10.1111/j.1469-7610.2009.02154.x

DeJonghe, E. S., von Eye, A., Bogat, G. A., \& Levendosky, A. A. (2011). Does witnessing intimate partner violence contribute to toddlers' internalizing and externalizing behaviors? Applied Developmental Science, 15(3), 129-139. doi: 10.1080/10888691.2011.587713

Denver, R. J., \& Crespi, E. J. (2006). Stress hormones and human developmental plasticity lessons from tadpoles. NeoReviews, 7(4), e183-e188. doi: 10.1542/neo.7-4-e183

DePrince, A. P., Chu, A. T., \& Combs, M. D. (2008). Trauma-related predictors of deontic reasoning: A pilot study in a community sample of children. Child Abuse \& Neglect, 32(7), 732-737. doi: 0.1016/j.chiabu.2007.10.006

Douglas, K. R., Chan, G., Gelernter, J., Arias, A. J., Anton, R. F., Weiss, R. D., ... Kranzler, H. R. (2010). Adverse childhood events as risk factors for substance dependence: Partial mediation by mood and anxiety disorders. Addictive Behaviors, 35(1), 7-13. doi: 10.1016/j.addbeh.2009.07.004

Dube, S. R., Fairweather, D., Pearson, W. S., Felitti, V. J., Anda, R. F., \& Croft, J. B. (2009). Cumulative childhood stress and autoimmune diseases in adults. Psychosomatic Medicine, 71(2), 243-250. doi: 10.1097/PSY.0b013e3181907888

Duncan, A., Thomas, J. C., \& Miller, C. (2005). Significance of family risk factors in development of childhood animal cruelty in adolescent boys with conduct problems. Journal of Family Violence, 20(4), 235-239. doi: 10.1007/s10896-005-5987-9

Edleson, J. L., Ellerton, A. L., Seagren, E. A., Kirchberg, S. L., Schmidt, S. O., \& Ambrose, A. T. (2007). Assessing child exposure to adult domestic violence. Children and Youth Services Review, 29(7), 961-971. doi: 10.1016/j.childyouth.2006.12.009

Ehrensaft, M. K., \& Cohen, P. (2012). Contribution of family violence to the intergenerational transmission of externalizing behavior. Prevention Science, 13(4), 370-383. doi: 10.1007/s11121-011-0223-8 
International Journal of Child, Youth and Family Studies (2014) 5(4): 493-587

In Harm's Way: A Special Issue on the Impacts and Costs of Witnessing Intimate Partner

Violence

El-Sheikh, M., \& Erath, S. A. (2011). Family conflict, autonomic nervous system functioning, and child adaptation: State of the science and future directions. Development and Psychopathology, 23(02), 703-721. doi: 10.1017/S0954579411000034

El-Sheikh, M., \& Hinnant, J. B. (2011). Marital conflict, respiratory sinus arrhythmia, and allostatic load: Interrelations and associations with the development of children's externalizing behavior. Development and Psychopathology, 23(3), 815-829. doi: 10.1017/S0954579411000320

El-Sheikh, M., Hinnant, J. B., \& Erath, S. (2011). Developmental trajectories of delinquency symptoms in childhood: The role of marital conflict and autonomic nervous system activity. Journal of Abnormal Psychology, 120(1), 16-32. doi: 10.1037/a0020626

El-Sheikh, M., Keiley, M., Erath, S., \& Dyer, W. J. (2013). Marital conflict and growth in children's internalizing symptoms: The role of autonomic nervous system activity. Developmental Psychology, 49(1), 92-108. doi: 10.1037/a0027703

El-Sheikh, M., Keller, P. S., \& Erath, S. A. (2007). Marital conflict and risk for child maladjustment over time: Skin conductance level reactivity as a vulnerability factor. Journal of Abnormal Child Psychology, 35(5), 715-727. doi:10.1007/s10802-007-9127-2

El-Sheikh, M., Kouros, C. D., Erath, S., Cummings, E. M., Keller, P., \& Staton, L. (2009). Marital conflict and children's externalizing behavior: Pathways involving interactions between parasympathetic and sympathetic nervous system activity. Monographs of the Society for Research in Child Development, 74(1), vii-79. doi: 10.1111/j.15405834.2009.00501.x

El-Sheikh, M., \& Whitson, S. A. (2006). Longitudinal relations between marital conflict and child adjustment: Vagal regulation as a protective factor. Journal of Family Psychology, 20(1), 30-39. doi: 10.1037/0893-3200.20.1.30

Ellis, B. J., \& Boyce, W. T. (2008). Biological sensitivity to context. Current Directions in Psychological Science, 17(3), 183-187. doi: 10.1111/j.1467-8721.2008.00571.x

Ellis, B. J., \& Boyce, W. T. (2011). Differential susceptibility to the environment: Toward an understanding of sensitivity to developmental experiences and context. Development and Psychopathology, 23(1), 1-5. doi: 10.1017/S095457941000060X

Ellonen, N., Piispa, M., Peltonen, K., \& Oranen, M. (2013). Exposure to parental violence and outcomes of child psychosocial adjustment. Violence and victims, 28(1), 3-15. doi: 10.1891/0886-6708.28.1.3

Emery, C. R. (2011). Controlling for selection effects in the relationship between child behavior problems and exposure to intimate partner violence. Journal of Interpersonal Violence, 26(8), 1541-1558. doi: 10.1177/0886260510370597 
International Journal of Child, Youth and Family Studies (2014) 5(4): 493-587

In Harm's Way: A Special Issue on the Impacts and Costs of Witnessing Intimate Partner

Violence

Engert, V., Efanov, S. I., Dedovic, K., Duchesne, A., Dagher, A., \& Pruessner, J. C. (2010). Perceived early-life maternal care and the cortisol response to repeated psychosocial stress. Journal of Psychiatry \& Neuroscience: JPN, 35(6), 370-377. doi: 10.1503/jpn.100022

English, D. J., Graham, J. C., Newton, R. R., Lewis, T. L., Thompson, R., Kotch, J. B., \& Weisbart, C. (2009). At-risk and maltreated children exposed to intimate partner aggression/violence what the conflict looks like and its relationship to child outcomes. Child Maltreatment, 14(2), 157-171. doi: 10.1177/1077559508326287

English, D. J., Upadhyaya, M. P., Litrownik, A. J., Marshall, J. M., Runyan, D. K., Graham, J. C., \& Dubowitz, H. (2005). Maltreatment's wake: The relationship of maltreatment dimensions to child outcomes. Child Abuse \& Neglect, 29(5), 597-619. doi: 10.1016/j.chiabu.2004.12.008

Enoch, M.-A. (2011). The role of early life stress as a predictor for alcohol and drug dependence. Psychopharmacology, 214(1), 17-31. doi: 10.1007/s00213-010-1916-6

Essex, M. J., Thomas Boyce, W., Hertzman, C., Lam, L. L., Armstrong, J. M., Neumann, S., \& Kobor, M. S. (2013). Epigenetic vestiges of early developmental adversity: Childhood stress exposure and DNA methylation in adolescence. Child Development, 84(1), 58-75. doi: 10.1111/j.1467-8624.2011.01641.x

Evans, S. E., Davies, C., \& DiLillo, D. (2008). Exposure to domestic violence: A meta-analysis of child and adolescent outcomes. Aggression and Violent Behavior, 13(2), 131-140. doi: 10.1016/j.avb.2008.02.005

Fantuzzo, J. W., \& Lindquist, C. U. (1989). The effects of observing conjugal violence on children: A review and analysis of research methodology. Journal of Family Violence, 4(1), 77-94. doi: 10.1007/BF00985658

Fergusson, D. M., Boden, J. M., \& Horwood, L. J. (2008a). The developmental antecedents of illicit drug use: Evidence from a 25-year longitudinal study. Drug and Alcohol Dependence, 96(1), 165-177. doi: 10.1016/j.drugalcdep.2008.03.003

Fergusson, D. M., Boden, J. M., \& Horwood, L. J. (2008b). Developmental antecedents of interpartner violence in a New Zealand birth cohort. Journal of Family Violence, 23(8), 737-753. doi: 10.1007/s10896-008-9199-y

Fisher, W. H., \& Grudzinskas, Jr., A. J. (2010). Crisis intervention teams as the solution to managing crises involving persons with serious psychiatric illnesses: Does one size fit all? Journal of Police Crisis Negotiations, 10(1/2), 58-71. doi:

10.1080/15332581003757263 
International Journal of Child, Youth and Family Studies (2014) 5(4): 493-587

In Harm's Way: A Special Issue on the Impacts and Costs of Witnessing Intimate Partner

Violence

Ford, J. D., Gagnon, K., Connor, D. F., \& Pearson, G. (2011). History of interpersonal violence, abuse, and nonvictimization trauma and severity of psychiatric symptoms among children in outpatient psychiatric treatment. Journal of Interpersonal Violence, 26(16), 3316-3337. doi: $10.1177 / 0886260510393009$

Fowler, D. N., \& Chanmugam, A. (2007). A critical review of quantitative analyses of children exposed to domestic violence: Lessons for practice and research. Brief Treatment and Crisis Intervention, 7(4), 322-344. doi: 10.1093/brief-treatment/mhm019

Fredland, N. M., Campbell, J. C., \& Han, H. (2008). Effect of violence exposure on health outcomes among young urban adolescents. Nursing Research, 57(3), 157-165. doi: 10.1097/01.NNR.0000319493.21628.c6

Garcia-Moreno, C., \& Watts, C. (2011). Violence against women: An urgent public health priority. Bulletin of the World Health Organization, 89(1), 2-3. doi:

10.2471/BLT.10.085217

Gavazzi, S. M., Lim, J.-Y., Yarcheck, C. M., Bostic, J. M., \& Scheer, S. D. (2008). The impact of gender and family processes on mental health and substance use issues in a sample of court-involved female and male adolescents. Journal of Youth and Adolescence, 37(9), 1071-1084. doi: 10.1007/s10964-008-9306-7

Gavazzi, S. M., Slade, D., Buettner, C. K., Partridge, C., Yarcheck, C. M., \& Andrews, D. W. (2003). Toward conceptual development and empirical measurement of global risk indicators in the lives of court-involved youth. Psychological Reports, 92(2), 599-615. doi: 10.2466/pr0.2003.92.2.599

Gewirtz, A., \& Edleson, J. (2004). Young children’s exposure to adult domestic violence: Toward a developmental risk and resilience framework for research and intervention Early childhood, domestic violence, and poverty: Helping young children and their families (Vol. 6). Iowa City, IA: University of Iowa.

Gewirtz, A. H., \& Edleson, J. L. (2007). Young children's exposure to intimate partner violence: Towards a developmental risk and resilience framework for research and intervention. Journal of Family Violence, 22(3), 151-163. doi: 10.1007/s10896-007-9065-3

Gewirtz, A. H., \& Medhanie, A. (2008). Proximity and risk in children's witnessing of intimate partner violence incidents. Journal of Emotional Abuse, 8(1-2), 67-82. doi: 10.1080/10926790801982436

Gilbert, R., Widom, C. S., Browne, K., Fergusson, D., Webb, E., \& Janson, S. (2009). Burden and consequences of child maltreatment in high-income countries. The Lancet, 373(9657), 68-81. doi: 10.1016/S0140-6736(08)61706-7 
International Journal of Child, Youth and Family Studies (2014) 5(4): 493-587

In Harm's Way: A Special Issue on the Impacts and Costs of Witnessing Intimate Partner

Violence

Glueck, S., \& Glueck, E. (1950). Unraveling juvenile delinquency: Exploring the mysteries of human behavior. New York: Commonwealth Fund.

Gordis, E. B., Feres, N., Olezeski, C. L., Rabkin, A. N., \& Trickett, P. K. (2010). Skin conductance reactivity and respiratory sinus arrhythmia among maltreated and comparison youth: Relations with aggressive behavior. Journal of Pediatric Psychology, 35(5), 547-558. doi: 10.1093/jpepsy/jsp113

Gordis, E. B., Margolin, G., Spies, L. A., Susman, E. J., \& Granger, D. A. (2010). Interparental aggression and parent-adolescent salivary alpha amylase symmetry. Physiology \& Behavior, 100(3), 225-233. doi: 10.1016/j.physbeh.2010.01.006

Gover, A. R., Jennings, W. G., Tomsich, E. A., Park, M., \& Rennison, C. M. (2011). The influence of childhood maltreatment and self-control on dating violence: A comparison of college students in the United States and South Korea. Violence and victims, 26(3), 296-318. doi: 10.1891/0886-6708.26.3.296

Graham-Bermann, S. A., Castor, L. E., Miller, L. E., \& Howell, K. H. (2012). The impact of intimate partner violence and additional traumatic events on trauma symptoms and PTSD in preschool-aged children. Journal of Traumatic Stress, 25(4), 393-400. doi: $10.1002 /$ jts. 21724

Graham-Bermann, S. A., Gruber, G., Howell, K. H., \& Girz, L. (2009). Factors discriminating among profiles of resilience and psychopathology in children exposed to intimate partner violence (IPV). Child Abuse \& Neglect, 33(9), 648-660. doi:

10.1016/j.chiabu.2009.01.002

Graham-Bermann, S. A., Howell, K. H., Miller, L. E., Kwek, J., \& Lilly, M. M. (2010). Traumatic events and maternal education as predictors of verbal ability for preschool children exposed to intimate partner violence (IPV). Journal of Family Violence, 25(4), 383-392. doi: 10.1007/s10896-009-9299-3

Graham-Bermann, S. A., \& Perkins, S. (2010). Effects of early exposure and lifetime exposure to intimate partner violence (IPV) on child adjustment. Violence and Victims, 25(4), 427439. doi: 10.1891/0886-6708.25.4.427

Grassi-Oliveira, R., Ashy, M., \& Stein, L. M. (2008). Psychobiology of childhood maltreatment: Effects of allostatic load? Revista Brasileira de Psiquiatria, 30(1), 60-68. doi: 10.1590/S1516-44462008000100012

Gustafsson, H. C., Coffman, J. L., Harris, L. S., Langley, H. A., Ornstein, P. A., \& Cox, M. J. (2013). Intimate partner violence and children's memory. Journal of Family Psychology, 27(6), 937-944. doi: 10.1037/a0034592 
International Journal of Child, Youth and Family Studies (2014) 5(4): 493-587

In Harm's Way: A Special Issue on the Impacts and Costs of Witnessing Intimate Partner

Violence

Haeseler, L. A. (2006). Children of abuse and school discourse: Implications for teachers and administration. Education, 126(3), 534-540. Retrieved from http://www.projectinnovation.biz/education.html

Hall, J. P., \& Parker, K. (2010). Stuck in a loop: Individual and system barriers for job seekers with disabilities. The Career Development Quarterly, 58(3), 246-256. doi: 10.1002/j.2161-0045.2010.tb00190.x

Hamburger, M. E., Leeb, R. T., \& Swahn, M. H. (2008). Childhood maltreatment and early alcohol use among high-risk adolescents. Journal of Studies on Alcohol and Drugs, 69(2), 291-295. Retrieved from http://www.jsad.com

Harding, H. G., Morelen, D., Thomassin, K., Bradbury, L., \& Shaffer, A. (2013). Exposure to maternal-and paternal-perpetrated intimate partner violence, emotion regulation, and child outcomes. Journal of Family Violence, 28(1), 63-72. doi: 10.1007/s10896-0129487-4

Harlow, H. F., Dodsworth, R. O., \& Harlow, M. K. (1965). Total social isolation in monkeys. Proceedings of the National Academy of Sciences of the United States of America, 54(1), 90-97. Retrieved from http://www.pnas.org/

Hasselmann, M. H., \& Reichenheim, M. E. (2006). Parental violence and the occurrence of severe and acute malnutrition in childhood. Paediatric and Perinatal Epidemiology, 20(4), 299-311. doi: 10.1111/j.1365-3016.2006.00735.x

Helweg-Larsen, K., Frederiksen, M. L., \& Larsen, H. B. (2011). Violence, a risk factor for poor mental health in adolescence: A Danish nationally representative youth survey. Scandinavian Journal of Public Health, 39(8), 849-856.

doi: $10.1177 / 1403494811421638$

Herman-Smith, R. (2013). Intimate partner violence exposure in early childhood: An ecobiodevelopmental perspective. Health \& Social Work, 38(4), 231-239. doi: 10.1093/hsw/hlt018

Herrenkohl, T. I., \& Herrenkohl, R. C. (2007). Examining the overlap and prediction of multiple forms of child maltreatment, stressors, and socioeconomic status: A longitudinal analysis of youth outcomes. Journal of Family Violence, 22(7), 553-562. doi: 10.1007/s10896007-9107-x

Herrenkohl, T. I., Sousa, C., Tajima, E. A., Herrenkohl, R. C., \& Moylan, C. A. (2008). Intersection of child abuse and children's exposure to domestic violence. Trauma, Violence, \& Abuse, 9(2), 84-99. doi: 10.1177/1524838008314797 
International Journal of Child, Youth and Family Studies (2014) 5(4): 493-587

In Harm's Way: A Special Issue on the Impacts and Costs of Witnessing Intimate Partner

Violence

Hertzman, C. (2012). Putting the concept of biological embedding in historical perspective. Proceedings of the National Academy of Sciences, 109(Supplement 2), 17160-17167. doi: 10.1073/pnas.1202203109

Hibel, L. C., Granger, D. A., Blair, C., Cox, M. J., \& The Family Life Project Key Investigators. (2009). Intimate partner violence moderates the association between mother-infant adrenocortical activity across an emotional challenge. Journal of Family Psychology, 23(5), 615-625. doi: 10.1037/a0016323

Hibel, L. C., Granger, D. A., Blair, C., Cox, M. J., \& The Family Life Project Key Investigators. (2011). Maternal sensitivity buffers the adrenocortical implications of intimate partner violence exposure during early childhood. Development and Psychopathology, 23(02), 689-701. doi: 10.1017/S0954579411000010

Ho, M. Y., \& Cheung, F. M. (2010). The differential effects of forms and settings of exposure to violence on adolescents' adjustment. Journal of Interpersonal Violence, 25(7), 13091337. doi: 10.1177/0886260509340548

Hoeve, M., Dubas, J. S., Eichelsheim, V. I., van der Laan, P. H., Smeenk, W., \& Gerris, J. R. (2009). The relationship between parenting and delinquency: A meta-analysis. Journal of Abnormal Child Psychology, 37(6), 749-775. doi: 10.1007/s10802-009-9310-8

Holden, G. W. (2003). Children exposed to domestic violence and child abuse: Terminology and taxonomy. Clinical Child and Family Psychology Review, 6(3), 151-160. doi: 10.1023/A:1024906315255

Holmes, M. R. (2013). The sleeper effect of intimate partner violence exposure: Long-term consequences on young children's aggressive behavior. Journal of Child Psychology and Psychiatry, 54(9), 986-995. doi: 10.1111/jcpp.12071

Holt, S., Buckley, H., \& Whelan, S. (2008). The impact of exposure to domestic violence on children and young people: A review of the literature. Child Abuse \& Neglect, 32(8), 797-810. doi: 10.1016/j.chiabu.2008.02.004

Horn, S. R., Hunter, E. C., \& Graham-Bermann, S. A. (2013). Differences and similarities in pairs of siblings exposed to intimate partner violence: A clinical case study. Partner Abuse, 4(2), 274-286. doi: 10.1891/1946-6560.4.2.274

House of Commons Debates. (1982). 32nd Parliament, 1st Session: Vol. 15. Retrieved from http://parl.canadiana.ca/view/oop.debates_HOC3201_15/1?r=0\&s=1.

Hovdestad, W. E., Tonmyr, L., Wekerle, C., \& Thornton, T. (2011). Why is childhood maltreatment associated with adolescent substance abuse? A critical review of explanatory models. International Journal of Mental Health and Addiction, 9(5), 525542. doi: 10.1007/s11469-011-9322-9 
International Journal of Child, Youth and Family Studies (2014) 5(4): 493-587

In Harm's Way: A Special Issue on the Impacts and Costs of Witnessing Intimate Partner

Violence

Howell, K. H. (2011). Resilience and psychopathology in children exposed to family violence. Aggression and Violent Behavior, 16(6), 562-569. doi: 10.1016/j.avb.2011.09.001

Howell, K. H., \& Graham-Bermann, S. A. (2011). The multiple impacts of intimate partner violence on preschool children. In S. A. Graham-Bermann \& A. A. Levendosky (Eds.), How intimate partner violence affects children: Developmental research, case studies, and evidence-based intervention (pp. 87-107). Washington, DC: American Psychological Association.

Howell, K. H., Graham-Bermann, S. A., Czyz, E., \& Lilly, M. (2010). Assessing resilience in preschool children exposed to intimate partner violence. Violence and Victims, 25(2), 150-164. doi: 10.1891/0886-6708.25.2.150

Howells, N. L., \& Rosenbaum, A. (2008). Effects of perpetrator and victim gender on negative outcomes of family violence. Journal of Family Violence, 23(3), 203-209. doi: 10.1007/s10896-007-9144-5

Hungerford, A., Wait, S. K., Fritz, A. M., \& Clements, C. M. (2012). Exposure to intimate partner violence and children's psychological adjustment, cognitive functioning, and social competence: A review. Aggression and Violent Behavior, 17(4), 373-382. doi: 10.1016/j.avb.2012.04.002

Hunter, E. C., \& Graham-Bermann, S. A. (2013). Intimate partner violence and child adjustment: Moderation by father contact? Journal of Family Violence, 28(5), 435-444. doi: 10.1007/s10896-013-9517-x

Huth-Bocks, A. C., Levendosky, A. A., \& Semel, M. A. (2001). The direct and indirect effects of domestic violence on young children's intellectual functioning. Journal of Family Violence, 16(3), 269-290. doi: 10.1023/A:1011138332712

Ireland, T. O., \& Smith, C. A. (2009). Living in partner-violent families: Developmental links to antisocial behavior and relationship violence. Journal of Youth and Adolescence, 38(3), 323-339. doi: 10.1007/s10964-008-9347-y

Israel, E., \& Stover, C. (2009). Intimate partner violence: The role of the relationship between perpetrators and children who witness violence. Journal of Interpersonal Violence, 24(10), 1755-1764. doi: 10.1177/0886260509334044

Jarvis, K. L., Gordon, E. E., \& Novaco, R. W. (2005). Psychological distress of children and mothers in domestic violence emergency shelters. Journal of Family Violence, 20(6), 389-402. doi: 10.1007/s10896-005-7800-1 
International Journal of Child, Youth and Family Studies (2014) 5(4): 493-587

In Harm's Way: A Special Issue on the Impacts and Costs of Witnessing Intimate Partner

Violence

Jayasinghe, S., Jayawardena, P., \& Perera, H. (2009). Influence of intimate partner violence on behaviour, psychological status and school performance of children in Sri Lanka. Journal of Family Studies, 15(3), 274-283. doi: 10.1007/s00127-010-0252-9

Jirapramukpitak, T., Harpham, T., \& Prince, M. (2011). Family violence and its 'adversity package': A community survey of family violence and adverse mental outcomes among young people. Social Psychiatry and Psychiatric Epidemiology, 46(9), 825-831. doi: 10.1007/s00127-010-0252-9

Johannesen, J. K., McGrew, J. H., Griss, M. E., \& Born, D. L. (2009). Change in self-perceived barriers to employment as a predictor of vocational rehabilitation outcome. American Journal of Psychiatric Rehabilitation, 12(4), 295-316. doi: 10.1080/15487760903248358

Johnson, V. K., \& Lieberman, A. F. (2007). Variations in behavior problems of preschoolers exposed to domestic violence: The role of mothers' attunement to children's emotional experiences. Journal of Family Violence, 22(5), 297-308. doi: 10.1007/s10896-0079083-1

Kassis, W., Artz, S., \& Moldenhauer, S. (2013). Laying down the family burden: A crosscultural analysis of resilience in the midst of family violence. Child \& Youth Services, 34(1), 37-63. doi: 10.1080/0145935X.2013.766067

Katz, L. F. (2007). Domestic violence and vagal reactivity to peer provocation. Biological Psychology, 74(2), 154-164. doi: 10.1016/j.biopsycho.2005.10.010

Kaufman, J., Yang, B.-Z., Douglas-Palumberi, H., Crouse-Artus, M., Lipschitz, D., Krystal, J. H., \& Gelernter, J. (2007). Genetic and environmental predictors of early alcohol use. Biological Psychiatry, 61(11), 1228-1234. doi: 10.1016/j.biopsych.2006.06.039

Kemeny, M. E. (2003). The psychobiology of stress. Current Directions in Psychological Science, 12(4), 124-129. doi: 10.1111/1467-8721.01246

Kendler, K. S., Sundquist, K., Ohlsson, H., Palmér, K., Maes, H., Winkleby, M. A., \& Sundquist, J. (2012). Genetic and familial environmental influences on the risk for drug abuse: A national Swedish adoption study. Archives of General Psychiatry, 69(7), 690-697. doi: 10.1001/archgenpsychiatry.2011.2112

Kennedy, A. C., Bybee, D., Sullivan, C. M., \& Greeson, M. (2009). The effects of community and family violence exposure on anxiety trajectories during middle childhood: The role of family social support as a moderator. Journal of Clinical Child \& Adolescent Psychology, 38(3), 365-379. doi: 10.1080/15374410902851713

Kennedy, A. C., Bybee, D., Sullivan, C. M., \& Greeson, M. (2010). The impact of family and community violence on children's depression trajectories: Examining the interactions of 
International Journal of Child, Youth and Family Studies (2014) 5(4): 493-587

In Harm's Way: A Special Issue on the Impacts and Costs of Witnessing Intimate Partner Violence

violence exposure, family social support, and gender. Journal of Family Psychology, 24(2), 197-207. doi: 10.1037/a0018787

Kernic, M. A., Holt, V. L., Wolf, M. E., McKnight, B., Huebner, C. E., \& Rivara, F. P. (2002). Academic and school health issues among children exposed to maternal intimate partner abuse. Archives of Pediatrics \& Adolescent Medicine, 156(6), 549-555. doi: 10.1001/archpedi.156.6.549

Kitzmann, K. M., Gaylord, N. K., Holt, A. R., \& Kenny, E. D. (2003). Child witnesses to domestic violence: A meta-analytic review. Journal of Consulting and Clinical Psychology, 71(2), 339-352. doi: 10.1037/0022-006X.71.2.339

Kliewer, W., \& Murrelle, L. (2007). Risk and protective factors for adolescent substance use: Findings from a study in selected Central American countries. Journal of Adolescent Health, 40(5), 448-455. doi: 10.1016/j.jadohealth.2006.11.148

Knous-Westfall, H. M., Ehrensaft, M. K., MacDonell, K. W., \& Cohen, P. (2012). Parental intimate partner violence, parenting practices, and adolescent peer bullying: A prospective study. Journal of Child and Family Studies, 21(5), 754-766. doi: 10.1007/s10826-011-9528-2

Knutson, J. F., Lawrence, E., Taber, S. M., Bank, L., \& DeGarmo, D. S. (2009). Assessing children's exposure to intimate partner violence. Clinical Child and Family Psychology Review, 12(2), 157-173. doi: 10.1007/s10567-009-0048-1

Kolb, B., Gibb, R., \& Robinson, T. E. (2003). Brain plasticity and behavior. Current Directions in Psychological Science, 12(1), 1-5. doi: 10.1111/1467-8721.01210

Kolbo, J. R., Blakely, E. H., \& Engleman, D. (1996). Children who witness domestic violence: A review of empirical literature. Journal of Interpersonal Violence, 11(2), 281-293. doi: $10.1177 / 088626096011002010$

Koss, K. J., George, M. R., Bergman, K. N., Cummings, E. M., Davies, P. T., \& Cicchetti, D. (2011). Understanding children's emotional processes and behavioral strategies in the context of marital conflict. Journal of Experimental Child Psychology, 109(3), 336-352. doi: 10.1016/j.jecp.2011.02.007

Kuhlman, K. R., Howell, K. H., \& Graham-Bermann, S. A. (2012). Physical health in preschool children exposed to intimate partner violence. Journal of Family Violence, 27(6), 499510. doi: 10.1007/s10896-012-9444-2

Lamers-Winkelman, F., Schipper, J., \& Oosterman, M. (2012). Children’s physical health complaints after exposure to intimate partner violence. British Journal of Health Psychology, 17(4), 771-784. doi: 10.1111/j.2044-8287.2012.02072.x 
International Journal of Child, Youth and Family Studies (2014) 5(4): 493-587

In Harm's Way: A Special Issue on the Impacts and Costs of Witnessing Intimate Partner

Violence

Lamers-Winkelman, F., Willemen, A. M., \& Visser, M. (2012). Adverse childhood experiences of referred children exposed to intimate partner violence: Consequences for their wellbeing. Child Abuse \& Neglect, 36(2), 166-179. doi: 10.1016/j.chiabu.2011.07.006

Lane, K. L., Oakes, W. P., Carter, E. W., Lambert, W. E., \& Jenkins, A. B. (2013). Initial evidence for the reliability and validity of the Student Risk Screening Scale for internalizing and externalizing behaviors at the middle school level. Assessment for Effective Intervention, 39(1), 24-38. doi: 10.1177/1534508413489336

Lang, J. M., \& Stover, C. S. (2008). Symptom patterns among youth exposed to intimate partner violence. Journal of Family Violence, 23(7), 619-629. doi: 10.1007/s10896-008-9184-5

Leeb, R. T., Paulozzi, L. J., Melanson, C., Simon, T. R., \& Arias, I. (2008). Child maltreatment surveillance: Uniform definitions for public health and recommended data elements. Atlanta, GA: Centers for Disease Control and Prevention, National Center for Injury Prevention and Control.

Lepistö, S., Luukkaala, T., \& Paavilainen, E. (2010). Witnessing and experiencing domestic violence: A descriptive study of adolescents. Scandinavian Journal of Caring Sciences, 25(1), 70-80. doi: 10.1111/j.1471-6712.2010.00792.x

Leschied, A., Chiodo, D., Nowicki, E., \& Rodger, S. (2008). Childhood predictors of adult criminality: A meta-analysis drawn from the prospective longitudinal literature. Canadian Journal of Criminology and Criminal Justice/La Revue canadienne de criminologie et de justice pénale, 50(4), 435-467. doi: 10.3138/cjccj.50.4.435

Levendosky, A. A., Bogat, G. A., \& Martinez-Torteya, C. (2013). PTSD symptoms in young children exposed to intimate partner violence. Violence Against Women, 19(2), 187-201. doi: $10.1177 / 1077801213476458$

Liptak, J. (2009). Overcoming barriers to employment success: Instructor's guide. Retrieved from http://ebookbrowse.com/overcoming-barriers-to-employment-success-instructorsguide-pdf-d340778554

Liu, J. (2004). Childhood externalizing behavior: Theory and implications. Journal of Child and Adolescent Psychiatric Nursing, 17(3), 93-103. doi: 10.1111/j.17446171.2004.tb00003.x

Lohman, B. J., Neppl, T. K., Senia, J. M., \& Schofield, T. J. (2013). Understanding adolescent and family influences on intimate partner psychological violence during emerging adulthood and adulthood. Journal of Youth and Adolescence, 42(4), 500-517. doi: 10.1007/s10964-013-9923-7 
International Journal of Child, Youth and Family Studies (2014) 5(4): 493-587

In Harm's Way: A Special Issue on the Impacts and Costs of Witnessing Intimate Partner

Violence

Lundy, M., \& Grossman, S. F. (2005). The mental health and service needs of young children exposed to domestic violence: Supportive data. Families in Society: The Journal of Contemporary Social Services, 86(1), 17-29. doi: 10.1606/1044-3894.1873

Macmillan, R., \& Hagan, J. (2004). Violence in the transition to adulthood: Adolescent victimization, education, and socioeconomic attainment in later life. Journal of Research on Adolescence, 14(2), 127-158. doi: 10.1111/j.1532-7795.2004.01402001.x

Malik, N. M. (2008). Exposure to domestic and community violence in a nonrisk sample associations with child functioning. Journal of Interpersonal Violence, 23(4), 490-504. doi: 10.1177/0886260507312945

Mandal, M., \& Hindin, M. J. (2013). From family to friends: Does witnessing interparental violence affect young adults' relationships with friends? Journal of Adolescent Health, 53(2), 187-193. doi: 10.1016/j.jadohealth.2013.03.015

Manion, I. G. (2010). Provoking evolution in child and youth mental health in Canada. Canadian Psychology/Psychologie canadienne, 51(1), 50-57. doi: 10.1037/a0018468

Margolin, G., \& Gordis, E. B. (2000). The effects of family and community violence on children. Annual Review of Psychology, 51(1), 445-479. doi: 10.1146/annurev.psych.51.1.445

Margolin, G., \& Vickerman, K. A. (2011). Posttraumatic stress in children and adolescents exposed to family violence: I. Overview and issues. Couple and Family Psychology: Research and Practice, 1(S), 63-73. doi: 10.1037/2160-4096.1.S.63

Margolin, G., Vickerman, K. A., Oliver, P. H., \& Gordis, E. B. (2010). Violence exposure in multiple interpersonal domains: Cumulative and differential effects. Journal of Adolescent Health, 47(2), 198-205. doi: 10.1016/j.jadohealth.2010.01.020

Marmion, S. L., \& Lundberg-Love, P. K. (2008). PTSD symptoms in college students exposed to interparental violence: Are they comparable to those that result from child physical and sexual abuse? Journal of Aggression, Maltreatment \& Trauma, 17(3), 263-278. doi: 10.1080/10926770802424935

Martin, S. G. (2002). Children exposed to domestic violence: Psychological considerations for health care practitioners. Holistic Nursing Practice, 16(3), 7-15. Retrieved from http://journals.lww.com/hnpjournal/pages/default.aspx

McCrory, E., De Brito, S. A., \& Viding, E. (2011). The impact of childhood maltreatment: A review of neurobiological and genetic factors. Frontiers in Psychiatry, 2, 1-14. doi: 10.3389/fpsyt.2011.00048 
International Journal of Child, Youth and Family Studies (2014) 5(4): 493-587

In Harm's Way: A Special Issue on the Impacts and Costs of Witnessing Intimate Partner

Violence

McDermott, R., Dawes, C., Prom-Wormley, E., Eaves, L., \& Hatemi, P. K. (2013). MAOA and aggression: A gene-environment interaction in two populations. Journal of Conflict Resolution, 57(6), 1043-1064. doi: 10.1177/0022002712457746

McEwen, B. S. (2000). The neurobiology of stress: From serendipity to clinical relevance. Brain Research, 886(1), 172-189. doi: 10.1016/S0006-8993(00)02950-4

McIntosh, J. E. (2002). Thought in the face of violence: A child's need. Child Abuse \& Neglect, 26(3), 229-241. doi: 10.1016/S0145-2134(01)00321-0

Mejia, R., Kliewer, W., \& Williams, L. (2006). Domestic violence exposure in Colombian adolescents: Pathways to violent and prosocial behavior. Journal of Traumatic Stress, 19(2), 257-267. doi: 10.1002/jts.20116

Meltzer, H., Doos, L., Vostanis, P., Ford, T., \& Goodman, R. (2009). The mental health of children who witness domestic violence. Child \& Family Social Work, 14(4), 491-501. doi: 10.1111/j.1365-2206.2009.00633.x

Mental Health Commission of Canada. (2012). Changing directions, changing lives: The mental health strategy for Canada. Calgary, AB: Author.

Mikolajewski, A. J., Allan, N. P., Hart, S. A., Lonigan, C. J., \& Taylor, J. (2013). Negative affect shares genetic and environmental influences with symptoms of childhood internalizing and externalizing disorders. Journal of Abnormal Child Psychology, 41(3), 411-423. doi: 10.1007/s10802-012-9681-0

Miller, L. E., Howell, K. H., \& Graham-Bermann, S. A. (2012). Potential mediators of adjustment for preschool children exposed to intimate partner violence. Child Abuse \& Neglect, 36(9), 671-675. doi: 10.1016/j.chiabu.2012.07.005

Mitchell, K. J., \& Finkelhor, D. (2001). Risk of crime victimization among youth exposed to domestic violence. Journal of Interpersonal Violence, 16(9), 944-964. doi: 10.1177/088626001016009006

Mohr, W. K., \& Fantuzzo, J. W. (2000). The neglected variable of physiology in domestic violence. Journal of Aggression, Maltreatment \& Trauma, 3(1), 69-84. doi: 10.1300/J146v03n01_06

Mohr, W. K., Lutz, M. J. N., Fantuzzo, J. W., \& Perry, M. A. (2000). Children exposed to family violence: A review of empirical research from a developmental-ecological perspective. Trauma, Violence, \& Abuse, 1(3), 264-283. doi: 10.1177/1524838000001003004

Moore, G. A. (2010). Parent conflict predicts infants' vagal regulation in social interaction. Development and psychopathology, 22(01), 23-33. doi: 10.1017/S095457940999023X 
International Journal of Child, Youth and Family Studies (2014) 5(4): 493-587

In Harm's Way: A Special Issue on the Impacts and Costs of Witnessing Intimate Partner

Violence

Moos, R. H., \& Moos, B. S. (1986). The family environment scale: The manual. Palo Alto, CA: Consulting Psychologists Press.

Moretti, M. M., Obsuth, I., Odgers, C. L., \& Reebye, P. (2006). Exposure to maternal vs. paternal partner violence, PTSD, and aggression in adolescent girls and boys. Aggressive Behavior, 32(4), 385-395. doi: 10.1002/ab.20137

Moss, K. (2004). Kids witnessing family violence. Canadian Social Trends, 73(Summer), 12-16. Retrieved from http://www5.statcan.gc.ca/bsolc/olc-cel/olc-cel?lang=eng\&catno=11-008$\underline{\mathrm{X} 20040016876}$

Moylan, C. A., Herrenkohl, T. I., Sousa, C., Tajima, E. A., Herrenkohl, R. C., \& Russo, M. J. (2010). The effects of child abuse and exposure to domestic violence on adolescent internalizing and externalizing behavior problems. Journal of Family Violence, 25(1), 53-63. doi: 10.1007/s10896-009-9269-9

Murrell, A. R., Christoff, K. A., \& Henning, K. R. (2007). Characteristics of domestic violence offenders: Associations with childhood exposure to violence. Journal of Family Violence, 22(7), 523-532. doi: 10.1007/s10896-007-9100-4

Neighbors, C., Walker, D. D., Mbilinyi, L. F., Zegree, J., Foster, D. W., \& Roffman, R. A. (2013). A self-determination model of childhood exposure, perceived prevalence, justification, and perpetration of intimate partner violence. Journal of Applied Social Psychology, 43(2), 338-349. doi: 10.1111/j.1559-1816.2012.01003.x

Nelson, C. A. (2007). A neurobiological perspective on early human deprivation. Child Development Perspectives, 1(1), 13-18. doi: 10.1111/j.1750-8606.2007.00004.x

Nelson, C. A., \& Bloom, F. E. (1997). Child development and neuroscience. Child Development, 68(5), 970-987. doi: 10.1111/j.1467-8624.1997.tb01974.x

Nguyen, T. D., \& Larsen, S. (2012). Prevalence of children witnessing parental violence. Review of European Studies, 4(1), 148-154. doi: 10.5539/res.v4n1p148

Noll, J. G., \& Shenk, C. E. (2010). Introduction to the special issue: The physical health consequences of childhood maltreatment-Implications for public health. Journal of Pediatric Psychology, 35(5), 447-449. doi: 10.1093/jpepsy/jsq013

Obradović, J., \& Boyce, W. T. (2009). Individual differences in behavioral, physiological, and genetic sensitivities to contexts: Implications for development and adaptation. Developmental Neuroscience, 31(4), 300-308. doi: 10.1159/000216541

Obradović, J., Bush, N. R., \& Boyce, W. T. (2011). The interactive effect of marital conflict and stress reactivity on externalizing and internalizing symptoms: The role of laboratory 
International Journal of Child, Youth and Family Studies (2014) 5(4): 493-587

In Harm's Way: A Special Issue on the Impacts and Costs of Witnessing Intimate Partner

Violence

stressors. Development and Psychopathology, 23(01), 101-114. doi:

10.1017/S0954579410000672

Olaya, B., Ezpeleta, L., de la Osa, N., Granero, R., \& Doménech, J. M. (2010). Mental health needs of children exposed to intimate partner violence seeking help from mental health services. Children and Youth Services Review, 32(7), 1004-1011. doi:

10.1016/j.childyouth.2010.03.028

Olofsson, N., Lindqvist, K., Gådin, K. G., Bråbäck, L., \& Danielsson, I. (2011). Physical and psychological symptoms and learning difficulties in children of women exposed and nonexposed to violence: A population-based study. International Journal of Public Health, 56(1), 89-96. doi: 10.1007/s00038-010-0165-0

Olofsson, N., Lindqvist, K., Shaw, B. A., \& Danielsson, I. (2012). Long-term health consequences of violence exposure in adolescence: A 26-year prospective study. BMC Public Health, 12(1), 411. doi: 10.1186/1471-2458-12-411

Onyskiw, J. E. (2002). Health and use of health services of children exposed to violence in their families. Canadian Journal of Public Health, 93(6), 416-420. Retrieved from http://journal.cpha.ca/index.php/cjph

Owen, A. E., Thompson, M. P., Mitchell, M. D., Kennebrew, S. Y., Paranjape, A., Reddick, T. L., ... Kaslow, N. J. (2008). Perceived social support as a mediator of the link between intimate partner conflict and child adjustment. Journal of Family Violence, 23(4), 221230. doi: 10.1007/s10896-007-9145-4

Owen, A. E., Thompson, M. P., Shaffer, A., Jackson, E. B., \& Kaslow, N. J. (2009). Family variables that mediate the relation between intimate partner violence (IPV) and child adjustment. Journal of Family Violence, 24(7), 433-445. doi:10.1007/s10896-009-9239-2

Paglia-Boak, A., Mann, R. E., Adlaf, E. M., Rehm, J., \& Ontario Student Drug Use. (2009). Drug use among Ontario students: 1997-2011 (CAMH Research Document Series no. 32). Toronto: Centre for Addiction and Mental Health.

Park, A., Smith, C., \& Ireland, T. (2012). Equivalent harm? The relative roles of maltreatment and exposure to intimate partner violence in antisocial outcomes for young adults. Children and Youth Services Review, 34(5), 962-972. doi: 10.1016/j.childyouth.2012.01.029

Peek-Asa, C., Maxwell, L., Stromquist, A., Whitten, P., Limbos, M. A., \& Merchant, J. (2007). Does parental physical violence reduce children's standardized test score performance? Annals of Epidemiology, 17(11), 847-853. doi: 10.1016/j.annepidem.2007.06.004 
International Journal of Child, Youth and Family Studies (2014) 5(4): 493-587

In Harm's Way: A Special Issue on the Impacts and Costs of Witnessing Intimate Partner

Violence

Perry, B. D., \& Pollard, R. (1998). Homeostasis, stress, trauma, and adaptation: A neurodevelopmental view of childhood trauma. Child and Adolescent Psychiatric Clinics of North America, 7(1), 33-51. Retrieved from http://www.childpsych.theclinics.com/

Pierucci-Lagha, A., Gelernter, J., Feinn, R., Cubells, J. F., Pearson, D., Pollastri, A., ... Kranzler, H. R. (2005). Diagnostic reliability of the Semi-structured Assessment for Drug Dependence and Alcoholism (SSADDA). Drug and Alcohol Dependence, 80(3), 303312. doi: 10.1016/j.drugalcdep.2005.04.005

Porter, C. L., Wouden-Miller, M., Silva, S. S., \& Porter, A. E. (2003). Marital harmony and conflict: Links to infants' emotional regulation and cardiac vagal tone. Infancy, 4(2), 297-307. doi: 10.1207/S15327078IN0402_09

Public Health Agency of Canada. (2010). Canadian incidence study of reported child abuse and neglect - 2008: Major findings. Ottawa: Author.

Public Health Agency of Canada. (2013). Family violence initiative (FVI). Ottawa: Author. Retrieved from http://www.phac-aspc.gc.ca/ncfv-cnivf/initiative-eng.php.

Ramiro, L. S., Madrid, B. J., \& Brown, D. W. (2010). Adverse childhood experiences (ACE) and health-risk behaviors among adults in a developing country setting. Child Abuse \& Neglect, 34(11), 842-855. doi: 10.1016/j.chiabu.2010.02.012

Renner, L. M. (2012). Single types of family violence victimization and externalizing behaviors among children and adolescents. Journal of Family Violence, 27(3), 177-186. doi: 10.1007/s10896-012-9421-9

Rhoades, K. A. (2008). Children's responses to interparental conflict: A meta-analysis of their associations with child adjustment. Child Development, 79(6), 1942-1956. doi: 10.1111/j.1467-8624.2008.01235.x

Rico, E., Fenn, B., Abramsky, T., \& Watts, C. (2011). Associations between maternal experiences of intimate partner violence and child nutrition and mortality: Findings from demographic and health surveys in Egypt, Honduras, Kenya, Malawi and Rwanda. Journal of Epidemiology and Community Health, 65(4), 360-367. doi: 10.1136/jech.2008.081810

Rigterink, T., Katz, L. F., \& Hessler, D. M. (2010). Domestic violence and longitudinal associations with children's physiological regulation abilities. Journal of Interpersonal Violence, 25(9), 1669-1683. doi: 10.1177/0886260509354589

Ritter, J., Stewart, M., Bernet, C., Coe, M., \& Brown, S. A. (2002). Effects of childhood exposure to familial alcoholism and family violence on adolescent substance use, conduct problems, and self-esteem. Journal of Traumatic Stress, 15(2), 113-122. doi: 10.1023/A:1014803907234 
International Journal of Child, Youth and Family Studies (2014) 5(4): 493-587

In Harm's Way: A Special Issue on the Impacts and Costs of Witnessing Intimate Partner

Violence

Roberts, A. L., Gilman, S. E., Fitzmaurice, G., Decker, M. R., \& Koenen, K. C. (2010). Witness of intimate partner violence in childhood and perpetration of intimate partner violence in adulthood. Epidemiology, 21(6), 809-818. doi: 10.1097/EDE.0b013e3181f39f03

Rodríguez, M., Valentine, J. M., Son, J. B., \& Muhammad, M. (2009). Intimate partner violence and barriers to mental health care for ethnically diverse populations of women. Trauma, Violence, \& Abuse, 10(4), 358-374. doi: 10.1177/1524838009339756

Rothman, E. F., Edwards, E. M., Heeren, T., \& Hingson, R. W. (2008). Adverse childhood experiences predict earlier age of drinking onset: Results from a representative U.S. sample of current or former drinkers. Pediatrics, 122(2), e298-e304. doi: 10.1542/peds.2007-3412

Roustit, C., Renahy, E., Guernec, G., Lesieur, S., Parizot, I., \& Chauvin, P. (2009). Exposure to interparental violence and psychosocial maladjustment in the adult life course: Advocacy for early prevention. Journal of Epidemiology and Community Health, 63(7), 563-568. doi: 10.1136/jech.2008.077750

Royal Canadian Mounted Police. (2012). The effects of family violence on children - Where does it hurt? Ottawa: Author.

Rutter, M. (2012). Achievements and challenges in the biology of environmental effects. Proceedings of the National Academy of Sciences, 109(Supplement 2), 17149-17153. doi: 10.1073/pnas.1121258109

Saltzman, K. M., Holden, G. W., \& Holahan, C. J. (2005). The psychobiology of children exposed to marital violence. Journal of Clinical Child and Adolescent Psychology, 34(1), 129-139. doi: 10.1207/s15374424jccp3401_12

Sansone, R. A., Leung, J. S., \& Wiederman, M. W. (2012). Five forms of childhood trauma: Relationships with employment in adulthood. Child Abuse \& Neglect, 36(9), 676-679. doi: 10.1016/j.chiabu.2012.07.007

Saxbe, D. E., Margolin, G., Spies Shapiro, L. A., \& Baucom, B. R. (2012). Does dampened physiological reactivity protect youth in aggressive family environments? Child development, 83(3), 821-830. doi: 10.1111/j.1467-8624.2012.01752.x

Scarpa, A., Tanaka, A., \& Chiara Haden, S. (2008). Biosocial bases of reactive and proactive aggression: The roles of community violence exposure and heart rate. Journal of Community Psychology, 36(8), 969-988. doi: 10.1002/jcop.20276

Schensul, J. J., Convey, M., \& Burkholder, G. (2005). Challenges in measuring concurrency, agency and intentionality in polydrug research. Addictive Behaviors, 30(3), 571-574. doi: 10.1016/j.addbeh.2004.05.022 
International Journal of Child, Youth and Family Studies (2014) 5(4): 493-587

In Harm's Way: A Special Issue on the Impacts and Costs of Witnessing Intimate Partner

Violence

Schewe, P., Riger, S., Howard, A., Staggs, S. L., \& Mason, G. E. (2006). Factors associated with domestic violence and sexual assault victimization. Journal of Family Violence, 21(7), 469-475. doi: 10.1007/s10896-006-9042-2

Schwartz, I. M., Rendon, J. A., \& Hsieh, C.-M. (1994). Is child maltreatment a leading cause of delinquency? Child Welfare: Journal of Policy, Practice, and Program, 73(5), 639-655. Retrieved from https://http://www.cwla.org/articles/cwjabstracts.htm

Schwartz, J. P., Hage, S. M., Bush, I., \& Burns, L. K. (2006). Unhealthy parenting and potential mediators as contributing factors to future intimate violence: A review of the literature. Trauma, Violence, \& Abuse, 7(3), 206-221. doi: 10.1177/1524838006288932

Shonkoff, J. P. (2003). From neurons to neighborhoods: Old and new challenges for developmental and behavioral pediatrics. Journal of Developmental \& Behavioral Pediatrics, 24(1), 70-76. doi: 0196-206X/00/2401-0070

Shonkoff, J. P., Boyce, W. T., \& McEwen, B. S. (2009). Neuroscience, molecular biology, and the childhood roots of health disparities: Building a new framework for health promotion and disease prevention. Journal of the American Medical Association, 301(21), 22522259. doi: 10.1001/jama.2009.754

Sigurdsson, S. O., Ring, B. M., O'Reilly, K., \& Silverman, K. (2012). Barriers to employment among unemployed drug users: Age predicts severity. The American Journal of Drug and Alcohol Abuse, 38(6), 580-587. doi: 10.3109/00952990.2011.643976

Silverman, J. G., Decker, M. R., Cheng, D. M., Wirth, K., Saggurti, N., McCauley, H. L., ... Raj, A. (2011). Gender-based disparities in infant and child mortality based on maternal exposure to spousal violence: The heavy burden borne by Indian girls. Archives of Pediatrics \& Adolescent Medicine, 165(1), 22-27. doi: 10.1001/archpediatrics.2010.261

Singh, V.-A. S., Thornton, T., \& Tonmyr, L. (2011). Determinants of substance abuse in a population of children and adolescents involved with the child welfare system. International Journal of Mental Health and Addiction, 9(4), 382-397. doi: 10.1007/s11469-011-9320-y

Sinha, M. (2013). Family violence in Canada: A statistical profile, 2011. Ottawa: Canadian Centre for Justice Statistics.

Skeer, M., McCormick, M. C., Normand, S.-L. T., Buka, S. L., \& Gilman, S. E. (2009). A prospective study of familial conflict, psychological stress, and the development of substance use disorders in adolescence. Drug and Alcohol Dependence, 104(1), 65-72. doi: 10.1016/j.drugalcdep.2009.03.017 
International Journal of Child, Youth and Family Studies (2014) 5(4): 493-587

In Harm's Way: A Special Issue on the Impacts and Costs of Witnessing Intimate Partner

Violence

Slade, T. (2007). The descriptive epidemiology of internalizing and externalizing psychiatric dimensions. Social Psychiatry and Psychiatric Epidemiology, 42(7), 554-560. doi: 10.1007/s00127-007-0200-5

Slopen, N., Fitzmaurice, G. M., Williams, D. R., \& Gilman, S. E. (2012). Common patterns of violence experiences and depression and anxiety among adolescents. Social Psychiatry and Psychiatric Epidemiology, 47(10), 1591-1605. doi: 10.1007/s00127-011-0466-5

Smith, C. A., Elwyn, L. J., Ireland, T. O., \& Thornberry, T. P. (2010). Impact of adolescent exposure to intimate partner violence on substance use in early adulthood. Journal of Studies on Alcohol and Drugs, 71(2), 219-230. Retrieved from http://www.jsad.com/

Sobkoviak, R. M., Yount, K. M., \& Halim, N. (2012). Domestic violence and child nutrition in Liberia. Social Science \& Medicine, 74(2), 103-111. doi:

10.1016/j.socscimed.2011.10.024

Sousa, C., Herrenkohl, T. I., Moylan, C. A., Tajima, E. A., Klika, J. B., Herrenkohl, R. C., \& Russo, M. J. (2011). Longitudinal study on the effects of child abuse and children's exposure to domestic violence, parent-child attachments, and antisocial behavior in adolescence. Journal of Interpersonal Violence, 26(1), 111-136. doi: $0.1177 / 0886260510362883$

Spilsbury, J. C., Belliston, L., Drotar, D., Drinkard, A., Kretschmar, J., Creeden, R., ... Friedman, S. (2007). Clinically significant trauma symptoms and behavioral problems in a community-based sample of children exposed to domestic violence. Journal of Family Violence, 22(6), 487-499. doi: 10.1007/s10896-007-9113-z

Spilsbury, J. C., Kahana, S., Drotar, D., Creeden, R., Flannery, D. J., \& Friedman, S. (2008). Profiles of behavioral problems in children who witness domestic violence. Violence and Victims, 23(1), 3-17. doi: 10.1891/0886-6708.23.1.3

Steinberg, L., \& Avenevoli, S. (2000). The role of context in the development of psychopathology: A conceptual framework and some speculative propositions. Child Development, 71(1), 66-74. doi: 10.1111/1467-8624.00119

Sternberg, K. J., Baradaran, L. P., Abbott, C. B., Lamb, M. E., \& Guterman, E. (2006). Type of violence, age, and gender differences in the effects of family violence on children's behavior problems: A mega-analysis. Developmental Review, 26(1), 89-112. doi: 10.1016/j.dr.2005.12.001

Sternberg, K. J., Lamb, M. E., Guterman, E., \& Abbott, C. B. (2006). Effects of early and later family violence on children's behavior problems and depression: A longitudinal, multiinformant perspective. Child Abuse \& Neglect, 30(3), 283-306. doi:

10.1016/j.chiabu.2005.10.008 
International Journal of Child, Youth and Family Studies (2014) 5(4): 493-587

In Harm's Way: A Special Issue on the Impacts and Costs of Witnessing Intimate Partner

Violence

Straus, M. A. (1979). Measuring intrafamily conflict and violence: The conflict tactics (CT) scales. Journal of Marriage and the Family, 41(1), 75-88. Retrieved from http://onlinelibrary.wiley.com/journal/10.1111/(ISSN)1741-3737

Straus, M. A., Hamby, S. L., Boney-McCoy, S., \& Sugarman, D. B. (1996). The revised conflict tactics scales (CTS2): Development and preliminary psychometric data. Journal of Family Issues, 17(3), 283-316. doi: 10.1177/019251396017003001

Stride, S., Geffner, R., \& Lincoln, A. (2008). The physiological and traumatic effects of childhood exposure to intimate partner violence. Journal of Emotional Abuse, 8(1-2), 83101. doi: 10.1080/10926790801984507

Strine, T. W., Dube, S. R., Edwards, V. J., Prehn, A. W., Rasmussen, S., Wagenfeld, M., ... Croft, J. B. (2012). Associations between adverse childhood experiences, psychological distress, and adult alcohol problems. American Journal of Health Behavior, 36(3), 408423. doi: 10.5993/AJHB.36.3.11

Sturge-Apple, M. L., Davies, P. T., Cicchetti, D., \& Manning, L. G. (2012). Interparental violence, maternal emotional unavailability and children's cortisol functioning in family contexts. Developmental Psychology, 48(1), 237-249. doi: 10.1037/a0025419

Subramanian, S., Ackerson, L. K., Subramanyam, M. A., \& Wright, R. J. (2007). Domestic violence is associated with adult and childhood asthma prevalence in India. International Journal of Epidemiology, 36(3), 569-579. doi: 10.1093/ije/dym007

Suglia, S. F., Enlow, M. B., Kullowatz, A., \& Wright, R. J. (2009). Maternal intimate partner violence and increased asthma incidence in children: Buffering effects of supportive caregiving. Archives of Pediatrics \& Adolescent Medicine, 163(3), 244-250. doi: 10.1001/archpediatrics.2008.555

Tajima, E. A., Herrenkohl, T. I., Moylan, C. A., \& Derr, A. S. (2011). Moderating the effects of childhood exposure to intimate partner violence: The roles of parenting characteristics and adolescent peer support. Journal of Research on Adolescence, 21(2), 376-394. doi: 10.1111/j.1532-7795.2010.00676.x

Tam, T. W., Zlotnick, C., \& Robertson, M. J. (2003). Longitudinal perspective: Adverse childhood events, substance use, and labor force participation among homeless adults. The American Journal of Drug and Alcohol Abuse, 29(4), 829-846. doi: 10.1081/ADA120026263

Tanaka, M., Jamieson, E., Georgiades, K., Duku, E. K., Boyle, M. H., \& MacMillan, H. L. (2011). The association between childhood abuse and labor force outcomes in young adults: Results from the Ontario child health study. Journal of Aggression, Maltreatment \& Trauma, 20(8), 821-844. doi: 10.1080/10926771.2011.621851 
International Journal of Child, Youth and Family Studies (2014) 5(4): 493-587

In Harm's Way: A Special Issue on the Impacts and Costs of Witnessing Intimate Partner

Violence

Teicher, M. H. (2000). Wounds that time won't heal: The neurobiology of child abuse. Cerebrum, 2(4), 50-67. Retrieved from http://192.211.16.13/curricular/hhd2006/news/wounds.pdf

Teicher, M. H., Samson, J., Polcari, A., \& McGreenery, C. (2006). Sticks, stones, and hurtful words: Relative effects of various forms of childhood maltreatment. American Journal of Psychiatry, 163(6), 993-1000. doi: 10.1176/appi.ajp.163.6.993

Teicher, M. H., Samson, J. A., Sheu, Y.-S., Polcari, A., \& McGreenery, C. E. (2010). Hurtful words: Association of exposure to peer verbal abuse with elevated psychiatric symptom scores and corpus callosum abnormalities. American Journal of Psychiatry, 167(12), 1464-1471. doi: 10.1176/appi.ajp.2010.10010030

Teicher, M. H., Tomoda, A., \& Andersen, S. L. (2006). Neurobiological consequences of early stress and childhood maltreatment: Are results from human and animal studies comparable? Annals of the New York Academy of Sciences, 1071(1), 313-323. doi: 10.1196/annals.1364.024

Teicher, M. H., \& Vitaliano, G. D. (2011). Witnessing violence toward siblings: An understudied but potent form of early adversity. PLOS ONE, 6(12), 1-10. doi: 10.1371/journal.pone.0028852

Temple, J. R., Shorey, R. C., Tortolero, S. R., Wolfe, D. A., \& Stuart, G. L. (2013). Importance of gender and attitudes about violence in the relationship between exposure to interparental violence and the perpetration of teen dating violence. Child Abuse \& Neglect, 37(5), 343-352. doi: 10.1016/j.chiabu.2013.02.001

Thompson, R., \& Whimper, L. A. (2010). Exposure to family violence and reading level of early adolescents. Journal of Aggression, Maltreatment \& Trauma, 19(7), 721-733. doi: $10.1080 / 10926771003781347$

Thompson, R., Wiley, T. R., Lewis, T., English, D. J., Dubowitz, H., Litrownik, A. J., ... Block, S. (2012). Links between traumatic experiences and expectations about the future in high risk youth. Psychological Trauma: Theory, Research, Practice, and Policy, 4(3), 293302. doi: 10.1037/a0023867

Thornberry, T. P., Krohn, M. D., Lizotte, A. J., Smith, C. A., \& Porter, P. (1998). Taking stock: An overview of findings from the Rochester Youth Development Study. Paper presented at the American Society of Criminology meeting, Washington, DC. Arbuckle, Frabutt, MacKinnon-Lewis.

Towe-Goodman, N. R., Stifter, C. A., Mills-Koonce, W. R., Granger, D. A., \& The Family Life Project Key Investigators. (2012). Interparental aggression and infant patterns of adrenocortical and behavioral stress responses. Developmental psychobiology, 54(7), 685-699. doi: 10.1002/dev.20618 
International Journal of Child, Youth and Family Studies (2014) 5(4): 493-587

In Harm's Way: A Special Issue on the Impacts and Costs of Witnessing Intimate Partner

Violence

Tschann, J. M., Pasch, L. A., Flores, E., Marin, B. V., Baisch, E. M., \& Wibbelsman, C. J. (2009). Nonviolent aspects of interparental conflict and dating violence among adolescents. Journal of Family Issues, 30(3), 295-319. doi: 10.1177/0192513X08325010

Turner, H. A., Finkelhor, D., \& Ormrod, R. (2006). The effect of lifetime victimization on the mental health of children and adolescents. Social Science \& Medicine, 62(1), 13-27. doi: 10.1016/j.socscimed.2005.05.030

Turpel-Lafond, M. E. (2012). Honouring Kaitlynne, Max and Cordon: Make their voices heard now. Victoria, BC: BC Representative for Children and Youth.

U.S. Department of Justice Office of Juvenile Justice and Delinquency Prevention. (1998). Consequences of youth substance abuse. Washington, DC: Author.

Varcoe, C., Hankivsky, O., Ford-Gilboe, M., Wuest, J., Wilk, P., Hammerton, J., \& Campbell, J. C. (2011). Attributing selected costs to intimate partner violence in a sample of women who have left abusive partners: A social determinants of health approach. Canadian Public Policy, 37(3), 359-380.

Voisin, D. R., \& Hong, J. S. (2012). A meditational model linking witnessing intimate partner violence and bullying behaviors and victimization among youth. Educational Psychology Review, 24(4), 479-498. doi: 10.1007/s10648-012-9197-8

Widom, C. S., Marmorstein, N. R., \& White, H. R. (2006). Childhood victimization and illicit drug use in middle adulthood. Psychology of Addictive Behaviors, 20(4), 394-403.

Wolfe, D. A., Crooks, C. V., Lee, V., McIntyre-Smith, A., \& Jaffe, P. G. (2003). The effects of children's exposure to domestic violence: A meta-analysis and critique. Clinical Child and Family Psychology Review, 6(3), 171-187. doi: 10.1023/A:1024910416164

Wood, S. L., \& Sommers, M. S. (2011). Consequences of intimate partner violence on child witnesses: A systematic review of the literature. Journal of Child and Adolescent Psychiatric Nursing, 24(4), 223-236. doi: 10.1111/j.1744-6171.2011.00302.x

World Health Organization [WHO]. (2013). The Ottawa charter for health promotion. Retrieved from http://www.who.int/healthpromotion/conferences/previous/ottawa/en/

Wright, E. M., \& Fagan, A. A. (2012). Exposure to intimate partner violence. Does the gender of the perpetrator matter for adolescent mental health outcomes? Criminal Justice and Behavior, 39(1), 26-41. doi: 10.1177/0093854811425649

Ybarra, G. J., Wilkens, S. L., \& Lieberman, A. F. (2007). The influence of domestic violence on preschooler behavior and functioning. Journal of Family Violence, 22(1), 33-42. doi: 10.1007/s10896-006-9054-y 
International Journal of Child, Youth and Family Studies (2014) 5(4): 493-587

In Harm's Way: A Special Issue on the Impacts and Costs of Witnessing Intimate Partner

Violence

Yount, K. M., DiGirolamo, A. M., \& Ramakrishnan, U. (2011). Impacts of domestic violence on child growth and nutrition: A conceptual review of the pathways of influence. Social Science \& Medicine, 72(9), 1534-1554. doi: 10.1016/j.socscimed.2011.02.042

Zahn, M. A. (2009). The delinquent girl. Philedelphia: Temple University Press.

Zarling, A. L., Taber-Thomas, S., Murray, A., Knuston, J. F., Lawrence, E., Valles, N.-L., ... Bank, L. (2013). Internalizing and externalizing symptoms in young children exposed to intimate partner violence: Examining intervening processes. Journal of Family Psychology, 27(6), 945-955. doi: 10.1037/a0034804

Zielinski, D. S. (2009). Child maltreatment and adult socioeconomic well-being. Child Abuse \& Neglect, 33(10), 666-678. doi: 10.1016/j.chiabu.2009.09.001

Zinzow, H. M., Ruggiero, K. J., Hanson, R. F., Smith, D. W., Saunders, B. E., \& Kilpatrick, D. G. (2009). Witnessed community and parental violence in relation to substance use and delinquency in a national sample of adolescents. Journal of Traumatic Stress, 22(6), 525533. doi: $10.1002 /$ jts.20469 